JOURNAL OF THE

AMERICAN MATHEMATICAL SOCIETY

Volume 11, Number 2, April 1998, Pages 321-361

S 0894-0347(98)00256-2

\title{
QUASI-ISOMETRIC RIGIDITY OF NONUNIFORM LATTICES IN HIGHER RANK SYMMETRIC SPACES
}

\author{
ALEX ESKIN
}

We recall the following:

Definition. Let $X$ and $Y$ be metric spaces. A $(\kappa, C)$ quasi-isometric embedding is a map $\phi: X \longrightarrow Y$, such that

$$
\frac{1}{\kappa} d_{X}\left(x_{1}, x_{2}\right)-C \leq d_{Y}\left(\phi\left(x_{1}\right), \phi\left(x_{2}\right)\right) \leq \kappa d_{X}\left(x_{1}, x_{2}\right)+C .
$$

Thus $\phi$ is bi-Lipschitz on large scales.

If there exists a constant $C^{\prime}$ such that every point of $Y$ is within a distance $C^{\prime}$ of a point of $\phi(X)$, then there is a quasi-isometric embedding $\psi: Y \rightarrow X$ which is a "coarse inverse of $\phi "$, i.e. $\sup _{x \in X} d_{X}(x, \psi(\phi(x)))<\infty$ and $\sup _{y \in Y} d_{Y}(y, \phi(\psi(y)))<$ $\infty$. In this case $\phi$ is called a quasi-isometry and the spaces $X$ and $Y$ are said to be quasi-isometric.

A basic example to keep in mind is that the fundamental group $\pi_{1}(M)$ (endowed with the word metric) of a compact Riemannian manifold $M$ is quasi-isometric to the universal cover $\widetilde{M}$ of $M$.

In this paper we prove the following theorem:

Theorem 0.1. Let $\Gamma$ be an irreducible nonuniform lattice in a semisimple Lie group $G$ with finite center and no rank 1 factors. Let $\phi$ be a quasi-isometry of $\Gamma$ to itself, in the word metric. Then there exists an element $g$ in the commensurator of $\Gamma$ so that $\phi$ is a bounded distance away from left multiplication by $g$. In particular, $Q I(\Gamma) \cong \operatorname{Comm}(\Gamma)$.

Corollary 0.2. Suppose $\Gamma$ is an irreducible nonuniform lattice in a semisimple Lie group $G$ with finite center and no rank 1 factors, and suppose $\Lambda$ is a finitely generated group quasi-isometric to $\Gamma$. Then there exists a short exact sequence

$$
1 \rightarrow F \rightarrow \Lambda \rightarrow \Gamma^{\prime} \rightarrow 1
$$

where $F$ is a finite group, and $\Gamma^{\prime}$ is a lattice commensurable to $\Gamma$ up to conjugation. In particular, two nonuniform irreducible lattices in $G$ are quasi-isometric if and only if they are commensurable up to conjugation.

We note that the rigidity result for nonuniform lattices is much stronger than the analogous result for uniform lattices (in the higher rank case the result for uniform lattices was first proved in $[\mathrm{KL}]$; see also [EF] for another proof). This phenomenon

Received by the editors October 28, 1996 and, in revised form, October 21, 1997.

1991 Mathematics Subject Classification. Primary 22E40, 20 F32.

Key words and phrases. Lie groups, discrete subgroups, geometric group theory.

The author was supported in part by an N.S.F. Postdoctoral Fellowship.

(C)1998 American Mathematical Society 
was first noticed by Richard Schwartz who did much of the work on the subject, and conjectured Theorem 0.1 and Corollary 0.2 in full generality.

In [S1] Theorem 0.1 and Corollary 0.2 were proved using geometric methods in the case where the rank of $G$ is 1 , so that the symmetric space has negative curvature (the case $G=S L(2, \mathbb{R})$ has to be excluded). In [FS] the same result was proved for the case of quadratic Hilbert modular groups, and it was extended to arbitrary Hilbert modular groups in [S2]. The passage to higher rank and nonpositive curvature required development of new methods, in particular the "coarse topology" and "coarse separation theorem" of [FS] and [S2]. Even though these methods are quite general, and have proved to be useful in many other questions of coarse geometry (for example $[\mathrm{FM}]$ ), they do not seem to be suitable for the case when the $\mathbb{Q}$-rank of the lattice is bigger than 1 , e.g. $S L(3, \mathbb{Z})$. Our proof is in fact more geometric than topological, and follows a different scheme from the earlier results.

The basic ingredients in our proof are the "quasi-flats with holes" result of [EF], the theorem of Lubotzky-Mozes-Raghunathan establishing the equivalence of the word metric with the induced metric, the Moore ergodicity theorem, and Tits' theory of buildings. The idea of the proof is to prove Theorem 0.1 without extending the map $\phi$ to the entire symmetric space. Instead (working in the induced metric), we use the quasi-flats with holes result and the Moore ergodicity theorem to construct a map $\phi_{0}$ of the boundary defined almost everywhere. We then show that this map is bi-Hölder on its domain of definition. This allows us to extend $\phi_{0}$ to a homeomorphism of the boundaries. We then show that this extended map induces an order preserving bijection of the associated Tits buildings, which implies by Tits' theorem that $\phi_{0}$ is induced by an isometry. Then we show that $\phi$ is within a bounded distance of that isometry. A final argument shows that the element $g \in G$ inducing the isometry belongs in fact to the commensurator of $\Gamma$.

For an alternative approach to parts of the argument see [Dru].

Most of the argument (through and including the proof of Proposition 6.1) does not use the assumption that $G$ has no rank one factors but only the weaker assumption $\operatorname{rank} G \geq 2$. In the case when $\operatorname{rank} G \geq 2$ but $G$ contains rank one factors, we write down an intermediate result, Proposition 10.1, which follows naturally from the argument in this paper. Proposition 10.1 can then be combined with results from [FS], [S1] and [S2] to prove the analogues of Theorem 0.1 and Corollary 0.2 for this case; see $[\mathrm{Fa}]$ for an outline of the argument.

\section{Preliminaries}

Notational conventions. We denote by $\mu(\cdot)$ the Haar measure on $\Gamma \backslash G$, normalized so that $\mu(\Gamma \backslash G)=1$. The Euclidean measure on $\mathbb{R}^{n}$ is denoted by $\nu$ or $|\cdot|$. We denote the measure of the unit ball in $\mathbb{R}^{n}$ by $\omega_{n}$. The complement of a set $U$ is denoted $U^{c}$.

Let $\pi$ denote the natural projection from $G$ to the symmetric space $G / K=X$, and let $p$ denote the natural projection from $G$ to $\Gamma \backslash G$. The natural projection from $X$ to $\Gamma \backslash X$ is denoted by $\bar{p}$. Let $\bar{\mu}$ denote the measure on $\Gamma \backslash X$ which is the push-forward of $\mu$; then $\bar{\mu}(\Gamma \backslash X)=1$.

We denote the identity element of $G$ by 1 , and $\pi(1) \in X$ by $e$.

1.1. Theorems used in the proof. The proof of Theorem 0.1 is based on the following results: 
Theorem 1.1 (Lubotzky-Mozes-Raghunathan). Let $\Gamma$ be an irreducible lattice in a semisimple Lie group $G$, with $\operatorname{rank} G \geq 2$. Then the word metric on $\Gamma$ is equivalent to the metric induced from the embedding of $\Gamma$ in the symmetric space $G / K$.

Proof. See [LMR].

Theorem 1.2 (Moore ergodicity). Let $\Gamma$ be an irreducible lattice in a semisimple Lie group $G$ with finite center, $H \subset G$ a noncompact subgroup. Then the $H$-action on $\Gamma \backslash G$ is ergodic.

Proof. See [Zim, Theorem 2.2.6].

Theorem 1.3 (Birkhoff ergodic theorem for $\mathbb{R}^{n}$-action). Let $(Y, m)$ be a finite measure space, and suppose $A \cong \mathbb{R}^{n}$ acts on $Y$, preserving $m$. If the action is ergodic, then for any $f \in L^{1}(Y)$ and almost all $y \in Y$,

$$
\lim _{r \rightarrow \infty} \frac{1}{\omega_{n} r^{n}} \int_{B_{r}} f(a \cdot y) d \nu(a)=\int_{Y} f d m
$$

where $B_{r}=B(0, r)$ is the ball of radius $r$ centered at 0 .

Maximal flats and Cartan subgroups. The symmetric space $X$ is a quotient $G / K$, where $G$ is the isometry group of $X$ and $K$ is a maximal compact subgroup. The Killing form $\langle\cdot, \cdot\rangle$ is a nondegenerate quadratic form on the Lie algebra $\mathfrak{g}$ of $G$ defined by $\langle X, Y\rangle=\operatorname{trace}(\operatorname{ad} X \operatorname{ad} Y)$. The Lie algebra $\mathfrak{g}$ can be decomposed as $\mathfrak{k} \oplus \mathfrak{p}$ where $\mathfrak{k}$ is the Lie algebra of $K$ and $\mathfrak{p}$ is the orthogonal complement relative to the Killing form. We fix a maximal Abelian subalgebra $\mathfrak{a}$ in $\mathfrak{p}$, and denote exp $\mathfrak{a}$ by $A$. The group $A$ is Abelian and is called a Cartan subgroup of $G ; \mathfrak{a}$ is a Cartan subalgebra. The dimension of $\mathfrak{a}$ is the rank of $X$ (or of $G$ ), which we denote by $n$. For any $g \in G, \pi(g A) \subset X$ is a maximal flat subspace of $X$; also every maximal flat can be written as $\pi(g A)$ for some $g \in G$.

As an immediate corollary of Theorem 1.2 and Theorem 1.3 one has the following:

Theorem 1.4 (Birkhoff ergodic theorem for Cartan flow). Let $G, \mathfrak{a}$ be as above. Then, for any $f \in L^{1}(\Gamma \backslash G)$ and for $\mu$-a.e. $x \in \Gamma \backslash G$,

$$
\lim _{r \rightarrow \infty} \frac{1}{|B(0, r)|} \int_{B(0, r) \subset \mathfrak{a}} f(x \exp v) d \nu(v)=\int_{\Gamma \backslash G} f d \mu .
$$

Swiss cheese. Let $U$ be a subset of $\mathbb{R}^{n}$. We say that $U$ is "an infinite swiss cheese" if it contains a fraction of the volume, i.e. there exists $\delta<1$ so that for every $x \in U$,

$$
\liminf _{r \rightarrow \infty} \frac{|U \cap B(x, r)|}{|B(x, r)|} \geq 1-\delta .
$$

We note that if the complement of $U$ (i.e. the "holes") contains balls of arbitrarily large radius, the bound in (2) cannot be uniform in the choice of $x \in U$. Given $\epsilon>0$ and $R>0$, let

$$
U_{(\epsilon, R)}=\{x \in U: \text { for all } r>R,|U \cap B(x, r)| \geq(1-\epsilon)|B(x, r)|\} .
$$

Hence, $U_{(\epsilon, R)}$ is a subset of $U$ on which a uniform bound exists.

Theorem 1.5 (Quasi-flats with holes). Suppose $\phi$ is a $(\kappa, C)$ quasi-isometric embedding from $U \subset \mathbb{R}^{n}$ to $X$, where $U$ has the induced Euclidean metric. Let $\epsilon>0$ and $R>0$ be constants, with $\epsilon$ sufficiently small (depending only on $\kappa$ ), and $U_{(\epsilon, R)}$ nonempty. Then $\phi\left(U_{(\epsilon, R)}\right)$ lies in the $N$-neighborhood of the union of at most $M$ flats in $X$; here $M=M(\kappa)$ and $N=N(\kappa, \epsilon, R, C)$. 
Proof. See [EF, Theorem 8.1].

1.2. Symmetric spaces and quasi-isometries. Most of this subsection is standard: we review the results mostly to fix notation. In what follows, we follow the notation of $[\mathrm{EF}]$ where possible. Throughout, by a flat we mean a maximal flat.

Weyl chambers, the Weyl group and the longest element. We denote the root system associated to $(G, A)$ by $\Sigma$, and fix a positive system $\Sigma^{+}$and associated simple system $\Delta$ for $\Sigma$. Let $\mathfrak{a}^{+}$denote the subset $\{H \in \mathfrak{a}: \alpha(H)>0$ for all $\alpha \in$ $\Delta\}$; the set $\mathfrak{a}^{+}$is called the positive Weyl chamber. Let $A^{+}=\exp \mathfrak{a}^{+} \subset A$. For any $g \in G$, the orbit $g A^{+}$is a Weyl chamber in $G$; the set $\pi\left(g A^{+}\right)$is a Weyl chamber in $X$.

Let $M$ denote the centralizer of $A$ in $K$. The Weyl group $W$ is the quotient of the normalizer in $K$ of $A$ by $M$. This is a finite group which acts transitively on the Weyl chambers of $A$. Let $w_{0} \in W$ denote the longest element; it takes $\mathfrak{a}^{+}$into $-\mathfrak{a}^{+}$.

Subflats, hyperplanes, polyhedra and orbits of subgroups of $A$. For a subset $\sigma$ of $\Sigma$, let

$$
A_{\sigma}=\{a \in A \mid \alpha(\log a)=0, \quad \forall \alpha \in \sigma\}
$$

If $\sigma \subset \Delta$, let

$$
A_{\sigma}^{+}=\{a \in A: \alpha(\log a)=0, \quad \forall \alpha \in \sigma, \quad \text { and } \alpha(\log a)>0, \quad \forall \alpha \in \Delta-\sigma\} .
$$

We call the orbits of $A_{\sigma}$ " $\sigma$-subflats in $G$ " (or just subflats) and the orbits of $A_{\sigma}^{+}$ " $\sigma$-walls" (or just walls) in $G$. If $\sigma=\emptyset$, then the orbit of $A_{\emptyset}^{+}$(denoted $A^{+}$) is a Weyl chamber in $G$; if $\sigma=\{\alpha\}$ we write $A_{\alpha}$ instead of $A_{\{\alpha\}}$ and call the orbit a hyperplane in $G$. By a subflat, wall, Weyl chamber, or hyperplane in $X$ we mean the projection to $X$ of the corresponding object in $G$ via $\pi$.

It is easy to check that the intersection of two flats is either empty, or a point or a subflat. Conversely, for every subflat $S \subset A$ passing through the identity 1 there exists $k_{S} \in K$ such that $k_{S} A k_{S}^{-1} \cap A=S$.

By a polyhedron we mean a (possibly unbounded) convex subset of a flat bounded by hyperplanes. A flat is also considered a polyhedron. If $U$ is a polyhedron in $X$, we denote by $\partial U$ its boundary as a subset of $\mathbb{R}^{n}$, not as a subset of $X$ (otherwise we will always have $\partial U=U$ ).

The Furstenberg boundary $\hat{X}$ and the Tits building $\mathcal{T}(X)$. The Furstenberg boundary $\hat{X}$ is defined as the equivalence classes of Weyl chambers in $X$, with two chambers considered equivalent if the Hausdorff distance between them is finite. Each Weyl chamber is equivalent to a Weyl chamber with origin at the point $e$. Since every Weyl chamber with origin at $e$ is of the form $\pi\left(k A^{+}\right)$for some $k \in K$, this identifies $\hat{X}$ with $K / M$.

If $\sigma \subset \Delta$, we denote by $P_{\sigma}$ the standard parabolic subgroup associated to $\sigma$. We have the Langlands decomposition $P_{\sigma}=M_{\sigma} A_{\sigma} N_{\sigma}$, where $A_{\sigma}$ is as above, $M_{\sigma}$ is semisimple and $N_{\sigma}$ is unipotent. Write $K_{\sigma}=M_{\sigma} \cap K$. $P_{\emptyset}$ is a Borel subgroup of $G$, denoted by $B$.

Any parabolic subgroup is of the form $g P_{\sigma} g^{-1}$ for some $\sigma \subset \Sigma$ and some $g \in G$. The Tits building $\mathcal{T}(X)$ is the set of parabolic subgroups of $G$, partially ordered by reverse inclusion. Hence the maximal dimension simplices of $\mathcal{T}(X)$ correspond to conjugates of $B$. Since $B$ is its own normalizer, the set of conjugates of $B$ 
is $G / B \cong K / M$, where the last identification used the Iwasawa decomposition $G=K B$, and the fact that $M=K \cap B$.

Thus every maximal dimension simplex of $\mathcal{T}(X)$ corresponds to a point in $K / M$. The same argument shows that every simplex $D$ in $\mathcal{T}(X)$ corresponds to a point in $K / K_{\sigma}$, with $\operatorname{dim}(D)=n-|\sigma|$.

This description of $\mathcal{T}(X)$ is convenient for the argument in this paper. As in [EF] we denote the $K$-invariant distance on $K / M$ by $d_{K}$.

Galleries, combinatorial distance and the singular subset. Each pair of $n$-simplices in the Tits building lies in an apartment. A gallery is a sequence of $n$-simplices, with successive simplices sharing a codimension 1 face. By the Tits combinatorial distance $d_{c}\left(k_{1}, k_{2}\right)$ we mean the length of a minimal gallery in the Tits building connecting the $n$-simplices $k_{1} M$ and $k_{2} M$. This implies that $\sup _{k_{1}, k_{2}} d_{c}\left(k_{1}, k_{2}\right)<\infty$. Let $\ell=\max _{k_{1}, k_{2}} d_{c}\left(k_{1}, k_{2}\right)$, and as in [EF], let $\mathcal{S}=$ $\left\{k \in K \quad: \quad d_{c}(1, k)<\ell\right\}$. The singular subset $\mathcal{S} \subset K$ is closed, and $\mathcal{S}=$ $K \cap \bigcup_{w \in W-\left\{w_{0}\right\}} B w B$, where $B$ is the Borel subgroup. This exhibits $\mathcal{S}$ as a union of cells, of varying dimensions.

Graded quasi-isometric embeddings. If $X_{1}$ and $X_{2}$ are metric spaces, and $z_{0} \in X_{1}$, we say that a map $q: X_{1} \rightarrow X_{2}$ is a $(\kappa, \epsilon, \rho)$ graded quasi-isometric embedding centered at $z_{0}$ if for all $x, y \in X_{1}$ with $d\left(y, z_{0}\right) \geq d\left(x, z_{0}\right)$,

$$
\frac{1}{\kappa} d(x, y)-\max \left(\rho, \epsilon d\left(x, z_{0}\right)\right) \leq d(q(x), q(y)) \leq \kappa d(x, y)+\max \left(\rho, \epsilon d\left(x, z_{0}\right)\right) .
$$

Thus any $(\kappa, \rho)$ quasi-isometric embedding is also a $(\kappa, \epsilon, \rho)$ graded quasi-isometric embedding. Graded quasi-isometric embeddings are more general in the sense that the "coarseness constant" is allowed to grow linearly with distance to the origin $z_{0}$.

Graded q.i. embeddings (cf. [EF, §3.1]) are a useful technical device both for the proof of Theorem 1.5 and for some of the arguments below. Two $(\kappa, \epsilon, \rho)$ graded q.i. embeddings $q_{1}$ and $q_{2}$ centered at the same point $z_{0}$ are equivalent if for all $x \in X_{1}, d\left(q_{1}(x), q_{2}(x)\right) \leq \max \left(\rho, \epsilon d\left(x, z_{0}\right)\right)$.

Neighborhoods, conical neighborhoods. We denote the $r$-tubular neighborhood of a set $U$ by $\operatorname{Nbhd}(U, r)$. The metric interior $\operatorname{Int}(U, r)$ is defined to be $U \backslash \operatorname{Nbhd}(\partial U, r)$.

Let $U$ be a subset of a metric space $Y$, and $y_{0} \in Y$ a point. We let $U\left[c ; y_{0}\right]$ be a neighborhood of $U$ whose size is proportional to the distance from $y_{0}$, namely:

$$
U\left[c ; y_{0}\right]=\left\{y \in Y \quad: \quad \exists u \in U \text { with } d(y, u)<c d\left(y, y_{0}\right)\right\} .
$$

As in [EF], if $U \subset \mathbb{R}^{n}$, we write $U[c]$ as shorthand for $U[c ; 0]$, and if $U \subset X$, we write $U[c]$ as shorthand for $U[c ; e]$.

Hausdorff distance and $\epsilon$-equivalence. If $U, V$ are subsets of a metric space, we denote the Hausdorff distance between their closures by $h d(U, V)$. If $h d(U, V)<\infty$, we say that $U$ is equivalent to $V$ and write $U \sim V$.

Let $x_{0}$ be a point and let $A_{R}\left(x_{0}\right)$ denote the annulus $\left\{x: R<d\left(x, x_{0}\right)<2 R\right\}$. We say that two subsets $U$ and $V$ are $\epsilon$-equivalent and write $U \sim_{\epsilon} V$ if for all sufficiently large $R$,

$$
h d\left(U \cap A_{R}\left(x_{0}\right), V \cap A_{R}\left(x_{0}\right)\right) \leq \epsilon R
$$

(clearly this is meaningful only if $\epsilon$ is small). 
Because of the triangle inequality, the notion of $\epsilon$-equivalence is (almost) independent of the point $x_{0}$. Indeed, if $U \sim_{\epsilon} V$ with origin $x_{0}$, and $z_{0}$ is any other point, then $U \sim_{\delta} V$ with origin $z_{0}$ for any $\delta>\epsilon$.

We will occasionally need a more quantitative version. We write $U \sim_{\epsilon, x_{0}, \rho} V$ if for all $R>\rho$, (4) holds. It is easy to check that if $U \sim_{\epsilon, x, \rho} V$, then outside the ball $B(x, \rho)$

$$
U \subset V[2 \epsilon ; x] \text { and } V \subset U[2 \epsilon ; x] .
$$

Conversely, if (5) holds outside the ball $B(x, \rho)$, then $U \sim_{4 \epsilon, x, \rho} V$.

Multiplicative constants depending only $\kappa$ and $X$. As in [EF], the argument produces a proliferation of constants depending only on $\kappa$ and $X$. (Essentially there is an extra factor of $\kappa$ each time (1) or (3) is used.) Thus we use the $\gg, \ll, \prec, \succ$ and $O(\cdot)$ notations in the same way as defined in [EF, §3.1].

1.3. Structure of the proof. The proof of Theorem 0.1 is a succession of steps; in each step we gain more control over the map $\phi$. Each step is proved in its own section; only the results stated in the introductory subsection of each section are used in subsequent sections.

We now begin the proof of Theorem 0.1. In view of Theorem 1.1, we may assume that $\phi$ is a $(\kappa, C)$ quasi-isometry $\Gamma \rightarrow \Gamma$ in the induced metric.

Step 1 (Extending $\phi$ to the "neutered space"). For any compact subset $\mathcal{C}$ of $\Gamma \backslash X$, $\phi$ can be extended to a $\left(\kappa, C^{\prime}\right)$ quasi-isometry of $\bar{p}^{-1}(\mathcal{C})$ to $\bar{p}^{-1}(\mathcal{C})$. Here $C^{\prime}$ depends on $\kappa, C$ and $\mathcal{C}$.

Proof. This construction is now standard; see e.g. [S1], [FS], [S2]. Since for every point $y$ of $p^{-1}(\mathcal{C})$ is within a uniformly bounded distance of a point in $\Gamma$, one may just define $\phi(y)$ to be $\phi(\gamma)$ where $\gamma$ is the point in $\Gamma$ closest to $y$ (one chooses randomly if there are several possibilities for $\gamma$ ). The resulting map is easily verified to be a quasi-isometry.

For notational convenience, we define $\phi$ on $\pi^{-1}\left(\bar{p}^{-1}(\mathcal{C})\right) \subset G$ by making $\phi$ constant along the $K$ fibers.

Choice of $\mathcal{C}$ and $\epsilon$. We choose $\epsilon>0$ so that Theorem 1.5 holds, and also so that $\epsilon \ll 1$, i.e. for any multiplicative constant $\lambda=\lambda(\kappa)$ which arises in $\S \S 2-7$ $\lambda \epsilon<1 / 100$. Note that $\epsilon$ depends only on $\kappa$.

We now choose $\mathcal{C}$ so that $\bar{\mu}(\mathcal{C})>1-\epsilon / 4$. The set $\mathcal{C}$ and the constant $\epsilon$ are fixed throughout the argument.

\section{QUASI-Flats WITH HOLES AND THE MOORE ERGODICITY THEOREM}

Definition 2.1 ( $\Gamma$-invariant family of flats). A family $\mathcal{F}$ of flats is $\Gamma$-invariant if for every $\gamma \in \Gamma$ and every flat $F \in \mathcal{F}, \gamma F \in \mathcal{F}$. A $\Gamma$-invariant family $\mathcal{F}$ of flats is said to have full measure if there exists a set $E \subset \Gamma \backslash G$ with $\mu(E)=1$ such that for any $y \in p^{-1}(E), \pi(y A) \in \mathcal{F}$.

The approach in this paper is based on the following lemma, which follows trivially from the ergodic theorem, Theorem 1.4:

Lemma 2.2. There exist constants $R>0, \rho>0$, a family $\mathcal{F}$ of flats in $X$ and for each $F \in \mathcal{F}$ subsets $\Omega_{F} \subset \Omega_{F}^{\prime} \subset F$ with the following properties:

(a) $\mathcal{F}$ is a $\Gamma$-invariant family of full measure. 
(b) For every $F \in \mathcal{F}, U_{F}=F \cap \bar{p}^{-1}(\mathcal{C})$ is not empty. In fact, for any $x \in F$,

$$
\liminf _{r \rightarrow \infty} \frac{\left|U_{F} \cap B(x, r)\right|}{|B(x, r)|} \geq 1-\frac{\epsilon}{4} .
$$

The quasi-isometry $\phi$ is defined on $U_{F}$.

(c) For every $F \in \mathcal{F}, \Omega_{F}^{\prime} \subset\left(U_{F}\right)_{(\epsilon, R)}$ is not empty. In fact, for any $x \in F$,

$$
\liminf _{r \rightarrow \infty} \frac{\left|\Omega_{F}^{\prime} \cap B(x, r)\right|}{|B(x, r)|} \geq 1-\frac{\epsilon}{2}
$$

where the notation $\left(U_{F}\right)_{(\epsilon, R)}$ is as in Theorem 1.5.

(d) For every $F \in \mathcal{F}, \Omega_{F} \subset\left(\Omega_{F}^{\prime}\right)_{(\epsilon, \rho)}$ is not empty. In fact, for any $x \in F$,

$$
\liminf _{r \rightarrow \infty} \frac{\left|\Omega_{F} \cap B(x, r)\right|}{|B(x, r)|} \geq 1-\frac{\epsilon}{2} .
$$

Remark. From (c) and Theorem 1.5, it follows that for all $F \in \mathcal{F}, \phi\left(\Omega_{F}^{\prime}\right)$ is close to a finite union of flats. Thus, in view of (6) we have information about the image under $\phi$ of "most of the volume in almost all flats".

We need (d) for a technical reason which will become apparent shortly.

Proof of Lemma 2.2. Let $\chi$ denote the characteristic function of the set $\pi^{-1}(\mathcal{C}) \subset$ $\Gamma \backslash G$. Then, in our normalizations, $\int_{\Gamma \backslash G} \chi d \mu \geq 1-\epsilon / 4$. Then by Theorem 1.4, there exists a set $E^{\prime} \subset \Gamma \backslash G$ such that $\mu\left(E^{\prime}\right)=1$, and for all $x \in E^{\prime}$,

$$
\lim _{r \rightarrow \infty} \frac{1}{\left|B_{r}\right|} \int_{B_{r}} \chi(x \exp v) d \nu(v) \geq 1-\epsilon / 4
$$

where $B_{r}$ denotes the ball $B(0, r) \subset \mathfrak{a}$.

Suppose $y \in p^{-1}\left(E^{\prime}\right)$, and let $F=\pi(y A)$. Note that for $v \in \mathfrak{a}$,

$$
\pi(y \exp v) \in U_{F}=F \cap \bar{p}^{-1}(\mathcal{C}) \quad \text { precisely when } \quad \chi(y \exp v)=1 .
$$

Thus if we let $\mathcal{F}_{1}=\left\{\pi(y A): y \in p^{-1}\left(E^{\prime}\right)\right\},(\mathrm{a})$-(b) of Lemma 2.2 hold for $\mathcal{F}_{1}$ instead of $\mathcal{F}$.

To get (c) we may argue as follows: For any $j>0$, let

$$
E_{j}^{\prime}=\left\{x \in E^{\prime}: \text { for all } r>j, \frac{1}{\left|B_{r}\right|} \int_{B_{r}} \chi(x \exp v) d \nu(v) \geq 1-\epsilon / 2\right\} .
$$

Then $E_{j}^{\prime}$ is an increasing family of sets and $\bigcup_{j=1}^{\infty} E_{j}^{\prime}=E^{\prime}$. Thus, there exists an integer $R$ such that $\mu\left(E_{R}^{\prime}\right) \geq 1-\epsilon / 4$. Let $E_{R}=E_{R}^{\prime} \cap \pi^{-1}(\mathcal{C})$; then $\mu\left(E_{R}\right) \geq 1-\epsilon / 2$.

Note that if $y \in p^{-1}\left(E^{\prime}\right)$ and $F=\pi(y A)$, then in view of equations (7) and (8), $\pi\left(y A \cap p^{-1}\left(E_{R}\right)\right) \subset\left(U_{F}\right)_{(\epsilon, R)}$. Thus we let $\Omega_{F}^{\prime}=\pi\left(y A \cap p^{-1}\left(E_{R}\right)\right)$.

Let $\chi_{R}$ denote the characteristic function of $E_{R}$. Then by the ergodic theorem, Theorem 1.4, there exists a set $E^{\prime \prime} \subset E^{\prime}$ with $\mu\left(E^{\prime \prime}\right)=1$ such that for $x \in E^{\prime \prime}$,

$$
\lim _{r \rightarrow \infty} \frac{1}{\left|B_{r}\right|} \int_{B_{r}} \chi_{R}(x \exp v) d \nu(v)=\mu\left(E_{R}\right) \geq 1-\epsilon / 2 .
$$

Thus we can let $\mathcal{F}_{2}=\left\{\pi(y A): y \in p^{-1}\left(E^{\prime \prime}\right)\right\}$. Since $\mu\left(E^{\prime \prime}\right)=1$, part (a) holds for $\mathcal{F}_{2}$. Since $\mathcal{F}_{2} \subset \mathcal{F}_{1}$, part (b) also holds. Finally part (c) holds for $\mathcal{F}_{2}$ in view of (9). 
To get $(\mathrm{d})$ we repeat the argument for $(\mathrm{c})$. Let

$$
E_{j}^{\prime \prime}=\left\{x \in E^{\prime \prime} \quad: \quad \text { for all } r>j, \frac{1}{\left|B_{r}\right|} \int_{B_{r}} \chi_{R}(x \exp v) d \nu(v) \geq 1-\epsilon\right\} .
$$

Then $E_{j}^{\prime \prime}$ is an increasing family, with $\bigcup_{j=1}^{\infty} E_{j}^{\prime \prime}=E^{\prime \prime}$. Thus there exists an integer $\rho$ such that $\mu\left(E_{\rho}^{\prime \prime}\right) \geq 1-\epsilon / 4$. Let $\Omega=E_{\rho}^{\prime \prime} \cap \pi^{-1}(\mathcal{C})$; then $\mu(\Omega) \geq 1-\epsilon / 2$. For $F=\pi(y A) \in \mathcal{F}_{2}$, we set $\Omega_{F}=\pi(y A \cap \Omega)$. Then in view of $(10), \Omega_{F} \subset\left(\Omega_{F}^{\prime}\right)_{(\epsilon, \rho)}$. Let $\chi_{\Omega}$ denote the characteristic function of $\Omega$. Thus $\pi(y \exp v) \in \Omega_{F}$ precisely when $p(y \exp v) \in \Omega$.

By Theorem 1.4, there exists a subset $E^{\prime \prime \prime} \subset E^{\prime \prime}$ with $\mu\left(E^{\prime \prime \prime}\right)=1$ such that for any $x \in E^{\prime \prime \prime}$,

$$
\lim _{r \rightarrow \infty} \frac{1}{\left|B_{r}\right|} \int_{B_{r}} \chi_{\Omega}(x \exp v) d \nu(v)=\mu(\Omega) \geq 1-\epsilon / 2 .
$$

Thus we can let $\mathcal{F}=\left\{\pi(y A): y \in p^{-1}\left(E^{\prime \prime \prime}\right)\right\}$. Part (a) holds since $\mu\left(E^{\prime \prime \prime}\right)=1$, parts (b)-(c) hold since $\mathcal{F} \subset \mathcal{F}_{2}$, and part (d) holds because of (11).

Combining Lemma 2.2 with Theorem 1.5 we get the following:

Step 2 ( $\phi$ maps almost all flats to finite unions of flats). There exist constants $M$ $=M(\kappa), \rho=\rho(\kappa, \epsilon, C)$ and $N=N(\kappa, \epsilon, C, \rho)$, a family of flats $\mathcal{F}$, and for each $F \in \mathcal{F}$ nonempty subsets $\Omega_{F} \subset \Omega_{F}^{\prime} \subset F$, such that the following hold:

(a) $\mathcal{F}$ is a $\Gamma$-invariant family of full measure.

(b) For every $F \in \mathcal{F}$, there exist at most $M$ flats $F_{1}^{\prime}, \ldots, F_{M}^{\prime}$ such that $\phi\left(\Omega_{F}^{\prime}\right)$ lies within a distance $N$ of $\bigcup_{j=1}^{M} F_{j}^{\prime}$.

(c) For every $F \in \mathcal{F}$, every $x \in \Omega_{F}$, and every $r>\rho$,

$$
\frac{\left|B(x, r) \cap \Omega_{F}^{\prime}\right|}{|B(x, r)|} \geq 1-\epsilon .
$$

Hence there exists a map $\tilde{\phi}: F \rightarrow \operatorname{Nbhd}\left(\bigcup_{j=1}^{M} F_{j}^{\prime}, N\right)$, which agrees with $\phi$ on $\Omega_{F}^{\prime}$, and is a $(\kappa, 2 \kappa \epsilon, \rho)$ graded quasi-isometric embedding centered at $x$ for any $x \in \Omega_{F}$.

(d) For every $F \in \mathcal{F}$, and any $x \in F$,

$$
\liminf _{r \rightarrow \infty} \frac{\left|\Omega_{F} \cap B(x, r)\right|}{|B(x, r)|} \geq 1-\epsilon .
$$

(e) There exists a subset $\Omega \subset \Gamma \backslash G$ with $\mu(\Omega) \geq 1-\epsilon / 2$ such that for any $x \in p^{-1}(\Omega), F=\pi(x A) \in \mathcal{F}$ and $\pi(x) \in \Omega_{F}$.

The map $\tilde{\phi}$ of part (c) is just the map obtained by $\tilde{\phi}(u)=\phi(v)$ where $v$ is a point of $\Omega_{F}^{\prime}$ nearest $u$ (cf. Step 1). In view of $(12), \tilde{\phi}$ is indeed a $(\kappa, 2 \kappa \epsilon, \rho)$ graded q.i. embedding.

The set $\Omega$ of part (e) is the same as in the proof of Lemma 2.2. Part (e) will be used in $\S 7$ to "return from the boundary".

\section{MAPPing Flats to Flats}

Step 3 ( $\phi$ maps most flats to single flats). There exists a full-measure $\Gamma$-invariant family of flats $\mathcal{F}^{*} \subset \mathcal{F}$ such that for every $F \in \mathcal{F}^{*}$ the following hold: 
(i) Parts (b) and (c) of Step 2 hold with $M=1$; i.e. there exists a flat $F^{\prime}$ such that $\phi\left(\Omega_{F}^{\prime}\right) \subset \operatorname{Nbhd}\left(F^{\prime}, N\right)$ (the sets $\Omega_{F}, \Omega_{F}^{\prime}$ and the constant $N$ are the same as in Step 2).

(ii) There exists a constant $\lambda=\lambda(\kappa)$ such that for every Weyl chamber $\mathfrak{C} \subset F$ there exists a chamber $\mathfrak{C}^{\prime} \subset F^{\prime}$ such that $\phi\left(\mathfrak{C} \cap \Omega_{F}^{\prime}\right) \sim_{\lambda \epsilon} \mathfrak{C}^{\prime}$.

Remark. The proof of this step is somewhat involved.

Transverse flats. For each $\alpha \in \Sigma$, we pick $k_{\alpha} \in K$ such that:

$$
k_{\alpha} A k_{\alpha}^{-1} \cap A=A_{\alpha}=\{a \in A: \alpha(a)=0\} .
$$

We fix the $k_{\alpha}$ for the remainder of the paper. Now if $F=\pi(z A)$ is a flat, and $x=\pi(z a) \in F$, then we define

$$
\operatorname{trans}_{\alpha}(x ; z)=\pi\left(z a k_{\alpha} A\right) .
$$

Then $F_{\alpha}=\operatorname{trans}_{\alpha}(x ; z)$ is a flat, which intersects $F$ in the hyperplane $L_{\alpha}=$ $\pi\left(z a A_{\alpha}\right)$ passing through $x$. Let

$$
\operatorname{Trans}_{F}(x)=\left\{\operatorname{trans}_{\alpha}(x ; z): \alpha \in \Sigma, \text { and } F=\pi(z A)\right\} .
$$

We call any flat $F_{0} \in \operatorname{Trans}_{F}(x)$ a transverse flat to $F$ at $x$. The name is motivated by the following lemma:

Lemma 3.1 (Transverse flats). Suppose $F$ is a flat, $x \in F$, and $F_{0} \in \operatorname{Trans}_{F}(x)$. Then

(a) There exists a hyperplane $L \subset F$ with $x \in L$ and $F \cap F_{0}=L$.

(b) There exist constants $\lambda_{0}, \lambda_{1}$ depending only on $X$ (and the choice of the $k_{\alpha}$ ) such that for $r>\lambda_{0}$,

$$
h d\left(F \cap \operatorname{Nbhd}\left(F_{0}, r\right), L\right) \leq \lambda_{1} r .
$$

This lemma expresses in a quantitative way the fact that the flats $F$ and $F_{0}$ come close only near $L$. Part (a) follows from the definition of $k_{\alpha}$. Part (b) is essentially the fact that geodesics in $F_{1}$ and $F_{2}$ emanating from a point of $L$ and orthogonal to $L$ diverge; cf. the discussion at the beginning of $\S 5$.

The sets $\Omega_{z}^{*}$ and $\Omega_{F}^{*}$. Suppose $F=\pi(z A) \in \mathcal{F}$. Let

$$
\Omega_{z}^{*}=\left\{x \in \Omega_{F} \quad: \operatorname{trans}_{\alpha}(x ; z) \in \mathcal{F} \text { and } x \in \Omega_{\operatorname{trans}_{\alpha}(x ; z)} \text { for all } \alpha \in \Sigma\right\} .
$$

Hence if $x \in \Omega_{z}^{*}$, then for every hyperplane $L$ passing through $x$ there exists a flat $F_{L} \in \operatorname{Trans}_{F}(x)$ such that $x \in \Omega_{F_{L}}$.

As we defined it, the set $\Omega_{z}^{*}$ depends on $z$, and not only on $F=\pi(z A)$. Even though it is not essential for the argument, to simplify some notation we set

$$
\Omega_{F}^{*}=\underset{\{z \text { with } \pi(z A)=F\}}{\bigcup_{z}^{*} .} \Omega
$$

Thus $\Omega_{F}^{*} \subset \Omega_{F} \subset \Omega_{F}^{\prime} \subset F$.

Lemma 3.2 (the family $\mathcal{F}^{*}$ ). There exists a full-measure $\Gamma$-invariant family $\mathcal{F}^{*} \subset$ $\mathcal{F}$ such that for any $F \in \mathcal{F}^{*}$ and any $x \in F$,

$$
\liminf _{r \rightarrow \infty} \frac{\left|\Omega_{F}^{*} \cap B(x, r)\right|}{|B(x, r)|} \geq 1-|\Sigma| \epsilon .
$$


Proof. Let

$$
\Omega^{*}=\bigcap_{\alpha \in \Sigma} \Omega k_{\alpha}^{-1}
$$

where $\Omega$ is as in the proof of Lemma 2.2. Then $\mu\left(\Omega^{*}\right) \geq 1-|\Sigma| \epsilon$. Suppose $z \in G$. Note that if $p(z a) \in \Omega^{*}$ for some $a \in A$, then $p\left(z a k_{\alpha}\right) \in \Omega$ for all $\alpha \in \Sigma$, hence $x=\pi(z a)=\pi\left(z a k_{\alpha}\right) \in \Omega_{\pi\left(z a k_{\alpha} A\right)}=\Omega_{\text {trans }_{\alpha}(x ; z)}$ for all $\alpha \in \Sigma$. Hence:

$$
x=\pi(z a) \in \Omega_{z}^{*} \quad \text { if } p(z a) \in \Omega^{*} .
$$

Let $\chi_{\Omega^{*}}$ be the characteristic function of $\Omega^{*}$. By the ergodic theorem, Theorem 1.4, there exists a set $E^{*} \subset E^{\prime \prime \prime} \subset \Gamma \backslash G$ with $\mu\left(E^{*}\right)=1$ (where $E^{\prime \prime \prime}$ is as in the proof of Lemma 2.2) such that for all $x \in E^{*}$,

$$
\lim _{r \rightarrow \infty} \frac{1}{\left|B_{r}\right|} \int_{B_{r}} \chi_{\Omega^{*}}(x \exp v) d \nu(v)=\mu\left(\Omega^{*}\right) \geq 1-|\Sigma| \epsilon .
$$

Let $\mathcal{F}^{*}=\left\{\pi(z A) \quad: \quad z \in p^{-1}\left(E^{*}\right)\right\}$. Then for $F \in \mathcal{F}^{*}$ Lemma 3.2 holds in view of (15) and (16).

Step 3 will follow from the following:

Proposition 3.3. Let $\mathcal{F}^{*}$ be the family defined in Lemma 3.2. Then for every flat $F \in \mathcal{F}^{*}$, (i) and (ii) of Step 3 hold.

The proof of Proposition 3.3, which involves no ergodic theory, occupies the rest of this section. Along the way, we will prove the following result, which will be used in $\S 5$ :

Proposition 3.4. There exist constants $\lambda=\lambda(\kappa)$ and $\rho^{\prime}=\rho^{\prime}(\kappa, C, \epsilon, X)$ such that the following holds: Suppose $F \in \mathcal{F}^{*}$, and $x \in \Omega_{F}^{*}$. Then:

(a) For every hyperplane $L \subset F$ passing through $x$ there exists a hyperplane $L^{\prime}$ such that $\tilde{\phi}(L) \sim_{\lambda \epsilon, \tilde{\phi}(x), \rho^{\prime}} L^{\prime}$.

(b) For every chamber $\mathfrak{C} \subset F$ based at $x$ there exists a chamber $\mathfrak{C}^{\prime}$ such that $\tilde{\phi}(\mathfrak{C}) \sim_{\lambda \epsilon, \tilde{\phi}(x), \rho^{\prime}} \mathfrak{C}^{\prime}$.

3.1. Intersections of quasi-flats. Our aim in this subsection is to prove the following:

Lemma 3.5 (Hyperplanes map to finite unions of hyperplanes). Suppose $F \in \mathcal{F}^{*}$, $x_{0} \in F$ and $R$ is sufficiently large (depending on $F$ ). Let $A_{R}=A\left(R, 2 R ; \phi\left(x_{0}\right)\right) \subset$ $X$. Then there exists $M^{\prime}>0$ (depending only on $\kappa, X$ ) such that for any hyperplane $L$ in $F$, there exist at most $M^{\prime}$ hyperplanes $L_{1}^{\prime}, \ldots, L_{M^{\prime}}^{\prime}$ such that

$$
\tilde{\phi}(L) \cap A_{R} \subset \operatorname{Nbhd}\left(\bigcup_{j=1}^{M^{\prime}} L_{j}^{\prime}, O(\epsilon R)\right) \cap A_{R} .
$$

The proof uses the following lemma:

Lemma 3.6 (Quasi-flats are unions of polyhedra). Suppose $F \in \mathcal{F}, x \in \Omega_{F}$ and $R>\lambda^{\prime} \rho$ (where $\rho$ is as in Step 2 and $\lambda^{\prime}=\lambda^{\prime}(\kappa)$ ). Let $B_{R}$ denote $B(\phi(x), R)$. Then there exist $m=m(\kappa)$ and $\lambda=\lambda(\kappa)$ and convex polyhedra $\mathcal{P}_{1}, \ldots, \mathcal{P}_{m}$ such that

$$
h d\left(\tilde{\phi}(F) \cap B_{R}, \bigcup_{j=1}^{m} \mathcal{P}_{j} \cap B_{R}\right)<\lambda \epsilon R .
$$


Remark. If instead of the assumption $R \gg \rho$ we made the assumption " $R$ sufficiently large depending on $F$ ", Lemma 3.6 would follow from [EF, Lemma 8.3] (restated as Lemma 3.11 below). For the case of nongraded quasi-isometries, intersecting with a ball is not necessary. Thus Lemma 3.6 can be viewed as a strengthening of [EF, Lemma 8.3] in the sense that the Hausdorff distance from a quasi-flat to some explicitly given set is bounded only in terms of the quasi-isometry constants.

Proof of Lemma 3.6. By Step 2 we have a $(\kappa, 2 \kappa \epsilon, \rho)$ graded quasi-isometry centered at $x, \tilde{\phi}: F \rightarrow \operatorname{Nbhd}\left(\bigcup_{j=1}^{M} F_{j}^{\prime}, N\right)$.

Fix $\eta^{\prime \prime} \gg \eta \gg \eta^{\prime} \gg 1$. In the proof of this lemma, all implicit constants depend only on $\kappa$ and $X$, and the dependence on $\eta^{\prime \prime}, \eta$ and $\eta^{\prime}$ is shown explicitly.

Let $\lambda_{0}$ be as in Lemma B.1, part (i). For each pair $(i, j)$ there is an interval $I_{i j}=\left[d\left(F_{i}^{\prime}, F_{j}^{\prime}\right), \lambda_{0}\left(1+d\left(F_{i}^{\prime}, F_{j}^{\prime}\right)\right)\right]$ for which Lemma B.1 cannot be applied. Since the number of flats $M$ is $O(1)$ (i.e. depends only $\kappa$ and $X$ ), we may choose $r_{0}=$ $O\left(\eta^{\prime \prime} \epsilon R\right)$ such that $r_{0} \gg \epsilon R$ and $\left[r_{0}, \eta^{\prime \prime} r_{0}\right]$ does not intersect any $I_{i j}$. For example, $r_{0}=r_{0}\left(\eta^{\prime \prime} \in R\right)$ can be constructed as follows: Denote by $I$ the union of the half-open intervals $\left(\frac{1}{\eta^{\prime \prime}} d\left(F_{i}^{\prime}, F_{j}^{\prime}\right), \lambda_{0}\left(1+d\left(F_{i}^{\prime}, F_{j}^{\prime}\right)\right]\right.$. Then let $r_{0}(t), t \in(0, \infty)$ be a piecewise linear function (with slope 1) continuous from the right, and with jumps such that the range of $r_{0}(t)$ is $(0, \infty) \backslash I$. Note that $t \leq r_{0}(t) \leq \lambda_{1}\left(\eta^{\prime \prime}\right)^{M(M-1) / 2} t$ where $\lambda_{1}$ depends only on $\lambda_{0}$ and $M$.

For $r_{0}<r<\eta^{\prime \prime} r_{0}$, let $\mathcal{P}_{i j}(r)$ be as in Lemma B.1, part (i), with $F_{1}=F_{i}^{\prime}$, $F_{2}=F_{j}^{\prime}$ if $r_{0}>\lambda_{0}\left(1+d\left(F_{i}^{\prime}, F_{j}^{\prime}\right)\right)$, and let $\mathcal{P}_{i j}(r)=\emptyset$ if $d\left(F_{i}^{\prime}, F_{j}^{\prime}\right)>\eta^{\prime \prime} r_{0}$. Note that $\mathcal{P}_{i i}(r)=F_{i}^{\prime}$.

For $r \in\left[r_{0}, \eta r_{0}\right], s \in\left[r_{0}, \eta r_{0}\right], s<r$ and $\sigma \subset\{1, \ldots, M\}$ let

$$
\mathcal{P}_{i, \sigma}(s, r)=\bigcap_{j \in \sigma} \mathcal{P}_{i j}(s) \bigcap_{j \notin \sigma} \mathcal{P}_{i j}(r)^{c} .
$$

Note that

$$
F_{i}^{\prime}=\bigcup_{\sigma \subset\{1, \ldots, M\}} \mathcal{P}_{i, \sigma}(s, r) \backslash \bigcup_{j=1}^{M}\left(\mathcal{P}_{i j}(r) \backslash \mathcal{P}_{i j}(s)\right) .
$$

From its definition $\mathcal{P}_{i j}(r) \backslash \mathcal{P}_{i j}(s)$ is contained in a union of neighborhoods of hyperplanes, each of width $O\left(\eta r_{0}\right)$. Hence, for each $i$,

$$
h d\left(F_{i}^{\prime}, \underset{\sigma \subset\{1, \ldots, M\}}{\bigcup} \mathcal{P}_{i, \sigma}(s, r)\right)=O\left(\eta r_{0}\right) .
$$

For any $a<b$, let

$$
F_{i, \sigma}^{\prime}(a, b)=\left\{x \in F_{i}^{\prime} \quad: d\left(x, F_{j}^{\prime}\right) \leq a, \quad \forall j \in \sigma \quad \text { and } \quad d\left(x, F_{j}\right)>b, \quad \forall j \notin \sigma\right\} .
$$

Note that $F_{i, \sigma}^{\prime}=\emptyset$ if $i \notin \sigma$. Let $\lambda_{1}, \lambda_{2}$ be as in Lemma B.1, part (i). Then, by Lemma B.1,

$$
\bigcap_{j \in \sigma} \mathcal{P}_{i j}\left(\lambda_{1} a\right) \bigcap_{j \notin \sigma} \mathcal{P}_{i j}\left(\lambda_{2} b\right)^{c} \subset F_{i, \sigma}^{\prime}(a, b) \subset \bigcap_{j \in \sigma} \mathcal{P}_{i j}\left(\lambda_{2} a\right) \bigcap_{j \notin \sigma} \mathcal{P}_{i j}\left(\lambda_{1} b\right)^{c} .
$$

Hence,

$$
\mathcal{P}_{i, \sigma}\left(\lambda_{1} a, \lambda_{2} b\right) \subset F_{i, \sigma}^{\prime}(a, b) \subset \mathcal{P}_{i, \sigma}\left(\lambda_{2} a, \lambda_{1} b\right) .
$$


The hyperplanes bounding $\mathcal{P}_{i, \sigma}(s, r)$ subdivide $F_{i}^{\prime}$ into convex polyhedra; for each pair $(s, r)$ there is a certain combinatorial pattern (i.e. which hyperplanes bound which convex polyhedra). The possible number of patterns is bounded depending only on $X$, and it is easy to see that the set of $(s, r)$ which gives rise to a fixed pattern is a convex subset of $\mathbb{R}^{2}$. Thus we have a decomposition of the square $\left[r_{0}, \eta r_{0}\right]^{2}$ into a finite number of disjoint convex sets. Hence, if $\eta \gg \eta^{\prime}$ is sufficiently large, one of the sets must contain a box of side length $\eta r_{0} / \eta^{\prime}$, i.e. there exists $\left(s_{1}, r_{1}\right) \in\left[r_{0}, \eta r_{0}\right]^{2}$ such that the combinatorial pattern is the same for all $(s, r) \in\left[s_{1} / \eta^{\prime}, s_{1}\right] \times\left[r_{1} / \eta^{\prime}, r_{1}\right]$. Without loss of generality, we may assume that $r_{1}>s_{1}$. Hence,

$$
h d\left(\mathcal{P}_{i, \sigma}(s, r), \mathcal{P}_{i, \sigma}\left(s_{1}, r_{1}\right)\right)=O\left(r_{1}\right) \quad \text { for all }(s, r) \in\left[s_{1} / \eta^{\prime}, s_{1}\right] \times\left[r_{1} / \eta^{\prime}, r_{1}\right] .
$$

Suppose $a \in\left[2 s_{1} /\left(\lambda_{1} \eta^{\prime}\right), s_{1} /\left(2 \lambda_{2}\right)\right]$ and $b \in\left[2 r_{1} /\left(\lambda_{1} \eta^{\prime}\right), r_{1} /\left(2 \lambda_{2}\right)\right]$. Then by $(20)$ and (19)

$$
h d\left(F_{i, \sigma}^{\prime}(a, b), \mathcal{P}_{i, \sigma}\left(s_{1}, r_{1}\right)\right)=O\left(r_{1}\right)=O\left(\eta r_{0}\right) .
$$

By (18), for any $i, j \in \sigma, F_{i, \sigma}^{\prime}(a, b) \subset \operatorname{Nbhd}\left(F_{j, \sigma}^{\prime}(2 a, b-a), a\right)$. Hence, by (21),

$$
h d\left(\mathcal{P}_{i, \sigma}\left(s_{1}, r_{1}\right), \mathcal{P}_{j, \sigma}\left(s_{1}, r_{1}\right)\right)=O\left(r_{1}\right)=O\left(\eta r_{0}\right) \quad \text { for all } i, j \in \sigma .
$$

Write $\mathcal{P}_{\sigma}=\mathcal{P}_{i, \sigma}\left(s_{1}, r_{1}\right)$ where $i=\min _{j \in \sigma} j$. Hence, combining (22) and (17),

$$
h d\left(\bigcup_{j=1}^{M} F_{j}^{\prime}, \bigcup_{\sigma \subset\{1, \ldots, M\}} \mathcal{P}_{\sigma}\right)=O\left(\eta r_{0}\right) .
$$

Hence (after changing $\tilde{\phi}$ by $O\left(\eta r_{0}\right)$ ) we may view $\tilde{\phi}$ as a $\left(\kappa, C^{\prime}\right)$ quasi-isometry $\tilde{\phi}: B \rightarrow \bigcup \mathcal{P}_{\sigma}$, where $C^{\prime}=O\left(\eta r_{0}\right)$ and $B$ is a ball containing $\phi^{-1}\left(B_{R}\right)$. We would like to apply Lemma A.3; we now verify that for any $r \gg \eta r_{0}$ the estimate (46) is satisfied for (the convex pieces of) the $\mathcal{P}_{\sigma}$, with $C^{\prime}$ in place of $C$. First suppose $\sigma$ and $\tau$ are not disjoint; choose $j \in \sigma \cap \tau$. By (22), the Hausdorff distance from $\mathcal{P}_{\sigma}$ to $\mathcal{P}_{j, \sigma}\left(s_{1}, r_{1}\right)$ is $O\left(\eta r_{0}\right)$, and the Hausdorff distance from $\mathcal{P}_{\tau}$ to $\mathcal{P}_{j, \tau}\left(s_{1}, r_{1}\right)$ is also $O\left(\eta r_{0}\right)$. But $\mathcal{P}_{j, \sigma}\left(s_{1}, r_{1}\right)$ and $\mathcal{P}_{j, \tau}\left(s_{1}, r_{1}\right)$ are disjoint polyhedra lying in the flat $F_{j}^{\prime}$. Then, the required estimate clearly holds for any $r \gg C^{\prime}$. If $\sigma$ and $\tau$ are disjoint, then pick $j \in \tau \backslash \sigma$. Since

$$
\operatorname{Int}\left(\mathcal{P}_{i, \sigma}\left(s_{1}, r_{1}\right), r\right) \subset \mathcal{P}_{i, \sigma}\left(s_{1}, r_{1}+r\right) \subset F_{i, \sigma}^{\prime}\left(\lambda_{1}^{-1} s_{1}, \lambda_{2}^{-1}\left(r_{1}+r\right)\right)
$$

for any $x \in \operatorname{Int}\left(\mathcal{P}_{i, \sigma}, r\right)$, and since $j \notin \sigma$, we have $d\left(x, F_{j}^{\prime}\right) \geq \lambda_{2}^{-1}\left(r_{1}+r\right) \geq \lambda_{2}^{-1} r$. Hence, for any $y \in B\left(x, 4 \kappa \eta_{1} \eta_{2} C^{\prime}\right), d\left(y, F_{j}^{\prime}\right) \geq \lambda_{2}^{-1} r-4 \kappa \eta_{1} \eta_{2} C^{\prime} \succ r$ if $r \gg C^{\prime}$. But, by (19), for any $z \in \mathcal{P}_{\tau}, d\left(z, F_{j}\right)=O\left(\eta r_{0}\right)=O\left(C^{\prime}\right)$. Hence, $y \notin \mathcal{P}_{\tau}$. Thus (46) holds. Now the lemma follows from Lemma A.3.

Lemma 3.7 (Closeness sets of polyhedra). Suppose $F_{1}, F_{2} \in \mathcal{F}$ are flats, $x \in$ $\Omega_{F_{1}} \cap \Omega_{F_{2}}$ and $F_{2} \in \operatorname{Trans}_{F_{1}}(x)$. Choose any $R$ such that $R \gg \rho$, and write $B_{R}$ for $B(\tilde{\phi}(x), R)$. Let $\mathcal{P}_{1}, \mathcal{P}_{2} \subset X$ be polyhedra and suppose there exist subsets $U_{i} \in F_{i}$ such that $h d\left(\tilde{\phi}\left(U_{i}\right), \mathcal{P}_{i} \cap B_{R}\right)=O(\epsilon R)$. Then, for any $r$ with $\epsilon R \ll r \ll R$ :

(a) The injectivity radius of $\mathcal{P}_{1} \cap \operatorname{Nbhd}\left(\mathcal{P}_{2}, r\right) \cap B_{R}$ is $O(r)$.

(b) There exists a hyperplane $L \subset \mathcal{P}_{1}$ such that

$$
\mathcal{P}_{1} \cap \operatorname{Nbhd}\left(\mathcal{P}_{2}, r\right) \cap B_{R} \subset \operatorname{Nbhd}(L, O(r)) \cap B_{R} .
$$


Proof. Let $d_{0}=d\left(F_{1}^{\prime}, F_{2}^{\prime}\right)$ where the $F_{j}^{\prime}$ are flats containing $\mathcal{P}_{j}$. Let $d(r)=r$ when $0<r<d_{0}$, and $d(r)=\lambda_{0}\left(1+d_{0}\right)+r-d_{0}$ when $r \geq d_{0}$. Then $r \leq d(r) \leq \lambda_{0}(r+1)$ and the range of $d(r)$ does not contain the interval $\left[d_{0}, \lambda_{0}\left(1+d_{0}\right)\right)$ where Lemma B.1 cannot be applied. If $\epsilon R \ll r \ll R$, then $d(r)=O(r)$ and by Lemma B.1, part (iii), there exists a polyhedron $\mathcal{Q} \subset \mathcal{P}_{1}$ such that $h d\left(\mathcal{Q}, \mathcal{P}_{1} \cap \operatorname{Nbhd}\left(\mathcal{P}_{2}, d(r)\right)\right)=O(r)$.

By assumption, $\tilde{\phi}$ has a coarse inverse $\phi^{*}:\left(\mathcal{P}_{1} \cup \mathcal{P}_{2}\right) \cap B_{R} \rightarrow U_{1} \cup U_{2}$, which is a $\left(\kappa, C^{\prime}\right)$ quasi-isometric embedding with $C^{\prime}=O(\epsilon R)$. By Lemma A.4,

$$
\begin{array}{r}
\phi^{*}\left(\mathcal{P}_{1} \cap \operatorname{Nbhd}\left(\mathcal{P}_{2}, d(r)\right) \cap B_{R}\right) \subset \operatorname{Nbhd}\left(U_{1}, O(r)\right) \cap \operatorname{Nbhd}\left(U_{2}, O(r)\right) \\
\subset \operatorname{Nbhd}\left(F_{1}, O(r)\right) \cap \operatorname{Nbhd}\left(F_{2}, O(r)\right) .
\end{array}
$$

By Lemma 3.1, the injectivity radius of $\operatorname{Nbhd}\left(F_{1}, O(r)\right) \cap \operatorname{Nbhd}\left(F_{2}, O(r)\right)$ is $O(r)$. Hence, since $\phi^{*}$ is a quasi-isometry, the injectivity radius of $\mathcal{P}_{1} \cap \operatorname{Nbhd}\left(\mathcal{P}_{2}, d(r)\right) \cap B_{R}$ and hence of $\mathcal{Q}$ is $O(r)$. Now the assertion (b) follows from Lemma B.9 and the fact that $d(r) \geq r$.

Proof of Lemma 3.5. Choose $R_{0}$ sufficiently large so that for any $y \in F$ outside $B_{R_{0}}$, there exists a point $x \in \Omega_{F}^{*}$ with $d(x, y)=O\left(\epsilon d\left(y, x_{0}\right)\right)$, and suppose $R>$ $4 \kappa R_{0}$.

If $L$ does not intersect $B=B\left(x_{0}, 4 \kappa R\right)$, then $\tilde{\phi}(L)$ does not intersect $B\left(\phi\left(x_{0}\right), 2 R\right)$ and there is nothing to prove. If $L$ does intersect $B$, then we can replace $L$ by a parallel hyperplane $L_{1}$ such that $h d\left(L \cap B, L_{1} \cap B\right)=O(\epsilon R)$, and $L_{1}$ contains a point $x \in \Omega_{F}^{*}$. Choose $F_{0} \in \operatorname{Trans}_{F}(x)$ such that $F \cap F_{0}=L_{1}$.

By Lemma 3.6, there exist finite unions of polyhedra, $\mathcal{P}_{1}, \ldots, \mathcal{P}_{M}$ and $\mathcal{Q}_{1}, \ldots, \mathcal{Q}_{M}$ such that $h d\left(\tilde{\phi}(F) \cap A_{R}, \bigcup \mathcal{P}_{i} \cap A_{R}\right)=O(\epsilon R)$ and $h d\left(\tilde{\phi}\left(F_{0}\right) \cap A_{R}, \bigcup \mathcal{Q}_{j} \cap A_{R}\right)=$ $O(\epsilon R)$. By Lemma A.4,

$$
\begin{aligned}
\tilde{\phi}\left(L_{1}\right) \cap A_{R} \subset \operatorname{Nbhd} & \left(\bigcup_{i=1}^{M} \mathcal{P}_{i}, O(\epsilon R)\right) \cap \operatorname{Nbhd}\left(\bigcup_{j=1}^{M} \mathcal{Q}_{j}, O(\epsilon R)\right) \cap A_{R} \\
& \subset \bigcup_{i=1}^{M} \bigcup_{j=1}^{M} \operatorname{Nbhd}\left(\mathcal{P}_{i}, O(\epsilon R)\right) \cap \operatorname{Nbhd}\left(\mathcal{Q}_{j}, O(\epsilon R)\right) \cap A_{R} .
\end{aligned}
$$

But by Lemma 3.7 (b) with $U_{1}=\tilde{\phi}^{-1}\left(\mathcal{P}_{i} \cap B_{4 R}\right), U_{2}=\tilde{\phi}^{-1}\left(\mathcal{Q}_{j} \cap B_{4 R}\right)$, for every $i, j$ there exists a hyperplane $L_{i j}^{\prime} \subset \mathcal{P}_{i}$ such that

$$
\operatorname{Nbhd}\left(\mathcal{P}_{i}, O(\epsilon R)\right) \cap \operatorname{Nbhd}\left(\mathcal{Q}_{j}, O(\epsilon R)\right) \cap A_{R} \subset \operatorname{Nbhd}\left(L_{i j}^{\prime}, O(\epsilon R)\right) \cap A_{R} .
$$

This implies that the lemma holds for $L_{1}$. Hence it also holds for $L$.

The same argument as in the proof of Lemma 3.5 also shows the following variant of Lemma 3.5:

Lemma 3.8. Suppose $F$ is a flat such that $\tilde{\phi}(F)$ is close to a single flat $F^{\prime}$ (in the sense of Step 2(c)). Suppose $x \in \Omega_{F}^{*}$, and $L$ is a hyperplane in $F$ passing through $x$. Suppose $R \gg \rho$, where $\rho$ is as in Step 2. Let $A_{R}=A(R, 2 R ; \phi(x)) \subset X$. Then there exists $M^{\prime}>0$ (depending only on $\kappa, X$ ) such that there exist at most $M^{\prime}$ hyperplanes $L_{1}^{\prime}, \ldots, L_{M^{\prime}}^{\prime} \subset F^{\prime}$ with

$$
\tilde{\phi}(L) \cap A_{R} \subset \operatorname{Nbhd}\left(\bigcup_{j=1}^{M^{\prime}} L_{j}^{\prime}, O(\epsilon R)\right) \cap A_{R} .
$$




\subsection{A certain class of quasi-isometries $\mathbb{R}^{n} \rightarrow \mathbb{R}^{n}$.}

Lemma 3.9 (Complexes). Let $S_{1}, \ldots, S_{r}$ be $r$ fixed hyperplanes passing through the origin in $\mathbb{R}^{n}$; their intersections form a cellular complex $Y$, whose maximal cells have dimension $n-1$. Suppose $f$ is a 1-1 cellular map from $Y$ to $Y$ (i.e. $f$ maps cells to cells, and preserves adjacency relations). Then either $f$ sends each hyperplane to a hyperplane, or there exist distinct hyperplanes $L$ and $M$ such that $f(L)$ separates $\mathbb{R}^{n}$ into two connected components $H_{1}$ and $H_{2}$, and $f(M)$ lies entirely in the closure of $H_{1}$.

Proof. We may assume that $f$ is a continuous map $Y \rightarrow Y$ which respects the simplicial complex structure. By the Jordan curve theorem, $f(L)$ separates $\mathbb{R}^{n}$ into two connected components $H_{1}$ and $H_{2}$. Suppose $f(L)$ is not a hyperplane. Then there exist two adjacent cells $C_{1}$ and $C_{2}$ in $L$ such that $f\left(C_{1}\right)$ and $f\left(C_{2}\right)$ do not lie in the same hyperplane. Since $C_{1}$ and $C_{2}$ are adjacent, there exists a subspace $C$ of codimension 2 containing $C_{1} \cap C_{2}$. Pick $x_{0} \in C_{1} \cap C_{2}$ away from the origin, and let $H_{0}$ be a 2-dimensional plane passing through $f\left(x_{0}\right)$ and orthogonal to $f(C)$. Denote by $D_{0}=D_{0}(\epsilon R)$ a disk in $H_{0}$ centered at $x_{0}$ and of radius $\epsilon R$ where $R$ is the distance from $x_{0}$ to the origin and $\epsilon>0$ is small.

Then for any hyperplane $M \supset C, f(M) \cap D_{0}$ is a "broken line", i.e. a onedimensional piecewise straight curve, with the (possible) turn at $f\left(x_{0}\right)$. For $M=L$, there is indeed a "turn" at $f\left(x_{0}\right)$, i.e. $f(L) \cap D_{0}$ consists of two half-lines intersecting at $f\left(x_{0}\right)$. Note that $f(L) \cap D_{0}$ divides $D_{0}$ into two open connected components $D_{1} \subset H_{1}$ and $D_{2} \subset H_{2}$, and the number of half-lines in $D_{1}$ and $D_{2}$ differs by at least 2. For definiteness suppose the number of half-lines in $D_{1}$ is larger than in $D_{2}$. Then for at least one hyperplane $M$ containing $C, f(M) \cap D_{0}$ is contained entirely in $\bar{D}_{1}$. We now claim that $f(M) \subset \bar{H}_{1}$. Let $M_{+}$and $M_{-}$be the two open half-hyperplanes into which $L$ divides $M$, so that $M=M_{+} \cup M_{-} \cup(M \cap L)$. Note that $f^{-1}\left(D_{1}\right)$ intersects both $M_{+}$and $M_{-}$nontrivially. Hence, any point $x \in M \backslash(M \cap L)$ can be connected to a point in $f^{-1}\left(D_{1}\right)$ by a path $\gamma \subset M$ which does not cross $M \cap L$. But then $f(\gamma)$ is a path connecting $f(x)$ to a point in $f(M) \cap D_{0} \subset \bar{H}_{1}$. Since $f$ is one-to-one, this path does not cross $f(L)$, hence $f(x) \in \bar{H}_{1}$.

Lemma 3.10 (No turns). Suppose $q$ is a (continuous) $(\kappa, C)$ quasi-isometry from a subset of $\mathbb{R}^{n}$ to a subset of $\mathbb{R}^{n}$. Suppose $B^{\prime}$ is a simply connected open subset of Euclidean space (with the Euclidean metric), contained in the image of $q$, and let $B=q^{-1}\left(B^{\prime}\right)$. Let $S_{1}, \ldots, S_{r}$ be $r$ fixed hyperplanes passing through the origin. We call any hyperplane parallel to one of the $S_{i}$ a "marked" hyperplane. Suppose there exist constants $m$ and $C^{\prime}>C$ such that for every marked hyperplane $L$ in $B$ there exist $m$ marked hyperplanes $L_{1}^{\prime}, \ldots, L_{m}^{\prime}$ in $B^{\prime}$ such that

$$
q(L) \subset \operatorname{Nbhd}\left(L_{1}^{\prime} \cup \cdots \cup L_{m}^{\prime}, C^{\prime}\right) .
$$

Then there exists $\beta=\beta^{\prime}(\kappa, X) C^{\prime}$ such that for every $L$ intersecting $B$, and every subset $B_{1}^{\prime} \subset B$ satisfying $d\left(B_{1}^{\prime}, \partial B\right) \gg \beta, q(L) \cap B_{1}^{\prime}$ is within $\beta$ of a single marked hyperplane $L^{\prime}$ in $B^{\prime}$. Conversely, for every marked hyperplane $L^{\prime}$ intersecting $B_{1}^{\prime}$ there exists a hyperplane $L$ in $B$ such that $q^{-1}\left(L^{\prime} \cap B_{1}^{\prime}\right)$ is within $\beta$ of $L$.

Proof. Since we will consider only marked hyperplanes, we will call them simply hyperplanes. Let $r$ denote the number of hyperplanes passing through a point, and let $s=2^{m} r$. Pick constants $\eta_{j}^{\prime}$ and $\lambda_{j k}^{\prime}, 0 \leq j \leq s, 0 \leq k \leq s$, such that 
$1=\eta_{0}^{\prime} \ll \cdots \ll \eta_{s-1}^{\prime} \ll \eta_{s}^{\prime}=\beta^{\prime}$, and $\eta_{j}^{\prime}=\lambda_{j 0}^{\prime} \ll \lambda_{j 1}^{\prime} \ll \cdots \ll \lambda_{j s}^{\prime}=\eta_{j+1}^{\prime}$. Write $\eta_{j}=\eta_{j}^{\prime} C^{\prime}$, and $\lambda_{j k}=\lambda_{j k}^{\prime} C^{\prime}$. Suppose there is a hyperplane $L$ for which the conclusion of the lemma is false with the choice of $\beta=\eta_{s}$.

For each $j$ suppose $L_{1}, \ldots, L_{b}$ are hyperplanes such that

$$
q(L) \subset \operatorname{Nbhd}\left(\bigcup_{k=1}^{b} L_{k}, \eta_{j}\right) .
$$

The union of the $L_{k}$ has the structure of a cellular complex $Y_{j}$ in $\mathbb{R}^{n}$ of dimension $n-1$; we shall refer to cells of dimension $n-1$ as simply "cells". We may compose $q$ with nearest point projection onto $Y_{j}$ to get a $\left(\kappa, \eta_{j}\right)$ quasi-isometry $q_{j}: L \rightarrow Y_{j}$. Using the standard "connect-the-dots" construction, we may assume that $q_{j}$ is continuous.

By Lemma A.3 (applied to $q$ composed with the nearest point projection onto $Y_{j}$ ) there exists a parameter $\rho_{j}, \eta_{j} \ll \rho_{j} \ll \eta_{j+1}$, such that

$$
h d\left(q(L) \cap B_{1}^{\prime}, \bigcup_{Q \in \mathcal{I}} Q \cap B_{1}^{\prime}\right) \leq \rho_{j}
$$

where $\mathcal{I}$ is a certain subset of the cells of $Y_{j}$. We define $d\left(Y_{j}\right)$ to be the minimum possible cardinality of the subset $\mathcal{I}$, such that $(26)$ holds (with fixed $\rho_{j}$ ). Then clearly $d\left(Y_{j}\right)$ is less than the total number of cells, which is bounded by $2^{b}$. Let $d_{j}$ be the minimum of $d\left(Y_{j}\right)$ over all possible $Y_{j}$. Note that $d_{j+1} \leq d_{j}$. Since $d_{1} \leq 2^{m}$ and $s>2^{m}$, we claim that there exists $j$ such that $d_{j}=d_{j+1}$.

Now fix such a $j$, and let $d=d_{j}=d_{j+1}$. Let $Q_{1}^{\prime}, \ldots, Q_{d}^{\prime}$ denote the cells in $\mathcal{I}$. We claim that there exists a point $p$ on $q(L)$ such that within $\eta_{j}$ of $p$ there exists at least two cells, say $Q_{1}^{\prime}$ and $Q_{2}^{\prime}$, which do not lie on the same hyperplane (indeed if this was false, $q(L)$ would be within $\eta_{j}$ of one hyperplane). The cells $Q_{1}^{\prime}$ and $Q_{2}^{\prime}$ have diameter at least $\eta_{j+1}-\eta_{j}$ (otherwise $d_{j+1}<d_{j}$ ).

Let $L_{1}, \ldots, L_{r}$ be the hyperplanes passing through $q^{-1}(p)$. By assumption $q\left(L_{j}\right) \cap B^{\prime}$ is within the $C^{\prime}$ neighborhood of at most $m$ hyperplanes $S_{j 1}, \ldots, S_{j m}$. Denote by $L_{j k}$ the hyperplanes parallel to $S_{j k}$ so that all these hyperplanes pass through $p$.

If the hyperplane $S_{j i}$ intersects $B\left(p, \lambda_{j k}\right)$, then the $C^{\prime}$-neighborhood of $S_{j i}$ will be within the $2 \lambda_{j k}$-neighborhood of $L_{j i}$ since $\lambda_{j k} \gg C^{\prime}$. Consider the ball $B\left(p, \lambda_{j 1}\right)$. If not all hyperplanes $S_{j i}$ intersecting $B\left(p, \lambda_{j 1}\right)$ intersect $B\left(p, \lambda_{j 0}\right)$, then we consider $B\left(p, \lambda_{j 2}\right)$. Again if not all hyperplanes intersecting $B\left(p, \lambda_{j 2}\right)$ intersect $B\left(p, \lambda_{j 1}\right)$, then consider $B\left(p, \lambda_{j 3}\right)$ and etc. Doing this for each $1 \leq l \leq r$ and since $s>m r$ we get that there exists an integer $k$ such that for each $l, 1 \leq l \leq r, q\left(L_{l}\right) \cap B\left(p, \lambda_{j, k+1}\right)$ is within a distance $2 \lambda_{j k}$ of a union of hyperplanes passing through $p$.

We now compose $q$ with nearest-point projection to get a map (which we call $\hat{q}$ ) from $Y=\bigcup_{i=1}^{r} L_{i} \cap B\left(q^{-1}(p), \lambda_{j, k+1}\right)$ to $Y^{\prime}=\bigcup_{i=1}^{r} L_{i}^{\prime} \cap B\left(p, \lambda_{j, k+1}\right)$, where the $L_{i}^{\prime}$ are the hyperplanes passing through $p$. Note that $\hat{q}$ is a $\left(\kappa, 2 \lambda_{j k}\right)$ quasi-isometry.

Denote by $Z$ the $n-2$-skeleton of $Y$, i.e. the union of all intersections $L_{i} \cap L_{m}, 1 \leq$ $i, m \leq r$, and by $Z^{\prime}$ the union of all intersections $L_{i}^{\prime} \cap L_{m}^{\prime}, 1 \leq i, m \leq r$. Let $B_{p}^{\prime}$ denote the ball $B\left(p, \frac{1}{2} \lambda_{j, k+1}\right)$ and $B_{p}=B\left(q^{-1}(p), \frac{1}{2} \lambda_{j, k+1}\right)$. By (26), for each hyperplane $L_{i}$ there exists a union of cells $\mathcal{D}_{i} \subset Y^{\prime}$ such that $h d\left(q\left(L_{i}\right) \cap B_{p}^{\prime}, \mathcal{D}_{i}\right) \leq$ $\lambda^{(4)}$ where $\lambda_{j k} \ll \lambda^{(4)} \ll \lambda_{j, k+1}$. Note that since $\hat{q}$ is a $\left(\kappa, 2 \lambda_{j k}\right)$ quasi-isometric embedding, and the diameters of the cells in $Y^{\prime}$ are $\succ \lambda_{j, k+1} \gg \lambda^{(4)}$, for $i \neq m$ the 
cells in $\mathcal{D}_{i}$ and $\mathcal{D}_{m}$ are distinct. Thus, $\mathcal{D}_{i} \cap \mathcal{D}_{m} \subset Z^{\prime}$. Applying Lemma A.4, part (ii), with $U=L_{i} \cap \hat{q}^{-1}\left(B_{p}^{\prime}\right), V=L_{m} \cap \hat{q}^{-1}\left(B_{p}^{\prime}\right), U^{\prime}=L_{i} \cap B_{p}^{\prime}, V^{\prime}=L_{m} \cap B_{p}^{\prime}$, and letting $i, m$ vary, we get that there exists a constant $\lambda^{(3)}$, with $\lambda^{(4)} \ll \lambda^{(3)} \ll \lambda_{j, k+1}$, such that

$$
\hat{q}(Z) \cap B_{p}^{\prime} \subset \operatorname{Nbhd}\left(Z^{\prime} \cap B_{p}^{\prime}, \lambda^{(3)}\right) .
$$

Let $Q$ be any cell of $Y$. By a volume preservation argument, there exists a cell $Q^{\prime}$ of $Y^{\prime}$ such that $\hat{q}(Q)$ intersects $\operatorname{Int}\left(Q^{\prime}, \lambda^{(2)}\right)$, where $\lambda^{(3)} \ll \lambda^{(2)} \ll \lambda_{j, k+1}$. In view of $(27)$ and the fact that $\hat{q}$ is a $\left(\kappa, 2 \lambda_{j k}\right)$ quasi-isometric embedding,

$$
\hat{q}^{-1}\left(\operatorname{Int}\left(Q^{\prime}, \lambda^{(2)}\right) \cap B_{p}^{\prime}\right) \subset \operatorname{Int}\left(Q, \lambda^{(3)}\right) .
$$

Thus, we may apply Corollary A.2 with $U=Q$ and $V=Q^{\prime}$ to show that

$$
\operatorname{Int}\left(Q^{\prime}, \lambda^{(2)}\right) \cap B_{p}^{\prime} \subset \operatorname{Nbhd}\left(q(Q), \lambda^{(1)}\right) \cap B_{p}^{\prime},
$$

where $\lambda^{(2)} \ll \lambda^{(1)} \ll \lambda_{j, k+1}$. Since $\hat{q}$ is a quasi-isometric embedding, the images of distinct cells must consist of distinct cells. Hence, since the number of cells in $Y^{\prime}$ is equal to the number of cells in $Y$, for any cell $Q_{i} \in Y$ there exists a unique cell $Q_{i}^{\prime} \in Y^{\prime}$ such that $h d\left(\hat{q}\left(Q_{i}\right) \cap B_{p}^{\prime}, Q_{i}^{\prime} \cap B_{p}^{\prime}\right) \leq \lambda^{(1)}$. The adjacency relations are preserved since by Lemma A.4, part (ii), for any cells $Q_{l}, Q_{m}$ of $Y$, $h d\left(q\left(Q_{l} \cap Q_{m}\right) \cap B_{p}^{\prime}, Q_{l}^{\prime} \cap Q_{m}^{\prime} \cap B_{p}^{\prime}\right) \leq \lambda^{(0)}$, where $\lambda^{(1)} \ll \lambda^{(0)} \ll \lambda_{j, k+1}$. Hence, by a volume preservation argument, $\operatorname{dim}\left(Q_{l} \cap Q_{m}\right)=\operatorname{dim}\left(Q_{l}^{\prime} \cap Q_{m}^{\prime}\right)$.

Thus $\hat{q}$ induces a one-to-one map of complexes $f: Y \rightarrow Y^{\prime}$. Since we know that $\hat{q}(L)$ is not contained in a single hyperplane, by Lemma 3.9, there exists a hyperplane $M \in Y$ such that $f(M)$ does not cross $f(L)$. Pick $x_{1}, x_{2} \in M$ on opposite sides of $L$ such that $\lambda^{(0)} \ll d\left(x_{i}, \hat{q}^{-1}(p)\right) \ll \lambda_{j, k+1}$, and $d\left(x_{i}, L\right) \gg \lambda^{(0)}$, $i=1,2$. Note that $q(x)$ and $\hat{q}(x)$ differ by $O\left(\lambda_{j k}\right) \ll \lambda^{(0)}$ on $Y$, and $q$ is onto $B_{p}^{\prime}$ by Lemma A.1. Then $d\left(q\left(x_{i}\right), f(L)\right) \gg \lambda^{(0)}$. Since $f(L)$ is a finite union of disjoint cells, and the points $\hat{q}\left(x_{1}\right) \in \hat{q}(M)$ and $\hat{q}\left(x_{2}\right) \in \hat{q}(M)$ are on the same side of $f(L)$, there exists a path $\gamma \subset B_{p}^{\prime}$ connecting $q\left(x_{1}\right)$ and $q\left(x_{2}\right)$ with $d(\gamma, f(L)) \gg \lambda^{(0)}$.

Then $\gamma_{0}=q^{-1}(\gamma) \subset q^{-1}\left(B_{p}^{\prime}\right)$ is a path connecting $x_{1}$ and $x_{2}$ with $d\left(\gamma_{0}, L\right) \gg$ $\lambda^{(0)}$. This is a contradiction since $x_{1}$ and $x_{2}$ are on opposite sides of $L$.

To prove the converse let $L^{\prime}$ be a hyperplane in $B_{1}^{\prime}$, and pick a point $x^{\prime} \in L^{\prime}$. Let $x=\phi^{-1}\left(x^{\prime}\right)$ and consider all planes $L_{1}, \ldots, L_{r}$ passing through $x$. As was proven above for each $q\left(L_{j}\right), 1 \leq j \leq r$, there exists $L_{j}^{\prime}$ such that $q\left(L_{j}\right) \cap B_{1}^{\prime} \subset$ $\operatorname{Nbhd}\left(L_{j}^{\prime}, \beta\right) \cap B_{1}^{\prime}$. Since $x^{\prime} \in q\left(L_{j}\right)$ we can by taking a parallel hyperplane and changing $\beta$ to $2 \beta$ assume that $L_{j}^{\prime}$ passes through $x^{\prime}$. Since $q$ is $(\kappa, 2 \beta)$ quasi-isometry we have that distinct $L_{j}$ correspond to distinct $L_{j}^{\prime}$. Therefore there is an $L$ such that $q(L) \cap B_{1}^{\prime} \subset \operatorname{Nbhd}\left(L^{\prime}, 2 \beta\right) \cap B_{1}^{\prime}$. Modifying $q$ on $L$ by composing it with the nearest point projection on $L^{\prime}$ and using Lemma A.1, we get $L^{\prime} \cap B_{1}^{\prime} \subset \operatorname{Nbhd}\left(q(L), \beta^{\prime}\right)$. Therefore, since $q$ is a quasi-isometry, $q^{-1}\left(L^{\prime} \cap B_{1}^{\prime}\right) \subset \operatorname{Nbhd}\left(L, \beta_{1}\right)$.

\subsection{Hyperplanes and Weyl chambers.}

Lemma $3.11(\tilde{\phi}(F)$ is close to cone over limit set). Suppose $F \in \mathcal{F}$ is a flat, and let $\tilde{\phi}$ be the graded quasi-isometric embedding of Step 2. Then,

$$
\tilde{\phi}(F) \sim_{\lambda \epsilon} \mathcal{V}
$$


where $\lambda$ is a constant depending only on $\kappa$ and $X$, and $\mathcal{V}$ is the union of Weyl chambers passing through the origin:

$$
\mathcal{V}=\bigcup_{k \in \mathcal{L}(\phi)} \pi\left(k A_{+}\right) \subset X
$$

where the limit set $\mathcal{L}(\phi)$ is defined in [EF, Definition 5.1].

Note. Unlike Lemma 3.6 we do not claim a bound of the type $\tilde{\phi}(F) \sim_{\lambda \epsilon, \phi(x), \lambda \rho} \mathcal{V}$.

Proof. Argue as in the proof of [EF, Lemma 8.3].

Lemma 3.12 (Walls into hyperplanes). Suppose $F \subset \mathcal{F}^{*}$ is a flat, and let $\mathcal{V}$ be as in Lemma 3.11. Let $L^{\prime}$ be a codimension 1 wall such that outside a sufficiently large ball, $L^{\prime}$ is contained completely in a single chamber $\mathfrak{C}^{\prime} \subset \mathcal{V}$. Then there exists a hyperplane $L \subset F$ such that, outside a sufficiently large ball, $L^{\prime} \subset \tilde{\phi}(L)[\lambda \epsilon]$ for some constant $\lambda$ depending only on $\kappa$ and $X$.

Conversely, if $L$ is a wall in $F$ such that outside a sufficiently large ball $\tilde{\phi}(L)$ is contained completely in $\mathfrak{C}^{\prime}[\lambda \epsilon]$ where $\mathfrak{C}^{\prime}$ is a chamber $\mathfrak{C}^{\prime}$ in $\mathcal{V}$, then there exists a hyperplane $L^{\prime}$ such that outside a sufficiently large ball, $\tilde{\phi}(L) \cap \mathfrak{C}^{\prime} \subset L^{\prime}[\lambda \epsilon]$.

Proof. Let $A^{\prime}\left(R_{j}, 4 R_{j}\right)$ be an annular domain in the chamber $\mathfrak{C}^{\prime}=\pi\left(k A_{+}\right)$centered at $e$, where $R_{j}=2^{j} R_{1}$ with $R_{1}=2 \kappa R$. Denote by $B_{j}^{\prime}$ the part of $\mathfrak{C}^{\prime} \cap A^{\prime}\left(R_{j}, 4 R_{j}\right)$ such that

$$
d\left(B_{j}^{\prime}, \partial \mathfrak{C}^{\prime}\right) \gg \beta^{\prime}(\kappa, X) C^{\prime}
$$

where $\beta^{\prime}(\kappa, X)$ and $C^{\prime}=O\left(\epsilon R_{j}\right)$ are the same as in Lemma 3.5 and Lemma 3.10 and let $B_{j}=\tilde{\phi}^{-1}\left(B_{j}^{\prime}\right)$. Let $L^{\prime}$ be a codimension 1 wall contained in $\mathfrak{C}^{\prime}$. Denote by $L_{j}^{\prime}$ a parallel translate of $L^{\prime}$ such that $L_{j}^{\prime} \cap B_{j}^{\prime}$ is not empty. Note that we can choose $L_{j}^{\prime}$ so that $d\left(L_{j}^{\prime} \cap A^{\prime}\left(R_{j}, 4 R_{j}\right), \partial \mathfrak{C}^{\prime}\right) \gg \beta^{\prime} \epsilon R_{j}$, and $d\left(L^{\prime}, L_{j}^{\prime}\right) \prec \epsilon R_{j}$. Combining Lemma 3.5 with the "converse" statement of Lemma 3.10 (with $B_{1}=B_{j}$ and $B_{1}^{\prime}=B_{j}^{\prime}$ defined as above) we get a hyperplane $L_{j} \subset F$ such that

$$
\tilde{\phi}\left(L_{j} \cap B_{j}\right) \subset L_{j}^{\prime}[\lambda \epsilon] \cap B_{j}^{\prime} \subset L^{\prime}\left[\lambda^{\prime} \epsilon\right] \cap B_{j}^{\prime}
$$

where $\lambda^{\prime}$ is independent of $\epsilon$ and $R_{j}$. Then by the local packing lemma (Lemma A.1),

$$
\tilde{\phi}\left(L_{j} \cap B_{j}\right)\left[\lambda^{\prime \prime} \epsilon\right] \supset L_{j}^{\prime} \cap B_{j}^{\prime} .
$$

Let $U_{j}^{\prime}$ be a ball in $L_{j}^{\prime} \cap A^{\prime}\left(2 R_{j}, 3 R_{j}\right)$ of radius $\succ R_{j}$. Since $L_{j}^{\prime}$ and $L_{j+1}^{\prime}$ are parallel there exists a ball $U_{j+1}^{\prime} \in L_{j+1}^{\prime}$ such that $h d\left(U_{j}^{\prime}, U_{j+1}^{\prime}\right)=O\left(\epsilon R_{j}\right)$. By (28) there exist subsets $U_{j} \in B_{j} \cap L_{j}$ and $U_{j+1} \in B_{j} \cap L_{j+1}$ so that $h d\left(\tilde{\phi}\left(U_{j}\right), U_{j}^{\prime}\right)=$ $O\left(\epsilon R_{j}\right)$ and $h d\left(\tilde{\phi}\left(U_{j+1}\right), U_{j+1}^{\prime}\right)=O\left(\epsilon R_{j}\right)$. Thus, $U_{j} \subset L_{j}, U_{j+1} \subset L_{j+1}$ and $h d\left(U_{j}, U_{j+1}\right)=O\left(\epsilon R_{j}\right)$. Also, since $\tilde{\phi}$ is a quasi-isometric embedding, $U_{j}$ contains a ball of radius $\succ R_{j}$. This implies that $L_{j}$ and $L_{j+1}$ are parallel, and $d\left(L_{j}, L_{j+1}\right) \leq$ $\lambda \epsilon R_{j}$. Hence

$$
d\left(L_{0}, L_{j}\right) \leq \sum_{k=1}^{j} d\left(L_{k-1}, L_{k}\right) \leq \sum_{k=1}^{j} \lambda \epsilon 2^{k} R_{1} \leq 2 \lambda \epsilon R_{j}
$$

and

$$
\tilde{\phi}\left(L_{0}\right) \cap B_{j}^{\prime} \subset \tilde{\phi}\left(L_{j}\right)\left[\lambda_{1} \epsilon\right] \cap B_{j}^{\prime} \subset L^{\prime}\left[\lambda_{2} \epsilon\right]
$$


Analogously, let $L$ be a wall in $F$ such that $\tilde{\phi}(L) \subset \mathfrak{C}^{\prime}[\lambda \epsilon]$. Let $A_{j}=A\left(R_{j}, 4 R_{j}\right)$ denote an annular domain in $F$ centered at $\pi(x)$. Let $B_{j} \subset A_{j}$ denote the part of $A_{j}$ such that $\tilde{\phi}\left(B_{j}\right) \subset \mathfrak{C}^{\prime}$ and $d\left(\tilde{\phi}\left(B_{j}\right), \partial \mathfrak{C}^{\prime}\right) \gg \beta^{\prime}(\kappa, X) C$. Let $B_{j}^{\prime}=\tilde{\phi}\left(B_{j}\right)$.

Denote by $L_{j}$ a hyperplane parallel to $L$ such that $d\left(L, L_{j}\right) \prec \epsilon R_{j}$ and $L_{j} \cap B_{j}$ is not empty. By Lemma 3.5 and Lemma 3.10 there exists a hyperplane $L_{j}^{\prime}$ such that $\tilde{\phi}\left(L_{j} \cap B_{j}\right) \subset L_{j}^{\prime}[\lambda \epsilon] \cap B_{j}^{\prime}$. Since $L_{j} \cap B_{j} \cap B_{j+1}$ is not empty and contains balls of radius $\succ R_{j}$ we get as above that $L_{j}^{\prime}$ and $L_{j+1}^{\prime}$ are parallel hyperplanes and $d\left(L_{j}^{\prime}, L_{j+1}^{\prime}\right)=O\left(\epsilon R_{j}\right)$. Therefore $d\left(L_{0}^{\prime}, L_{j}^{\prime}\right) \prec \epsilon R_{j}$ and

$$
\tilde{\phi}\left(L \cap A_{j}\right) \subset \tilde{\phi}\left(L_{j} \cap B_{j}\right)[\lambda \epsilon] \subset L_{j}^{\prime}\left[\lambda^{\prime} \epsilon\right] \cap B_{j}^{\prime} \subset L_{0}^{\prime}\left[\lambda^{\prime \prime} \epsilon\right] \cap B_{j}^{\prime} \subset L_{0}^{\prime}\left[\lambda^{\prime \prime} \epsilon\right] .
$$

Since $j$ is arbitrary we have $\tilde{\phi}(L) \cap \mathfrak{C}^{\prime} \subset L_{0}^{\prime}\left[\lambda^{\prime \prime} \epsilon\right]$.

Using Lemma 3.8 instead of Lemma 3.5 in the above proof we get the following lemma:

Lemma 3.13 (Hyperplanes to hyperplanes). Let $F \subset \mathcal{F}^{*}$ be a flat and suppose $\tilde{\phi}(F)$ is close (in the sense of Step $2(c)$ ) to a single flat $F^{\prime}$. Suppose $x \in \Omega_{F}^{*}$, and let $L \supset F$ be a hyperplane passing through $x$. Then, there exists a constant $\lambda$ depending only on $\kappa$ and $X$ and a hyperplane $L^{\prime} \subset F^{\prime}$ such that

$$
\tilde{\phi}(L) \sim_{\lambda \epsilon, \phi(x), \lambda \rho} L^{\prime} .
$$

Lemma 3.14 (Chambers to chambers). Suppose $\mathcal{F} \subset \mathcal{F}^{*}$, and let $\mathcal{V}$ be as in Lemma 3.11. Let $\mathfrak{C}$ be a chamber in $F$. Then there exists a chamber $\mathfrak{C}^{\prime}$ in $\mathcal{V}$ such that $\tilde{\phi}(\mathfrak{C}) \sim_{\lambda \epsilon} \mathfrak{C}^{\prime}$. The chamber $\mathfrak{C}^{\prime}$ is unique up to equivalence.

Proof. Let $\mathfrak{C}^{\prime}$ be an arbitrary chamber in $\mathcal{V}$. Taking a parallel translate of $\mathfrak{C}^{\prime}$ in $\mathcal{V}$ (and denoting it again by $\mathfrak{C}^{\prime}$ ) we can assume that $\tilde{\phi}$ packs $\mathfrak{C}^{\prime}$, i.e. $\tilde{\phi}(F) \supset \mathfrak{C}^{\prime} \backslash \partial \mathfrak{C}^{\prime}[\lambda \epsilon]$ (see [EF, Lemma 8.3] or Corollary A.2). By Lemma 3.12, for each wall $L_{i}^{\prime}$ in $\partial \mathfrak{C}^{\prime}$ there exists a hyperplane $L_{i}$ in $F$ such that outside a sufficiently large ball, $L_{i}^{\prime} \subset \tilde{\phi}\left(L_{i}\right)[\lambda \epsilon]$. By replacing $L_{i}$ by a parallel translate, we may assume that all $L_{i}$ pass through the point $x \in F$, and still, outside a sufficiently large ball, $L_{i}^{\prime} \subset \tilde{\phi}\left(L_{i}\right)[\lambda \epsilon]$. Let $\mathcal{P}_{j}$ be the polyhedra bounded by the $L_{i}$. By Corollary A.2 we may assume that (outside a sufficiently large ball), $\tilde{\phi}^{-1}\left(\mathfrak{C}^{\prime}\right)$ packs one of these polyhedra, which we denote $\mathcal{P}$, i.e. $\tilde{\phi}^{-1}\left(\mathfrak{C}^{\prime}\right) \supset \mathcal{P} \backslash \partial \mathcal{P}[\lambda \epsilon]$. Since the diameter of $\mathfrak{C}^{\prime} \cap A(R, 2 R)$ is $\succ R$, and $h d\left(\tilde{\phi}^{-1}\left(\mathfrak{C}^{\prime}\right) \cap A(R, 2 R), \mathcal{P} \cap A(R, 2 R)\right)=O(\epsilon R)$ (recall $\epsilon \ll 1), \mathcal{P}$ must contain a Weyl chamber $\mathfrak{C}$. Hence $\tilde{\phi}(\mathfrak{C}) \subset \tilde{\phi}(\mathcal{P}) \subset \mathfrak{C}^{\prime}[\lambda \epsilon]$.

Now consider a wall $L_{i}$ bounding $\mathfrak{C}$. Since $\tilde{\phi}\left(L_{i}\right) \subset \mathfrak{C}^{\prime}[\lambda \epsilon]$, arguing as in the proof of Lemma 3.12, we may conclude that $\tilde{\phi}\left(L_{i}\right)$ is contained in $L_{i}^{\prime}[\lambda \epsilon]$ for some hyperplane $L_{i}^{\prime}$ belonging to a flat $F^{\prime} \supset \mathfrak{C}^{\prime}$. We may replace the $L_{i}^{\prime}$ by parallel translates all passing through the same point; still, outside a sufficiently large ball, $\tilde{\phi}\left(L_{i}\right) \subset L_{i}^{\prime}[\lambda \epsilon]$. By the local packing lemma, $\tilde{\phi}$ restricted to $\mathfrak{C}[\lambda \epsilon]$ packs one of the polyhedra bounded by the $L_{i}^{\prime}$, say $\mathcal{P}^{\prime}$. Since $\tilde{\phi}(\mathfrak{C}) \subset \mathfrak{C}^{\prime}[\lambda \epsilon], L_{i}^{\prime} \subset \mathfrak{C}^{\prime}[2 \lambda \epsilon]$, hence $\mathcal{P}^{\prime} \subset \mathfrak{C}^{\prime}\left[\lambda^{\prime} \epsilon\right]$. Also since the injectivity radius of $\mathcal{P}^{\prime} \cap A(R, 2 R) \succ R, \mathcal{P}^{\prime}$ contains a Weyl chamber $\mathfrak{C}_{1}^{\prime} \subset \mathfrak{C}^{\prime}[\lambda \epsilon]$. By Lemma B.7 this can only happen if $\mathfrak{C}_{1}^{\prime \prime}$ is equivalent to $\mathfrak{C}^{\prime}$. Thus, outside of a large ball, $\mathfrak{C}^{\prime} \subset \tilde{\phi}(\mathfrak{C})[\lambda \epsilon]$.

Since $\tilde{\phi}^{-1}\left(\mathfrak{C}^{\prime}\right) \supset \mathcal{P} \backslash \partial \mathcal{P}[\lambda \epsilon]$ we have $\tilde{\phi}^{-1}\left(\mathfrak{C}^{\prime}\right)[\lambda \epsilon] \supset \mathcal{P} \supset \mathfrak{C}$. The uniqueness of $\mathfrak{C}$ follows from the last assertion of Lemma B.7.

Therefore we have a map from chambers in $\mathcal{V}$ to equivalence classes of chambers in $F$. This map is one-to-one and onto: the first property follows immediately from 
the fact that $\tilde{\phi}^{-1}$ is a graded quasi-isometry, and the second from the fact that $F \subset \tilde{\phi}^{-1}(\mathcal{V})[\lambda \epsilon]$ outside of a large ball.

Proof of Proposition 3.3. By Lemma 3.14 one has a well-defined map $\phi_{0}$ from the equivalence classes of chambers in $F$ (i.e. $F(\infty) \subset \hat{X}$ ) to equivalence classes of chambers in $\mathcal{V}$, i.e. $\mathcal{L}(\phi) \subset \hat{X}$.

From the proof of Lemma 3.14 it is clear that neighboring chambers in $F$ map to neighboring chambers (here neighboring means sharing a codimension 1 wall). This implies that if $\mathfrak{C}_{1}, \mathfrak{C}_{2}$ are chambers in $F$, and $k_{1}, k_{2}$ the corresponding points in $F(\infty)$, then $d_{c}\left(\phi_{0}\left(k_{1}\right), \phi_{0}\left(k_{2}\right)\right) \leq d_{c}\left(k_{1}, k_{2}\right)$. (Recall $d_{c}$ is the combinatorial distance in the Tits building $\mathcal{T}(X)$, i.e. the length of the minimal gallery connecting the two chambers.) Indeed there exists a minimal gallery $\gamma$ connecting $k_{1}$ and $k_{2}$ and lying in $F$; then $\phi_{0}(\gamma)$ is a gallery connecting $\phi_{0}\left(k_{1}\right)$ and $\phi_{0}\left(k_{2}\right)$ and $\ell\left(\phi_{0}(\gamma)\right) \leq \ell(\gamma)$.

From Lemma 3.11, and the proof of [EF, Theorem 1.1] (see [EF, §7]) there exist two limit points $a^{\prime}, b^{\prime} \in \mathcal{L}(\phi)$ such that $a^{\prime} \notin b^{\prime} \mathcal{S}$, i.e. $d_{c}\left(a^{\prime}, b^{\prime}\right)$ is maximal. Let $F^{\prime}$ denote the unique flat such that $a^{\prime}, b^{\prime} \subset F^{\prime}(\infty)$ (see [EF, §7.1]). Let $a, b$ be points in $F(\infty)$ such that $\phi_{0}(a)=a^{\prime}$ and $\phi_{0}(b)=b^{\prime}$. Then $d_{c}(a, b) \geq d_{c}\left(a^{\prime}, b^{\prime}\right)$, and since the latter is maximal, $d_{c}(a, b)$ is maximal. Let $\gamma$ denote any minimal gallery connecting $a$ and $b$; then $\phi_{0}(\gamma)$ is a minimal gallery connecting $a^{\prime}$ and $b^{\prime}$; since $F^{\prime}(\infty)$ is an apartment and apartments are convex (see [B, Proposition, §IV.4, p. 88]), $\phi(\gamma) \subset F^{\prime}(\infty)$. Since any chamber is contained in such a minimal gallery (see [B, Lemma, §IV.5, p. 92]), this implies that $\phi_{0}(F(\infty)) \subset F^{\prime}(\infty)$, i.e. $\mathcal{L}(\phi) \subset F^{\prime}(\infty)$. Now part (i) follows easily from Theorem 1.5 and [EF, Corollary 7.4].

Part (ii) follows immediately from combining part (i) with Lemma 3.14.

Proof of Proposition 3.4. Part (a) is immediate from Proposition 3.3 and Lemma 3.13. To get part (b) one may argue as follows: Let $Y \subset F$ denote the union of the hyperplanes passing though $x$, let $x^{\prime} \in F^{\prime}$ denote the nearest point in $F^{\prime}$ to $\phi(x)$, and let $Y^{\prime} \subset F^{\prime}$ denote the union of the hyperplanes passing through $x^{\prime}$. By part (a), $\tilde{\phi}(Y) \sim_{\lambda \epsilon, x^{\prime}, \lambda \rho} Y^{\prime}$. Note that $F \backslash Y$ is the union of the interiors of the chambers passing through $x$. For any chamber $\mathfrak{C} \subset F$ based at $x$ we may pick a point $u \in \mathfrak{C}$ such that $d(u, x) \gg \rho$ and $d(u, Y) \succ d(u, x)$. Then $d\left(\tilde{\phi}(u), Y^{\prime}\right) \succ d\left(\tilde{\phi}(u), x^{\prime}\right)$. Now part (b) follows from the graded version of Corollary A.2.

\section{THE BOUNDARY MAP}

Definition 4.1 (The boundary map $\phi_{0}: \mathcal{U} \rightarrow \hat{X}$ ). Let $\mathcal{U} \subset \hat{X}$ denote the set of equivalence classes of Weyl chambers of flats in $\mathcal{F}^{*}$. Let $\phi_{0}: \mathcal{U} \rightarrow \hat{X}$ be the map defined on these Weyl chambers by part (ii) of Step 3 .

We note that $\phi_{0}$ is well defined. Indeed, let $\mathfrak{C}_{1} \subset F_{1}$ and $\mathfrak{C}_{2} \subset F_{2}$ be equivalent Weyl chambers, i.e. $h d\left(\mathfrak{C}_{1}, \mathfrak{C}_{2}\right)<c<\infty$, and the flats $F_{1}$ and $F_{2}$ belong to $\mathcal{F}^{*}$. Pick $x_{0} \in F_{1}$. By Step 2, part (c), there exists a $\left(\kappa, \lambda \epsilon, C^{\prime}\right)$ graded q.i. embedding $\tilde{\phi}$ based at $x_{0}$ from $F_{1} \cup F_{2}$ to $X$, and $\lambda$ depends only on $\kappa$ and $X$. For any $x_{1} \in \mathfrak{C}_{1}$ there exists $x_{2} \in \mathfrak{C}_{2}$ such that $d\left(x_{1}, x_{2}\right) \leq c$. Then since $\tilde{\phi}$ is a graded q.i. embedding, $d\left(\tilde{\phi}\left(x_{1}\right), \tilde{\phi}\left(x_{2}\right)\right) \prec d\left(x_{1}, x_{2}\right)+\epsilon d\left(x_{1}, x_{0}\right)+\epsilon d\left(x_{2}, x_{0}\right) \prec \epsilon d\left(\tilde{\phi}\left(x_{1}\right), \tilde{\phi}\left(x_{0}\right)\right)$, where we assumed that $d\left(x_{1}, x_{0}\right) \gg \max \left(c / \epsilon, C^{\prime}\right)$. Since $x_{1} \in \mathfrak{C}_{1}$ is arbitrary, this shows that $\tilde{\phi}\left(\mathfrak{C}_{1}\right) \subset \tilde{\phi}\left(\mathfrak{C}_{2}\right)[\lambda \epsilon]$, outside of a large ball. Analogously one has $\tilde{\phi}\left(\mathfrak{C}_{2}\right) \subset \tilde{\phi}\left(\mathfrak{C}_{1}\right)[\lambda \epsilon]$. Therefore, $\tilde{\phi}\left(\mathfrak{C}_{1}\right) \sim_{\lambda \epsilon} \tilde{\phi}\left(\mathfrak{C}_{2}\right)$. Hence if we denote $\phi_{0}\left(\mathfrak{C}_{i}\right)$ by $\mathfrak{C}_{i}^{\prime}$, 
then $\mathfrak{C}_{1}^{\prime} \sim_{\lambda \epsilon} \mathfrak{C}_{2}^{\prime}$. Then $\mathfrak{C}_{1}^{\prime \prime}$ is equivalent to $\mathfrak{C}_{2}^{\prime}$ by the last assertion of Lemma B.7. This shows that $\phi_{0}$ is well defined.

Recall that the cells of maximal dimension in the Tits building $\mathcal{T}(X)$ are equivalence classes of Weyl chambers, i.e. points in $K / M$. For $\sigma \subset \Delta$ and $k \in K, k K_{\sigma}$ can be viewed as a cell in $\mathcal{T}(X)$ of codimension $|\sigma|$ (here for a subset $\sigma$ of $\Delta,|\sigma|$ is the cardinality of $\sigma$ ).

Definition 4.2 (Tits map defined on a subset). Let $E$ be a subset of $\hat{X}$, and let $\psi: E \rightarrow \hat{X}$ be a map. We say that $\psi$ is a Tits map defined on $E$ if for all $k \in E$ and any $\sigma \subset \Delta$ there exists $\tau \subset \Delta$ with $|\tau|=|\sigma|$ such that $\psi\left(k K_{\sigma} \cap E\right) \subset \psi(k) K_{\tau}$.

In other words, on its domain of definition, $\psi$ sends adjacent simplices in $\mathcal{T}(X)$ into adjacent simplices, and preserves the dimension of the intersection. Note that we do not require $\psi$ to be continuous.

Step $4\left(\phi_{0}\right.$ preserves partial ordering in $\left.\mathcal{T}(X)\right)$. The map $\phi_{0}: \mathcal{U} \rightarrow \hat{X}$ defined in Definition 4.1 is a Tits map defined almost everywhere.

The proof uses the following:

Lemma 4.3 (Walls to walls). Suppose $F \in \mathcal{F}^{*}$ is a flat and let $F^{\prime}$ be as in Step 3. Let $L \subset F$ be a $\sigma$-wall. Then there exists a $\tau$-wall $L^{\prime}$ in $F^{\prime}$ such that $|\sigma|=|\tau|$, and $\tilde{\phi}(L) \sim_{\lambda \epsilon} L^{\prime}$. The constant $\lambda$ depends only on $\kappa$ and $X$.

Proof of Lemma 4.3. The wall $L$ may be viewed as the intersection of a collection of Weyl chambers $\mathfrak{C}_{1}, \ldots, \mathfrak{C}_{r} \subset F$. By Step 3 there exist Weyl chambers $\mathfrak{C}_{i}^{\prime} \subset F^{\prime}$ such that $\tilde{\phi}\left(\mathfrak{C}_{i}\right) \sim_{\lambda \epsilon} \mathfrak{C}_{i}^{\prime}$. We may assume all the $\mathfrak{C}_{i}^{\prime}$ are based at the same point $x^{\prime} \in F^{\prime}$ (otherwise replace them by equivalent Weyl chambers). Let $L^{\prime}=\bigcap_{i=1}^{r} \mathfrak{C}_{i}^{\prime}$. From the geometry of the root system (i.e. all angles bounded from below), it is clear that (the graded version of) Lemma A.4, part (ii), can be applied repeatedly. Thus, $\tilde{\phi}(L) \sim_{\lambda \epsilon} L^{\prime}$. The assertion that $|\sigma|=|\tau|$ follows easily by volume considerations, or the packing lemma (cf. Lemma A.1).

Proof of Step 4. Suppose $\mathfrak{C}_{1}, \mathfrak{C}_{2} \in \mathcal{U}$, and let $k_{i} \in K$ be such that $\pi\left(k_{i} A^{+}\right)$is equivalent to $\mathfrak{C}_{i}$ for $i=1,2$. By assumption $k_{2} \in k_{1} K_{\sigma}$. This implies that there exist $\sigma$-walls $L_{i} \subset \partial \mathfrak{C}_{i}$ such that $h d\left(L_{1}, L_{2}\right)<\infty$. Let $\mathfrak{C}_{i}^{\prime}=\phi_{0}\left(\mathfrak{C}_{i}\right)$.

By Lemma 4.3 there exists $\tau \subset \Delta$ with $|\tau|=|\sigma|$ and a $\tau$-wall $L_{1}^{\prime}$ such that $\tilde{\phi}\left(L_{1}\right) \sim_{\lambda \epsilon} L_{1}^{\prime}$. Since $L_{1}$ and $L_{2}$ are equivalent, this also implies that $\tilde{\phi}\left(L_{2}\right) \sim_{\lambda \epsilon} L_{1}^{\prime}$. Hence, outside a sufficiently large ball, $L_{1}^{\prime} \subset \mathfrak{C}_{1}^{\prime}\left[\lambda^{\prime} \epsilon\right] \cap \mathfrak{C}_{2}^{\prime}\left[\lambda^{\prime} \epsilon\right]$.

Let $k_{1}^{\prime}, k_{2}^{\prime}, k^{\prime} \in K$ be such that $h d\left(\pi\left(k_{i}^{\prime} A^{+}\right), \mathfrak{C}_{i}^{\prime}\right)<\infty$ and $h d\left(\pi\left(k^{\prime} A_{\tau}^{+}\right), L_{1}^{\prime}\right)<\infty$. Then, outside a sufficiently large ball, $\pi\left(k^{\prime} A_{\tau}^{+}\right) \subset \pi\left(k_{1}^{\prime} A^{+}\right)\left[\lambda^{\prime \prime} \epsilon\right] \cap \pi\left(k_{2}^{\prime} A^{+}\right)\left[\lambda^{\prime \prime} \epsilon\right]$. Then by Lemma B.7, $k_{i}^{\prime} \subset k^{\prime} K_{\tau}$.

\section{UNIFORM CONTINUITY OF THE BOUNDARY MAP}

Step 5 ( $\phi_{0}$ is bi-Hölder). There exists a set $\mathcal{U}_{2} \subset \mathcal{U}_{1} \subset K / M$ of full ( $K$-invariant) measure such that for $x, y \in \mathcal{U}_{2}$, the map $\phi_{0}$ satisfies the Hölder type inequalities

$$
c^{-1} d_{K}(x, y)^{\lambda} \leq d_{K}\left(\phi_{0}(x), \phi_{0}(y)\right) \leq c d_{K}(x, y)^{1 / \lambda}, \quad x, y \in \mathcal{U}_{2} .
$$

Here $d_{K}$ is the left $K$-invariant metric on $\hat{X} \cong K / M$, and $\lambda$ and $c$ do not depend on $x, y$. 
Remark. The identification of $\hat{X}$ with $K / M$ and thus $d_{K}$ depends on the choice of origin. However, since the different possible metrics are conjugate by diffeomorphisms and thus equivalent, the notion of bi-Hölder is independent of choices. For chambers $\mathfrak{C}_{1}, \mathfrak{C}_{2} \subset X$, we let $d_{K}\left(\mathfrak{C}_{1}, \mathfrak{C}_{2}\right)=d_{K}\left(\mathfrak{D}_{1}, \mathfrak{D}_{2}\right)$, where $\mathfrak{D}_{i}$ is equivalent to $\mathfrak{C}_{i}$ and based at $e$.

The motivation for Step 5 is the following well-known lemma:

Lemma 5.1. Let $Y$ be a rank 1 symmetric space, and let $q: Y \rightarrow Y$ be a quasiisometry. Then the induced boundary map $q_{0}: \hat{Y} \rightarrow \hat{Y}$ is bi-Holder. (Here $\hat{Y}$ is the boundary at infinity, i.e. the equivalence classes of geodesic rays.)

Proof of Lemma 5.1. Without loss of generality, $q(e)=e$. Let $\gamma_{1}=\pi\left(k_{1} A^{+}\right)$ and $\gamma_{2}=\pi\left(k_{2} A^{+}\right)$be geodesic rays starting at $e$, where $k_{1}, k_{2} \in K$. Write $\theta=d_{K}\left(k_{1}, k_{2}\right)$; then $\theta$ is the angle between $\gamma_{1}$ and $\gamma_{2}$. We parameterize the $\gamma_{i}$ by arc length. Pick a sufficiently large $Q>0$, and let $t=\sup \{s: s>$ 0 and $\left.d\left(\gamma_{1}(s), \gamma_{2}(s)\right)<Q\right\}$. By Lemma B.5, $c_{1}|\log \theta|<t<c_{2}|\log \theta|$ where $c_{1}, c_{2}$ are independent of $\theta$.

By Mostow's lemma, there exist geodesic rays $\gamma_{i}^{\prime}$ and a constant $N$ depending only on $Y$ and the q.i. constants of $q$ such that $h d\left(q\left(\gamma_{i}\right), \gamma_{i}^{\prime}\right)<N$. Without loss of generality, the $\gamma_{i}^{\prime}$ also start at $e$, and $Q \gg N$. Let $t^{\prime}=\sup \{s$ : $s>0$ and $\left.d\left(\gamma_{1}^{\prime}(s), \gamma_{2}^{\prime}(s)\right)<2 \kappa Q\right\}$ and $t^{\prime \prime}=\sup \left\{s: s>0\right.$ and $d\left(\gamma_{1}(s), \gamma_{2}(s)\right)<$ $Q /(2 \kappa)\}$. Since $q$ is a quasi-isometry, and $Q \gg N, t \prec t^{\prime \prime} \leq t^{\prime} \prec t$. Hence by Lemma B.5, $c_{1}^{\prime}\left|\log \theta^{\prime}\right| \leq|\log \theta| \leq c_{2}^{\prime}\left|\log \theta^{\prime}\right|$ where $c_{1}^{\prime}$ and $c_{2}^{\prime}$ are independent of $\theta$ and $\theta^{\prime}$ is the angle between $\gamma_{1}^{\prime}$ and $\gamma_{2}^{\prime}$. After exponentiating, we get the desired estimate.

The proof of Lemma 5.1 carries over easily to the higher rank case - cf. Lemma 5.4. However, the presence of holes may create difficulties, since the entire argument in the proof of Lemma 5.1 may take place in a hole, e.g. when trying to prove continuity across the endpoint of a horosphere. In the higher rank case, this difficulty can be overcome as follows:

Suppose we consider two Weyl chambers $\mathfrak{C}_{1}$ and $\mathfrak{C}_{2}$ based at $e$ which share a codimension 1 wall $L_{0}$. Let $F_{1}$ be a flat containing $\mathfrak{C}_{1}$ and $F_{2}$ a flat containing $\mathfrak{C}_{2}$; then $F_{1}$ and $F_{2}$ intersect in a hyperplane $L$ which contains $L_{0}$. Note that $F_{1}$ and $F_{2}$ are contained in a totally geodesic submanifold $Z$ of $X$ which is a metric product $Z=L \times Y$, where $Y$ is a rank 1 symmetric space. In fact, $F_{1} \cup F_{2}=L \times\left(\gamma_{1} \cup \gamma_{2}\right)$, where $\gamma_{1}$ and $\gamma_{2}$ are two geodesics in $Y$ passing through the same point. The angle $d_{K}\left(\mathfrak{C}_{1}, \mathfrak{C}_{2}\right)$ is the same as the angle between the geodesics $\gamma_{1}$ and $\gamma_{2}$ and can be measured above any point of $L$.

The strategy of the proof of Step 5 is the following: suppose the common base point of $\mathfrak{C}_{1}$ and $\mathfrak{C}_{2}$ is in or near a hole (in $F_{1}$ or $F_{2}$ ). By the Moore ergodicity theorem, applied to the subgroups $A_{\sigma}$ of $A$, we may assume that $L$ is uniformly distributed; hence there is a point $p$ of $L_{0}$ which is away from the holes in $F_{1}$ and $F_{2}$. We then apply the argument in the proof of Lemma 5.1 to $p \times\left(\gamma_{1} \cup \gamma_{2}\right) \subset p \times Y \cong Y$.

For this argument, we need results on images of hyperplanes similar to Step 3, part (i) (Proposition 3.4 (a) is not sufficient). This, together with some related issues is done in $\S 5.1$. The program sketched in the previous paragraph is carried out in $\S 5.3$. The assumption that $\mathfrak{C}_{1}$ and $\mathfrak{C}_{2}$ share a codimension 1 wall is removed in $\S 5.4$. 


\subsection{Mapping hyperplanes.}

Lemma 5.2 (Hyperplanes to hyperplanes, uniform version). There exists a constant $N=N(\kappa, \epsilon, C)$, a family of hyperplanes $\mathcal{L}$, and for each $L \in \mathcal{L}$ nonempty subsets $\Omega_{L} \subset L$, such that the following hold:

(a) $\mathcal{L}$ is $\Gamma$-invariant, i.e. if $\gamma \in \Gamma$ and $L \in \mathcal{L}$, then $\gamma L \in \mathcal{L}$.

(b) $\mathcal{L}$ has full measure, i.e. there exists a set $E \subset \Gamma \backslash G$ with $\mu(E)=1$ such that for any $y \in p^{-1}(E)$ and any $\alpha \in \Sigma, \pi\left(y A_{\alpha}\right) \in \mathcal{L}$.

(c) For every $L \in \mathcal{L}$, there exists a hyperplane $L^{\prime}$ such that $\phi\left(\Omega_{L}\right)$ lies within a distance $N$ of $L^{\prime}$.

(d) For every $L \in \mathcal{L}$, and every $x \in L$,

$$
\liminf _{r \rightarrow \infty} \frac{\left|B(x, r) \cap \Omega_{L}\right|}{|B(x, r) \cap L|} \geq 1-|\Sigma| \epsilon .
$$

(e) For every $y \in p^{-1}(E), \pi(y A) \in \mathcal{F}^{*}$ and for any $\alpha \in \Sigma$

$$
\liminf _{r \rightarrow \infty} \frac{\left|\Omega_{F} \cap B(y, r) \cap L_{\alpha}\right|}{\left|B(y, r) \cap L_{\alpha}\right|} \geq 1-|\Sigma| \epsilon .
$$

Proof. Let $\Omega^{*}$ and $E^{*}$ be as in the proof of Lemma 3.2. Let $\chi_{\Omega^{*}}$ be the characteristic function of $\Omega^{*}$. By the ergodic theorem, Theorem 1.4, there exists a set $E \subset E^{*} \subset$ $\Gamma \backslash G$ with $\mu(E)=1$ such that for all $x \in E$, and all $\alpha \in \Sigma$,

$$
\lim _{r \rightarrow \infty} \frac{1}{\left|B_{r}\right|} \int_{B_{r}} \chi_{\Omega^{*}}(x \exp v) d \nu_{\alpha}(v)=\mu\left(\Omega^{*}\right) \geq 1-|\Sigma| \epsilon
$$

where $\nu_{\alpha}$ is Lebesgue measure on the Lie algebra of $A_{\alpha} \cong \mathbb{R}^{n-1}$, and here $B_{r}$ is the ball of radius $r$ in this Lie algebra. Let $\mathcal{L}=\left\{\pi\left(y A_{\alpha}\right) \quad: \quad y \in p^{-1}(E)\right\}$. Then (a) and (b) hold by construction. For $L=\pi\left(z A_{\alpha}\right) \in \mathcal{L}$, let $\Omega_{z, L}=\{\pi(z \exp v)$ : $p(z \exp v) \in \Omega^{*}$ and $\left.v \in A_{\alpha}\right\}$, and $\Omega_{L}=\bigcup_{z \text { with } L=\pi\left(z A_{\alpha}\right)} \Omega_{z, L}$. Then, (d) holds (already for $\Omega_{z, L}$ ) by (30). Also (e) holds since $\Omega_{z, L} \subset \Omega_{F}$ and (30).

It remains to prove (c). In fact we will show that (c) holds already for $\Omega_{z, L} ; \Omega_{L}$ is introduced only to simplify notation.

Suppose $x \in \Omega_{z, L}$. Let $F_{1}=\pi(z A)$. Then $x \in \Omega_{F_{1}}$, and there exists $F_{2} \in$ Trans $_{F_{1}}(x)$ such that $F_{1} \cap F_{2}=L$ and $x \in \Omega_{F_{2}}$. Let $F_{1}^{\prime}, F_{2}^{\prime}$ be as in Step 3.

Since $\Omega_{z, L} \subset L \cap \Omega_{F_{1}} \cap \Omega_{F_{2}}$, by Step 3 (i), $\phi\left(\Omega_{z, L}\right) \subset \operatorname{Nbhd}\left(F_{1}^{\prime}, N\right) \cap \operatorname{Nbhd}\left(F_{2}^{\prime}, N\right)$. Choose $r \gg N$, and let $\mathcal{P}^{\prime}$ be as in Lemma B.1, part (i), re. the flats $F_{1}^{\prime}$ and $F_{2}^{\prime}$. Then,

$$
\phi\left(\Omega_{z, L}\right) \subset \operatorname{Nbhd}\left(\mathcal{P}^{\prime}, O(r)\right) .
$$

Let $\tilde{\phi}: F_{1} \cup F_{2} \rightarrow X$ be as in Step 2, part (c). Note that since (d) holds for $\Omega_{z, L}$, there exists $\lambda$ depending only on $X$ such that $\Omega_{z, L}[\lambda \epsilon]=L$. Then (31) implies that (outside a sufficiently large ball)

$$
\tilde{\phi}(L) \subset \mathcal{P}^{\prime}\left[\lambda^{\prime} \epsilon\right] .
$$

Conversely, choose any $x \in \mathcal{P}^{\prime}$ with $\epsilon d(x, z) \gg r$, and let $\tilde{\phi}^{*}: F_{1}^{\prime} \cup F_{2}^{\prime} \rightarrow F_{1} \cup F_{2}$ be the coarse inverse of $\tilde{\phi}$. Then, since $\tilde{\phi}^{*}$ is a graded quasi-isometry, $\tilde{\phi}^{*}(x)\left(F_{i}^{\prime}\right)=F_{i}$, and $x \in \operatorname{Nbhd}\left(F_{1}^{\prime}, O(r)\right) \cap \operatorname{Nbhd}\left(F_{2}^{\prime}, O(r)\right)$, we have $\tilde{\phi}^{*}(x) \in F_{1}\left[\lambda_{1} \epsilon\right] \cap F_{2}\left[\lambda_{1} \epsilon\right]$. Hence by Lemma 3.1, $\tilde{\phi}^{*}(x) \in L\left[\lambda_{1}^{\prime} \epsilon\right]$. Hence, combining with (32), we get $\tilde{\phi}(L) \sim_{\lambda^{\prime} \epsilon} \mathcal{P}^{\prime}$.

By Proposition 3.4, there exists a hyperplane $L^{\prime}$ such that $\tilde{\phi}(L) \sim_{\lambda \epsilon} L^{\prime}$. Hence $\mathcal{P}^{\prime} \sim_{\lambda^{\prime \prime} \epsilon} L^{\prime}$. This implies that $\mathcal{P}$ is a strip between two hyperplanes parallel to $L^{\prime}$. 
In view of (31), to show (c) we need only show that the width of the strip is $O(r)$. This can be checked as follows: Choose $R$ so that $r \ll R \ll r / \epsilon$. Then, by Lemma 3.7, part (a), the injectivity radius of $\mathcal{P}^{\prime} \cap B_{R}$ is $O(r)$. Since $\mathcal{P}^{\prime}$ is a strip, this shows that the width of the strip is $O(r)$.

5.2. Choice of origin. Choose $x_{0} \in \Gamma \backslash G$. Let $\mathcal{U}_{x_{0}}^{\prime} \subset K / M$ denote the set of $k \in K$ such that $\pi\left(x_{0} k\right) \in E$ where $E$ is as in Lemma 5.2. Note that for almost all choices of $x_{0}$,

$$
m_{K}\left(\mathcal{U}_{x_{0}}^{\prime}\right)=1
$$

(where $m_{K}$ is the $K$-invariant measure on $K / M$ ). We choose $x_{0}$ so that (33) holds, and denote $\mathcal{U}_{x_{0}}^{\prime}$ by $\mathcal{U}^{\prime}$. For simplicity of notation we set $x_{0}=e$ (this can always be accomplished by replacing $\Gamma$ with a suitable conjugate).

Let $\mathcal{U}_{1}$ denote the set of points $k$ in $K / M$ such that $k \in \mathcal{U}^{\prime}$ and for all $\sigma \subset \Delta$, $m_{K}\left(\mathcal{U}^{\prime} \cap k K_{\sigma}\right)=m_{K}\left(k K_{\sigma}\right)$. By Fubini's theorem, $m_{K}\left(\mathcal{U}_{1}\right)=1$.

We normalize $\phi$ so that $\phi(e)=e$.

5.3. Angles between flats. The following lemma is the main estimate used in the proof of Step 5 .

Lemma 5.3 (Angle between flats is bi-Hölder). Suppose $\alpha \in \Delta, k \in \mathcal{U}_{1}$, and $x_{1}, x_{2}$ $\in k K_{\alpha} \cap \mathcal{U}^{\prime}$. Then,

$$
C^{-1} d_{K}\left(x_{1}, x_{2}\right)^{1 / \lambda} \leq d_{K}\left(\phi_{0}\left(x_{1}\right), \phi_{0}\left(x_{2}\right)\right) \leq C d_{K}\left(x_{1}, x_{2}\right)^{\lambda}
$$

where $C=C(k)$ and $\lambda$ is independent of $x_{1}, x_{2}$ and $k$.

The proof uses the following:

Lemma 5.4. Suppose $F_{1}, F_{2} \in \mathcal{F}^{*}$ are flats, and $\mathfrak{C}_{1} \subset F_{1}$ and $\mathfrak{C}_{2} \subset F_{2}$ are Weyl chambers both based at the point $p \in F_{1} \cap F_{2}$. Suppose $\mathfrak{C}_{1}$ and $\mathfrak{C}_{2}$ share a codimension 1 wall, and $p \in \Omega_{F_{1}}^{*} \cap \Omega_{F_{2}}^{*}$. Suppose $p^{\prime} \in B(\phi(p), O(N))$. Let $g_{0} \in G$ be such that $g_{0} \cdot p=e$, and let $g_{0}^{\prime} \in G$ be such that $g_{0}^{\prime} \cdot p^{\prime}=e$. Then,

$$
C^{-1} d_{K}\left(g_{0} \mathfrak{C}_{1}, g_{0} \mathfrak{C}_{2}\right)^{1 / \lambda} \leq d_{K}\left(g_{0}^{\prime} \cdot \phi_{0}\left(\mathfrak{C}_{1}\right), g_{0}^{\prime} \cdot \phi_{0}\left(\mathfrak{C}_{2}\right)\right) \leq C d_{K}\left(g_{0} \mathfrak{C}_{1}, g_{0} \mathfrak{C}_{2}\right)^{\lambda}
$$

where $C$ and $\lambda$ are independent of $\mathfrak{C}_{1}, \mathfrak{C}_{2}, F_{1}, F_{2}, g_{0}, g_{0}^{\prime}$ and $p$.

Remarks. The assumption that $\mathfrak{C}_{1}$ and $\mathfrak{C}_{2}$ share a codimension 1 wall is not necessary. Also, if $p=e$, then we can let $g_{0}=1, g_{0}^{\prime}=1$, and the estimate of Step 5 follows immediately from (34). However, in general, we may not assume that for almost all $k \in \mathcal{U}_{1}, e \in \Omega_{\pi(k A)}^{*}$, so Lemma 5.4 cannot be applied directly.

Proof of Lemma 5.4. By Proposition 3.4, part (b), there exist Weyl chambers $\mathfrak{C}_{1}^{\prime \prime}$, $\mathfrak{C}_{2}^{\prime \prime}$ such that $\tilde{\phi}\left(\mathfrak{C}_{i}\right) \sim_{\lambda \epsilon, \phi(x), \rho} \mathfrak{C}_{i}^{\prime \prime}$. By Step 3, since $p \in \Omega_{F_{i}}, d\left(p^{\prime}, F_{i}^{\prime}\right)=O(N)$. By Lemma B.8 for $i=1,2$ we may replace $\mathfrak{C}_{i}^{\prime \prime}$ by an equivalent chamber $\mathfrak{C}_{i}^{\prime \prime}$ such that $\mathfrak{C}_{1}^{\prime}$ and $\mathfrak{C}_{2}^{\prime}$ are both based at $p^{\prime}$, and still $\tilde{\phi}\left(\mathfrak{C}_{i}\right) \sim_{\lambda \epsilon, p^{\prime}, \rho^{\prime}} \mathfrak{C}_{i}^{\prime}$ for any $\rho^{\prime} \gg \max (\rho, N)$. For $i=1,2$, let $\mathfrak{D}_{i}=g_{0} \mathfrak{C}_{i}, \mathfrak{D}_{i}^{\prime}=g_{0}^{\prime} \mathfrak{C}_{i}^{\prime}$. Then all four of these Weyl chambers are based at $e$, hence there exist $k_{i}, k_{i}^{\prime} \in K$ such that $\mathfrak{D}_{i}=\pi\left(k_{i} A^{+}\right), \mathfrak{D}_{i}^{\prime}=\pi\left(k_{i}^{\prime} A^{+}\right), i=1,2$. By Step $4, \mathfrak{C}_{1}^{\prime}$ and $\mathfrak{C}_{2}^{\prime}$ (hence also $\mathfrak{D}_{1}^{\prime}$ and $\mathfrak{D}_{2}^{\prime}$ ) share a codimension 1 wall. Let $\theta=d_{K}\left(k_{1}, k_{2}\right), \theta^{\prime}=d_{K}\left(k_{1}^{\prime}, k_{2}^{\prime}\right)$. Hence (34) is equivalent to $C^{-1} \theta^{1 / \lambda} \leq \theta^{\prime} \leq C \theta^{\lambda}$.

Let $\tilde{\psi}=g_{0}^{\prime} \circ \tilde{\phi} \circ g_{0}^{-1}$. Then, $\tilde{\psi}: \mathfrak{D}_{1} \cup \mathfrak{D}_{2} \rightarrow \operatorname{Nbhd}\left(\mathfrak{D}_{1}^{\prime}, N\right) \cup \operatorname{Nbhd}\left(\mathfrak{D}_{2}^{\prime}, N\right)$ is a graded quasi-isometric embedding, and $\tilde{\psi}\left(\mathfrak{D}_{i}\right) \sim_{\lambda \epsilon, e, \rho^{\prime}} \mathfrak{D}_{i}^{\prime}, i=1,2$. It follows from 
Proposition 3.4 (cf. the proof of Lemma 4.3) that

$$
\tilde{\psi}\left(\partial \mathfrak{D}_{i}\right) \sim_{\lambda \epsilon, e, \rho^{\prime}} \partial \mathfrak{D}_{i}^{\prime} .
$$

Let $Q \gg 1$ and $0<\nu_{3}<1$ be as in Lemma B.6. In the following paragraph, the implicit constants do not depend on $\nu_{3}$ and $Q$. Without loss of generality, $\theta$ is sufficiently small so that $\nu_{3}|\log \theta| \gg \max \left(Q / \epsilon, N / \epsilon, \rho^{\prime} / \epsilon\right)$. By Lemma B.6, there exist points $z_{i} \in \mathfrak{D}_{i}$ such that $d\left(z_{1}, z_{2}\right) \leq Q, d\left(z_{i}, e\right)=O(|\log \theta|)$ and $d\left(z_{i}, \partial \mathfrak{D}_{i}\right) \geq$ $\nu_{3}|\log \theta|$. Then, since $\tilde{\psi}$ is a graded q.i. embedding and by $(35), d\left(\tilde{\psi}\left(z_{i}\right), \partial \mathfrak{D}_{i}^{\prime}\right) \succ$ $\nu_{3}|\log \theta|$. Also,

$$
d\left(\tilde{\psi}\left(z_{1}\right), \tilde{\psi}\left(z_{2}\right)\right)=O\left(d\left(z_{1}, z_{2}\right)\right)+O\left(\epsilon d\left(z_{1}, e\right)\right)+O\left(\epsilon d\left(z_{2}, e\right)\right) \ll \nu_{3}|\log \theta| .
$$

Let $z_{i}^{\prime}$ denote the nearest point projection of $\tilde{\psi}\left(z_{i}\right)$ onto $\mathfrak{D}_{i}^{\prime}$. Then, there exists $r \succ$ $\nu_{3}|\log \theta|$ (with the implied constant depending only on $\kappa, X$ ) such that $d\left(z_{1}^{\prime}, z_{2}^{\prime}\right) \leq$ $\lambda_{2} r, d\left(z_{i}^{\prime}, \partial \mathfrak{D}_{i}^{\prime}\right) \geq \nu^{\prime} r$, where $\lambda_{2}, \nu^{\prime}$ are as in Lemma B.4. Hence, by Lemma B.4, $\theta^{\prime} \leq c e^{-\nu r}$. Hence, $\theta^{\prime} \leq c \theta^{\lambda}$, where $\lambda$ depends only on $\kappa, X$ (and $\nu_{3}$, but $\nu_{3}$ and $Q$ depend only on $X$ ).

The opposite inequality follows from applying the same argument to the map $\tilde{\psi}^{*}: \mathfrak{D}_{1}^{\prime} \cup \mathfrak{D}_{2}^{\prime} \rightarrow \mathfrak{D}_{1} \cup \mathfrak{D}_{2}$ which is the coarse inverse of $\tilde{\psi}$ composed with nearest point projection.

Proof of Lemma 5.3. We write $x_{1}=k k_{1}, x_{2}=k k_{2}$, where $k_{i} \in K_{\alpha}$. Let $L=$ $\pi\left(k A_{\alpha}\right)$. Since $k \in \mathcal{U}_{1}, L \in \mathcal{L}$, it follows from Lemma 5.2 that there exists a hyperplane $L^{\prime}$ such that $\phi\left(\Omega_{L}\right) \subset \operatorname{Nbhd}\left(L^{\prime}, N\right)$. Pick a point $x_{0}$ in $\Omega_{L}$. Then, in particular, $\phi\left(x_{0}\right) \in \operatorname{Nbhd}\left(L^{\prime}, N\right)$. Let $x_{0}^{\prime} \in G$ be such that $L^{\prime}=\pi\left(x_{0}^{\prime} A_{\beta}\right)$ for some $\beta \in \Sigma$, and $\pi\left(x_{0}^{\prime}\right) \in L$ is the closest point to $\phi\left(x_{0}\right)$. Let $g_{1}^{\prime}=x_{0}^{\prime-1}$, so that $g_{1}^{\prime} \pi\left(x_{0}^{\prime}\right)=e$. Note that $g_{1}^{\prime}, x_{0}^{\prime}$ and $x_{0}$ are independent of $x_{1}$ and $x_{2}$.

Since $k k_{i} \in \mathcal{U}^{\prime}$, by Lemma 5.2 , parts (d) and (e),

$$
\liminf _{r \rightarrow \infty}\left|\Omega_{F_{1}} \cap \Omega_{F_{2}} \cap \Omega_{L} \cap B\left(x_{0}, r\right)\right| \geq(1-3|\Sigma| \epsilon)\left|B\left(x_{0}, r\right) \cap L\right| .
$$

Hence there exists a point $p \in L \cap \Omega_{F_{1}} \cap \Omega_{F_{2}} \cap \Omega_{L}$.

Since $p \in L, p=\pi(k a)$ for some $a \in A_{\alpha}$. For $i=1,2$ let $\mathfrak{C}_{i}$ denote the Weyl chambers $\pi\left(k a k_{i} A^{+}\right)$. Then the $\mathfrak{C}_{i}$ are based at $p$. Since $k_{i} \in K_{\alpha}, a \in A_{\alpha}$ and $K_{\alpha}$ and $A_{\alpha}$ commute, $\mathfrak{C}_{i}$ is equivalent to $\pi\left(k k_{i} A^{+}\right)$. Hence $d_{K}\left(\mathfrak{C}_{1}, \mathfrak{C}_{2}\right)=d_{K}\left(k_{1}, k_{2}\right)$, and

$$
\phi_{0}\left(x_{i}\right)=\phi_{0}\left(\pi\left(x_{i} A^{+}\right)\right)=\phi_{0}\left(\mathfrak{C}_{i}\right) .
$$

Let $g_{0}=(k a)^{-1}$, so that $g_{0} p=e$. Then $g_{0} \mathfrak{C}_{i}=\pi\left(k_{i} A^{+}\right)$, hence

$$
d_{K}\left(g_{0} \mathfrak{C}_{1}, g_{0} \mathfrak{C}_{2}\right)=d_{K}\left(k_{1}, k_{2}\right)=d_{K}\left(x_{1}, x_{2}\right) .
$$

Now let $p^{\prime}$ be the closest point in $L^{\prime}$ to $\phi(p)$. We have $d\left(p^{\prime}, \phi(p)\right) \leq N$. Also, since $p^{\prime} \in L^{\prime}$, there exists $a^{\prime} \in A_{\beta}$ such that $p^{\prime}=\pi\left(x_{0}^{\prime} a^{\prime}\right)$. Let $g_{0}^{\prime}=\left(x_{0}^{\prime} a^{\prime}\right)^{-1}$ so that $g_{0}^{\prime} p^{\prime}=e$.

For $i=1,2$, let $\mathfrak{C}_{i}^{\prime}$ denote the Weyl chamber in the equivalence class $\phi_{0}\left(\mathfrak{C}_{i}\right)$ based at $p^{\prime}$. Let $L_{+}$denote the common codimension 1 wall of $\mathfrak{C}_{1}$ and $\mathfrak{C}_{2}$. By Step $4, \mathfrak{C}_{1}^{\prime}$ and $\mathfrak{C}_{2}^{\prime}$ share a common codimension 1 wall $H_{+}$, and by Lemma 4.3 , $\tilde{\phi}\left(L_{+}\right) \sim_{\lambda \epsilon} H_{+}$. But $\tilde{\phi}\left(L_{+}\right) \subset \tilde{\phi}(L) \sim_{\lambda \epsilon} L^{\prime}$, hence outside a large ball, $H_{+} \subset L^{\prime}[\lambda \epsilon]$. Since $H_{+}$is based at $p^{\prime}$ and $p^{\prime} \in L^{\prime}$, this implies that $H_{+} \subset L^{\prime}$. Hence $\mathfrak{C}_{1}^{\prime}$ and $\mathfrak{C}_{2}^{\prime}$ share a codimension 1 wall contained in $L^{\prime}$.

Hence $\mathfrak{C}_{i}^{\prime}=\pi\left(x_{0}^{\prime} a^{\prime} k_{i}^{\prime} A^{+}\right)$, where $k_{i}^{\prime} \in K_{\beta}$. Let $\mathfrak{D}_{i}^{\prime}=\pi\left(x_{0}^{\prime} k_{i}^{\prime} A^{+}\right)$, so that $\mathfrak{D}_{i}^{\prime}$ is based at $\pi\left(x_{0}^{\prime}\right)$. Since $a^{\prime} \in A_{\beta}, k_{i}^{\prime} \in K_{\beta}$ and $A_{\beta}$ and $K_{\beta}$ commute, $\mathfrak{C}_{i}^{\prime}$ is equivalent 
to $\mathfrak{D}_{i}^{\prime}$, so we may write $\phi_{0}\left(\mathfrak{C}_{i}\right)=\mathfrak{D}_{i}^{\prime}$. Also note that $g_{0}^{\prime} \mathfrak{C}_{i}^{\prime}=\pi\left(k_{i}^{\prime} A^{+}\right)=g_{1}^{\prime} \mathfrak{D}_{i}^{\prime}$, so we may write

$$
g_{0}^{\prime} \cdot \phi_{0}\left(\mathfrak{C}_{i}\right)=g_{0}^{\prime} \mathfrak{C}_{i}^{\prime}=g_{1}^{\prime} \mathfrak{D}_{i}^{\prime}=g_{1}^{\prime} \cdot \phi_{0}\left(\mathfrak{C}_{i}\right)
$$

By Lemma 5.4,

$$
C^{-1} d_{K}\left(g_{0} \mathfrak{C}_{1}, g_{0} \mathfrak{C}_{2}\right)^{1 / \lambda} \leq d_{K}\left(g_{0}^{\prime} \cdot \phi_{0}\left(\mathfrak{C}_{1}\right), g_{0}^{\prime} \cdot \phi_{0}\left(\mathfrak{C}_{2}\right)\right) \leq C d_{K}\left(g_{0} \mathfrak{C}_{1}, g_{0} \mathfrak{C}_{2}\right)^{\lambda} .
$$

Combining (36), (37), (38) and (39), we get

$$
C^{-1} d_{K}\left(x_{1}, x_{2}\right)^{1 / \lambda} \leq d_{K}\left(g_{1}^{\prime} \cdot \phi_{0}\left(x_{1}\right), g_{1}^{\prime} \cdot \phi_{0}\left(x_{2}\right)\right) \leq C d_{K}\left(x_{1}, x_{2}\right)^{\lambda} .
$$

Since $g_{1}^{\prime}$ acts as a diffeomorphism of the boundary, and is independent of $x_{1}, x_{2}$, this implies

$$
c_{2}^{\prime \prime} d_{K}\left(x_{1}, x_{2}\right)^{1 / \lambda} \leq d_{K}\left(\phi_{0}\left(x_{1}\right), \phi_{0}\left(x_{2}\right)\right) \leq c_{1}^{\prime \prime} d_{K}\left(x_{1}, x_{2}\right)^{\lambda}
$$

where $c_{1}^{\prime \prime}, c_{2}^{\prime \prime}$ depend on $g_{1}^{\prime}$, hence $L$.

5.4. Differentiability and $\mathcal{T}(X)$. In this section we complete the proof of Step 5 . In view of Lemma 5.3 and Step 4 it is enough to prove the following:

Proposition 5.5. Let $\mathcal{U}_{1} \subset \hat{X}$ be a subset with $m_{K}\left(\mathcal{U}_{1}\right)=1$, and let $\phi_{0}: \mathcal{U}_{1} \rightarrow \hat{X}$ be a Tits map defined on $\mathcal{U}_{1}$. Suppose $\phi_{0}$ satisfies the conclusion of Lemma 5.3. Then, there exists a subset $\mathcal{U}_{2} \subset \mathcal{U}_{1}$ with $m_{K}\left(\mathcal{U}_{2}\right)=1$ such that the restriction of $\phi_{0}$ to $\mathcal{U}_{2}$ is bi-Holder.

The proof of Proposition 5.5 does not involve either quasi-isometries or ergodic theory; instead it deals with some extra structure of the building $\mathcal{T}(X)$. Lemma 5.3 does not immediately imply the statement of Step 5 because of the restriction to Weyl chambers with a common codimension 1 wall $L$, and because the constant $C$ depends on $L$. The idea of the proof is to use Step 4 to show that finitely many applications of Lemma 5.3 imply the statement for $\phi_{0}$.

Lemma 5.6 (Image of uniformly distributed set under $\phi_{0}$ is dense). Let $E$ be a subset of full measure in $K / M$. Then, $\phi_{0}\left(E \cap \mathcal{U}_{1}\right)$ is dense in $K / M$.

Proof. Without loss of generality, we may assume that $E \subset \mathcal{U}_{1}$. Let $E_{1}=E \cap\{k \in$ $\left.K / M: m_{K_{\alpha}}\left(k K_{\alpha} \cap E\right)=1, \forall \alpha \in \Delta\right\}$, where $m_{K_{\alpha}}$ is normalized Haar measure on $K_{\alpha} / M$, and we denote the push forward measure on $k K_{\alpha} / M$ also by $m_{K_{\alpha}}$. Then, by Fubini's theorem, $m_{K}\left(E_{1}\right)=1$.

Suppose $k \in E_{1}$. Then we claim that $\phi_{0}(E)$ is dense in $\bigcup_{\alpha \in \Delta} k^{\prime} K_{\alpha}$, where $k^{\prime}=\phi_{0}(k)$. Indeed by Step 4 for each $\alpha \in \Delta$ there exists $\beta \in \Delta$ such that $\phi_{0}\left(k K_{\alpha} \cap E\right) \subset k^{\prime} K_{\beta}$, and by Lemma 5.3 the associated map $\psi_{\alpha}=\ell_{k^{\prime}}^{-1} \circ \phi_{0} \circ$ $\ell_{k}: K_{\alpha} / M \rightarrow K_{\beta} / M$ (where $\ell_{k}$ denotes left translation by $k$ ) is bi-Hölder on its domain of definition (i.e. $k^{-1} \mathcal{U}_{1} \cap K_{\alpha} / M$ ). Hence $\psi_{\alpha}$ extends to an injective continuous map $\overline{\psi_{\alpha}}: K_{\alpha} / M \rightarrow K_{\beta} / M$. Choose $\alpha$ so that $K_{\alpha} / M$ has maximal dimension; then by invariance of domain (see e.g. [Mu, Theorem 36.5]) $\overline{\psi_{\alpha}}$ is a homeomorphism, and $\operatorname{dim}\left(K_{\beta} / M\right)=\operatorname{dim}\left(K_{\alpha} / M\right)$. Since $\overline{\psi_{\alpha}}$ is a homeomorphism, the image of the full measure (hence dense) set $E \cap k K_{\alpha} / M$ is dense. Proceeding in this way from maximal to minimal dimension of $K_{\alpha} / M$, we see that for any $\alpha \in \Delta$, $\phi_{0}\left(E \cap k K_{\alpha} / M\right)$ is dense in $\phi_{0}(k) K_{\beta} / M$. This implies the claim.

Similarly, for $j \geq 2$, let $E_{j}=E_{j-1} \cap\left\{k \in K / M \quad: m_{K_{\alpha}}\left(k K_{\alpha} \cap E_{j-1}\right)=1\right\}$. Then for any $j, m_{K}\left(E_{j}\right)=1$, and for any $k \in E_{j}, \phi_{0}\left(E_{j-1} \cap k \cup_{\alpha \in \Delta} K_{\alpha} / M\right)$ is dense in $\bigcup_{\alpha \in \Delta} \phi_{0}(k) K_{\alpha}$. 
To finish the proof of the lemma, note that the maximal combinatorial distance in the Tits building is $\ell<\infty$. Hence for every two elements $x, y \in K / M$ there exists a gallery of length $\ell$ connecting $x$ and $y$, i.e. a sequence of elements $x=$ $x_{0}, x_{1}, \ldots, x_{\ell}=y$, with $x_{i+1} \in x_{i} K_{\alpha_{i}}$, with $\alpha_{i} \in \Delta$. We fix $x \in \phi_{0}\left(E_{\ell}\right)$, and let $y$ denote an arbitrary point in $K / M$. By the claim, there exists $x_{1}^{\prime} \in \phi_{0}\left(E_{\ell-1}\right)$ arbitrarily close to $x_{1}$. By the same argument, there exists $x_{2}^{\prime} \in \phi_{0}\left(E_{\ell-2}\right)$ arbitrarily close to $x_{2}$, etc. Thus there exists a point $x_{\ell}^{\prime} \in \phi_{0}(E)$ arbitrarily close to $y$. This proves the lemma.

Lemma 5.7 (The function $f_{\alpha}$ ). Suppose $t \in K_{\alpha}, s \in \mathcal{S}$, and $y=t s \notin \mathcal{S}$.

(a) $t M$ is uniquely determined by $y$; we denote it by $f_{\alpha}(y)$. Also for $m \in M$, $f_{\alpha}(y m)=f_{\alpha}(y)$. There is a well-defined function $f_{\alpha}: \mathcal{S}^{c} / M \rightarrow K_{\alpha} / M$.

(b) There exists a Zariski closed proper subset $S_{\alpha}$ of $K / M$ such that the restriction of $f_{\alpha}$ to $\mathcal{S}_{\alpha}^{c}$ is real analytic.

(c) $f_{\alpha}(m y)=m f_{\alpha}(y)$ for $m \in M$ and $y \in \mathcal{S}^{c}$.

Proof. Since $s \in \mathcal{S}, s \in K \cap B w^{\prime} B$ for some $w^{\prime} \in W$, where $w^{\prime}=w_{\alpha_{1}} \cdots w_{\alpha_{r}}$ and $r<\ell$, the maximal combinatorial distance (see the "algebraic preliminaries"). Then $s=k_{\alpha_{1}} \cdots k_{\alpha_{r}}$ where $k_{\alpha_{i}} \in K \cap B w_{\alpha_{i}} B=K_{\alpha_{i}}$. Consider the path (of length $r+1) x_{0}, \ldots, x_{r+1}$ where $x_{0}=e M, x_{1}=t M, x_{i}=t k_{1} \cdots k_{i-1} M, 1 \leq i \leq r+1$, so that $x_{r+1}=y M$. Thus $r+1 \geq d_{c}(y M, e M)$. But since $y \notin \mathcal{S}, d_{c}(y, e)=\ell$, hence $r=\ell-1$. Hence $\left(x_{0}, \ldots, x_{r+1}\right)$ is a minimal gallery connecting the chambers $e M$ and $y M$.

Since apartments in the Tits building are convex (see [B, Proposition, $\S I V .2$, p. 88]), any minimal gallery connecting two chambers always lies in an apartment containing the two chambers. The apartment $\mathcal{A}$ containing $e M$ and $y M$ is unique since $y \notin \mathcal{S}$. Thus the chamber $x_{1}=t M$ is uniquely determined as the chamber in $\mathcal{A}$ which is adjacent to the chamber $e M$ and obtained from it by reflection in the wall associated to $w_{\alpha}$. Hence $t M \in K_{\alpha} / M$ is determined uniquely by $y M \in \mathcal{S}^{c} / M$. This proves (a).

Since $r=\ell-1, s$ lies in the interior of a maximal nonopen Bruhat cell. Let $K_{\alpha}^{\prime} \subset K_{\alpha}$ be a submanifold containing $t$ transversal to $t M$, so that near $t M, K_{\alpha} / M$ is locally diffeomorphic to $K_{\alpha}^{\prime}$. Then the product map $\Psi_{\alpha}: K_{\alpha}^{\prime} \times \mathcal{S} / M \rightarrow K / M$ is real analytic; by part (a) it is one-to-one near $(t, s)$, and $f_{\alpha}\left(\Psi_{\alpha}\left(t^{\prime}, s^{\prime} M\right)\right)=t^{\prime} M$. Since $\Psi_{\alpha}$ is one-to-one near $(t, s)$, we can have $D \Psi_{\alpha}=0$ only on a (possibly empty) proper Zariski closed subset $V_{\alpha}$; then by the implicit function theorem, $f_{\alpha}$ is differentiable away from the proper Zariski closed subset $\Psi_{\alpha}\left(V_{\alpha}\right)$. It is easy to check that $\Psi_{\alpha}\left(V_{\alpha}\right)$ does not in fact depend on the choice of $K_{\alpha}^{\prime}$. This proves (b).

To show (c) note that if $m \in M$ and $t=f_{\alpha}(y)$, i.e. $t \in K_{\alpha} / M$ and (eM, $t M$, $\left.t k_{1} M, \ldots, t k_{1} \cdots k_{\ell-1} M=y M\right)$ is a minimal gallery connecting $e M$ and $y M$, then ( $\left.m e M, m t M, m t k_{1} M, \ldots, m t k_{1} \cdots k_{\ell-1} M=m y M\right)$ is a minimal gallery connecting $m e M=e M$ to $m y M$. Hence $f_{\alpha}(m y)=m f_{\alpha}(y)$ for $m \in M$.

For $k \in K, \alpha \in \Delta$, we write $f_{k, \alpha}(y)=f_{\alpha}\left(k^{-1} y\right)$.

Lemma 5.8 (Coordinates on $K / M)$. (i) For any $x, y \in K / M$ with $x \neq y$, there exists $k \in K$ and $\alpha \in \Delta$ such that $f_{k, \alpha}(x) \neq f_{k, \alpha}(y)$.

(ii) For any $x \in K / M$ there exist $\alpha_{1}, \ldots, \alpha_{m} \in \Delta$ and an open full measure subset $V$ of $\prod_{i=1}^{m} K$, invariant under right-multiplication by $\prod_{i=1}^{m} M$, such that for any $\left(k_{1}, \ldots, k_{m}\right) \in V$ there exists an open neighborhood $U$ of $x$ 
such that the map $\Phi: U \rightarrow K_{\alpha_{1}} / M \times \cdots \times K_{\alpha_{m}} / M$ given by $\Phi(y)=$ $\left(f_{k_{1}, \alpha_{1}}(y), \ldots, f_{k_{m}, \alpha_{m}}(y)\right)$ is a diffeomorphism onto its image.

Proof. To prove (i), we may assume without loss of generality that $x=e$. Suppose $f_{\alpha}\left(k^{-1}\right)=f_{\alpha}\left(k^{-1} y\right)$ for all $\alpha$ and all $k$ such that both sides are defined. Now suppose $s \in \mathcal{S}_{\ell-1}$ where $\mathcal{S}_{\ell-1}$ is the subset of $\mathcal{S}$ with $d_{c}(s, e)=\ell-1$. Then there exists $\alpha \in \Delta$ such that $s$ forms a minimal gallery together with almost all elements $t \in K_{\alpha}$; then $f_{\alpha}(t s)$ is defined. Writing $k^{-1}=t s$, we get $t=f_{\alpha}\left(k^{-1}\right)=f_{\alpha}\left(k^{-1} y\right)=$ $f_{\alpha}(t s y)$, i.e. $s y \in \mathcal{S}$. Note that $f_{\alpha}(t s y)$ is defined for almost all $t$ if $s$ is in a dense subset $\mathcal{S}_{\ell-1}^{\prime}$ of $\mathcal{S}_{\ell-1}$. Hence we have $s y \in \mathcal{S}$ for all $s \in \mathcal{S}_{\ell-1}^{\prime}$. However, $\mathcal{S}$ is closed and $\mathcal{S}_{\ell-1}^{\prime}$ is dense in $\mathcal{S}$. Hence, $\mathcal{S} y \subset \mathcal{S}$. But this is clearly impossible unless $y=e$. Indeed, let $\mathcal{A}$ be an apartment containing $e$ and $y^{-1}$, and let $w$ denote the chamber in $\mathcal{A}$ opposite $e$. Then there exists a minimal gallery connecting $e$ and $w$ and passing though $y^{-1}$ (see [B, Lemma, §IV.5, p. 92]). Thus $w=y^{-1} s$ for some $s \in \mathcal{S}$, hence $w^{-1}=s^{-1} y$. Since $\mathcal{S}^{-1}=\mathcal{S}, s^{-1} \in \mathcal{S}$. However $w^{-1} \notin \mathcal{S}$, hence we have a contradiction. This proves (i). Note that (i) implies that for any $x \neq y$ there exists $\alpha \in \Delta$ such that the set $\left\{k \in K: f_{k, \alpha}(x) \neq f_{k, \alpha}(y)\right\}$ is open and dense $K$.

Suppose (ii) does not hold. Then there exists a point $y \in K / M$ and a vector $X \in T_{y}(K / M)$ such that for all $\alpha \in \Delta$ and all $k$ with $k^{-1} y \in \mathcal{S}_{\alpha}^{c}, D f_{k, \alpha}(X)=0$. Since for any $k_{0} \in K, f_{k_{0} k, \alpha}\left(k_{0} y\right)=f_{k, \alpha}(y)$, the vector $k_{0} X \in T_{k_{0} y}(K / M)$ satisfies the same condition, i.e. $D f_{k, \alpha}\left(k_{0} X\right)=0$ for all $\alpha \in \Delta$ and all $k \in K$ with $k^{-1}\left(k_{0} y\right) \in \mathcal{S}_{\alpha}^{c}$. We thus define a vector field on $K / M$ by choosing the vector $k_{0} X$ in $T_{k_{0} y}(K / M)$. Let $\gamma:[0,1] \rightarrow K / M$ be a flow line for this vector field; then $f_{k, \alpha}(\gamma(0))=f_{k, \alpha}(\gamma(1))$ for all $\alpha \in \Delta$ and $k \in K$ such that both sides are defined. This contradicts (i).

Proof of Proposition 5.5. For $k \in \mathcal{U}_{1}, \phi_{0}\left(k K_{\alpha}\right) \subset \phi_{0}(k) K_{\beta}$, for some $\beta \in \Delta$.

Suppose $x \in \mathcal{U}_{1} \cap \mathcal{S}^{c}$. Let $t=f_{k, \alpha}(x)$, i.e. $k^{-1} x=t s, s \in \mathcal{S}$. Write $s=k_{1} \cdots k_{\ell-1}$ where $k_{i} \in K_{\alpha_{i}}$ for some $\alpha_{i} \in \Delta$. Then the gallery

$$
\left(e M, t M, t k_{1} M, t k_{1} k_{2} M, \ldots, t k_{1} \cdots k_{\ell-1} M\right)
$$

is a minimal gallery connecting $e$ and $k^{-1} x$. Hence

$$
\left(k M, k t M, k t k_{1} M, k t k_{1} k_{2} M, \ldots, k t k_{1} \cdots k_{\ell-1} M\right)
$$

is a minimal gallery connecting $k$ and $x$.

Suppose this gallery lies completely in $\mathcal{U}_{1}$ (this will happen for $x \in H_{k, \alpha}$ where $H_{k, \alpha} \subset K / M$ has full measure). Then

$$
\left(\phi_{0}(k) M, \phi_{0}(k t) M, \phi_{0}\left(k t k_{1}\right) M, \phi_{0}\left(k t k_{1} k_{2}\right) M, \ldots, \phi_{0}\left(k t k_{1} \cdots k_{\ell-1}\right) M\right)
$$

is also a gallery connecting $\phi_{0}(k)$ and $\phi_{0}(x)$. If $\phi_{0}(x) \notin \phi_{0}(k) \mathcal{S}$, then this gallery is minimal, and (since $\left.\phi_{0}(k t) \subset \phi_{0}(k) K_{\beta}\right), f_{\phi_{0}(k), \beta}\left(\phi_{0}(x)\right)=\phi_{0}(k)^{-1} \phi_{0}(k t)$. Hence

$$
f_{\phi_{0}(k), \beta}\left(\phi_{0}(x)\right)=\phi_{0}(k)^{-1} \phi_{0}\left(k f_{k, \alpha}(x)\right), \quad \text { for } x \in H_{k, \alpha} \text {. }
$$

Choose $x \in \mathcal{U}_{1}$. By Lemma 5.8 for $1 \leq i \leq m$ there exist subsets $E_{i} \subset K$ of full measure such that if $k_{i} \in E_{i}$ for $1 \leq i \leq m$, then condition (ii) of Lemma 5.8 holds for $\left(k_{1}, \ldots, k_{m}\right)$ and a neighborhood $U_{x}$ of $x$. Now let $V$ be as in Lemma 5.8, part (ii), but for the point $\phi_{0}(x)$ instead of $x$. By Lemma 5.6 the set $\prod_{i=1}^{m} \phi_{0}\left(E_{i}\right)$ is dense in $\prod_{i=1}^{m} K / M$. Since $V$ is open there exist $k_{i} \in E_{i}$ such that $\left(\phi_{0}\left(k_{1}\right), \ldots, \phi_{0}\left(k_{m}\right)\right) \in$ $V$. Thus we may assume that the condition of part (ii) of Lemma 5.8 holds for $x$, the $k_{i}$, and for some neighborhood of $U_{x}$ of $x$, and also for $\phi_{0}(x)$, the $\phi_{0}\left(k_{i}\right)$, and 
some neighborhood $U_{\phi_{0}(x)}$ of $\phi_{0}(x)$. Then for $y \in U_{x} \cap \phi_{0}^{-1}\left(U_{\phi_{0}(x)}\right) \cap_{i=1}^{m} H_{k_{i}, \alpha_{i}}$, using (41)

$$
\phi_{0}(y)=\Phi^{-1}\left(\phi_{0}\left(k_{1}\right)^{-1} \phi_{0}\left(k_{1} f_{k_{1}, \alpha_{1}}(y)\right), \ldots, \phi_{0}\left(k_{m}\right)^{-1} \phi_{0}\left(k_{m} f_{k_{m}, \alpha_{m}}(y)\right)\right)
$$

where $\Phi$ is the diffeomorphism from Lemma 5.8 at the point $\phi_{0}(x)$. By Lemma 5.3, the restriction of $\phi_{0}$ to $k_{i} K_{\alpha_{i}} \cap \mathcal{U}_{1}$ is bi-Hölder. Then by (42), $\phi_{0}$, restricted to $U_{x} \cap \phi_{0}^{-1}\left(U_{\phi_{0}(x)}\right) \cap_{i=1}^{m} H_{k_{i}, \alpha_{i}}$ is bi-Hölder, hence uniformly continuous. Then, by compactness, there exists a full measure subset $\mathcal{U}_{2} \subset \mathcal{U}_{1}$ such that $\phi_{0}$ restricted to $\mathcal{U}_{2}$ is uniformly continuous. Hence $\phi_{0} \mid \mathcal{U}_{2}$ extends to a continuous map $\overline{\phi_{0}}$ defined on all of $K / M$. It is clear that $\overline{\phi_{0}}$ is locally injective; it remains to show that $\overline{\phi_{0}}$ is injective.

For every $x, y \in K$, there exist $k, \alpha$ so that (i) of Lemma 5.8 holds; for fixed $k$ and $\alpha$ the set of $(x, y)$ which satisfies this condition is open. Hence, using the compactness of $K \times K$ there exists a finite collection of $k_{i} \in \mathcal{U}_{1}$ such that if $f_{k_{i}, \alpha_{i}}(x)=f_{k_{i}, \alpha_{i}}(y)$ for all $i$ for which these functions are defined, then $x=y$.

Suppose $\overline{\phi_{0}}$ is not injective. Then, since $\overline{\phi_{0}}$ is locally injective, there exist disjoint neighborhoods $U$ and $V$ such that $\overline{\phi_{0}}(U)=\overline{\phi_{0}}(V)$. Then, after passing to smaller neighborhoods if needed, there exists $i$ such that $f_{k_{i}, \alpha_{i}}(U)$ and $f_{k_{i}, \alpha_{i}}(V)$ are disjoint. Since $\phi_{0}$ restricted to $k_{i} K_{\alpha_{i}} \cap \mathcal{U}_{1}$ is bi-Hölder, $\overline{\phi_{0}}$ restricted to $k_{i} K_{\alpha_{i}}$ is injective. Hence $\overline{\phi_{0}}\left(k_{i} f_{k_{i}, \alpha_{i}}(U)\right)$ and $\overline{\phi_{0}}\left(k_{i} f_{k_{i}, \alpha_{i}}(V)\right)$ are disjoint.

The fact that $\overline{\phi_{0}}$ is locally onto implies that there exist $x \in U, y \in V$ such that $z=\overline{\phi_{0}}(x)=\overline{\phi_{0}}(y)$. By perturbing $k_{i}$ we may make sure that $f_{\phi_{0}\left(k_{i}\right), \beta}(z)$ makes sense for all $\beta \in \Delta$, and still $\overline{\phi_{0}}\left(k_{i} f_{k_{i}, \alpha_{i}}(x)\right) \neq \overline{\phi_{0}}\left(k_{i} f_{k_{i}, \alpha_{i}}(y)\right)$. But by $(41)$,

$$
f_{\overline{\phi_{0}}\left(k_{i}\right), \beta_{i}}(z)=\overline{\phi_{0}}\left(k_{i}\right)^{-1} \overline{\phi_{0}}\left(k_{i} f_{k_{i}, \alpha_{i}}(x)\right)=\overline{\phi_{0}}\left(k_{i}\right)^{-1} \overline{\phi_{0}}\left(k_{i} f_{k_{i}, \alpha_{i}}(y)\right) \text {. }
$$

This contradicts the fact that $\overline{\phi_{0}}\left(k_{i} f_{k_{i}, \alpha_{i}}(x)\right) \neq \overline{\phi_{0}}\left(k_{i} f_{k_{i}, \alpha_{i}}(y)\right)$.

\section{The Boundary map And the Tits Bullding}

In view of Step 5 , there exists a continuous bijection $\overline{\phi_{0}}: \hat{X} \rightarrow \hat{X}$ such that $\phi_{0}$ agrees with $\overline{\phi_{0}}$ almost everywhere.

Step 6 ( $\phi_{0}$ is induced by an isometry). If $G$ has no rank one factors, then there exists an element $g \in \operatorname{Isom}(X)$ such that for almost all $x \in \hat{X}, \phi_{0}(x)=g \cdot x$, where - denotes action on the boundary. If $G$ has rank one factors but $\operatorname{rank}(G) \geq 2$, then $\phi_{0}$ is a factor preserving map (possibly composed with a permutation of isomorphic factors).

Note. If we identify $\hat{X}$ with $K / M$, we may push forward the measure $m_{K}$ to obtain a measure $\nu$ on $\hat{X}$. Even though $\nu$ depends on the identification (i.e. on the choice of basepoint), the measure class of $\nu$ is independent of all choices. By "almost all $x \in \hat{X}$ " we mean " $\nu$-almost-everywhere".

Proposition $6.1\left(\bar{\phi}_{0}\right.$ induces a Tits isomorphism $\left.\mathcal{T}(X) \rightarrow \mathcal{T}(X)\right)$. There exists an order preserving bijection $\phi_{G}: \mathcal{T}(X) \rightarrow \mathcal{T}(X)$ such that the restriction of $\phi_{G}$ to $\hat{X}$ agrees with $\overline{\phi_{0}}$ (and hence with $\phi_{0}$ almost everywhere).

Proof of Proposition 6.1. The only properties of $\overline{\phi_{0}}$ used in the proof are that it is a uniformly continuous injective map which is Tits almost everywhere. 
In view of our realization of $\mathcal{T}(X)$, it is enough to show that for any $\sigma \subset \Delta$, there exists $\tau \subset \Delta$ with $|\tau|=|\sigma|$ such that for any $k \in K$,

$$
\overline{\phi_{0}}\left(k K_{\sigma}\right)=\overline{\phi_{0}}(k) K_{\tau} \text {. }
$$

Indeed, if (43) holds, we can define $\phi_{G}\left(k K_{\sigma}\right)=\overline{\phi_{0}}(k) K_{\tau}$; since $\phi_{0}$ is uniformly continuous and injective, $\phi_{G}$ is an order preserving injection. The fact that $\phi_{G}$ is a bijection follows from (43) and invariance of domain (see e.g. [Mu, Theorem 36.5]).

We now prove (43). In particular, we need to show that $\tau$ in (43) (and Step 4) depends only on $\sigma$ and not on $k \in K / K_{\sigma}$. Let $\pi_{\sigma}$ denote the projection $K / M \rightarrow$ $K / K_{\sigma}$. Then $\pi_{\sigma}$ is clearly continuous. Let

$$
E \subset \mathcal{U}_{1}=\left\{k \in \mathcal{U}_{1}: m_{K}\left(k K_{\theta} \cap \mathcal{U}_{1}\right)=m_{K}\left(k K_{\theta}\right) \text { for all } \theta \subset \Delta\right\} .
$$

Then $m_{K}(E)=1$, and thus in particular $E$ is dense. For each $\tau$ with $|\tau|=|\sigma|$, let

$$
F_{\tau}=\left\{z \in K / K_{\sigma}: \overline{\phi_{0}}\left(\pi_{\sigma}^{-1}(z)\right) \subset k^{\prime} K_{\tau} \text { for some } k^{\prime}=k^{\prime}(z)\right\} .
$$

Suppose for now that $\sigma$ is such that $\operatorname{dim} K / K_{\sigma}$ is maximal, i.e. $\operatorname{dim} K / K_{\theta} \leq$ $\operatorname{dim} K / K_{\sigma}$ for all $\theta \subset \Delta$ with $|\theta|=|\sigma|$. Since $\overline{\phi_{0}}$ is continuous and bijective, invariance of domain implies that for every $\tau$ with $F_{\tau}$ nonempty, equality holds in (44), i.e. $\overline{\phi_{0}}\left(\pi_{\sigma}^{-1}(z)\right)=k^{\prime} K_{\tau}$. This also implies that the distinct $F_{\tau}$ are disjoint.

By Step 4, for each $k \in \mathcal{U}_{1}$ there exists $\tau \subset \Delta$ with $|\tau|=|\sigma|$ and $k^{\prime} \in K$ such that $\phi_{0}\left(k K_{\sigma} \cap \mathcal{U}^{\prime}\right) \subset k^{\prime} K_{\tau}$. Thus $E \subset \bigcup_{|\tau|=|\sigma|} \pi_{\sigma}^{-1}\left(F_{\tau}\right) \cap \mathcal{U}_{1}$, and if $x \in E$ and $\pi_{\sigma}(x) \in F_{\tau}$, then $\phi_{0}\left(x K_{\sigma} \cap \mathcal{U}^{\prime}\right) \subset k^{\prime} K_{\tau}$ for some $k^{\prime} \in K$.

We now claim that

$$
\overline{\pi_{\sigma}(E) \cap F_{\tau}} \subset F_{\tau} .
$$

Suppose $\pi_{\sigma}(x) \in \bar{F}_{\tau}$. Let $y \in K$ be any point such that $\pi_{\sigma}(x)=\pi_{\sigma}(y)$. Let $x_{j}$ and $y_{j}$ be two sequences in $E \cap \pi_{\sigma}^{-1}\left(F_{\tau}\right)$ converging to $x$ and $y$ respectively. Since $x_{j} \in E$ and $\lim \pi_{\sigma}\left(x_{j}\right)=\lim \pi_{\sigma}\left(y_{j}\right)$, there exists $y_{j}^{\prime} \in E \cap x_{j} K_{\sigma}$ such that $d\left(y_{j}^{\prime}, y_{j}\right) \rightarrow 0$. Since $\pi_{\sigma}\left(x_{j}\right)=\pi_{\sigma}\left(y_{j}^{\prime}\right) \in F_{\tau}, \pi_{\tau}\left(\overline{\phi_{0}}\left(y_{j}^{\prime}\right)\right)=\pi_{\tau}\left(\overline{\phi_{0}}\left(x_{j}\right)\right)$. Since $\overline{\phi_{0}}$ and $\pi_{\tau}$ are both continuous, $\pi_{\tau}\left(\overline{\phi_{0}}(x)\right)=\lim \pi_{\tau}\left(\overline{\phi_{0}}\left(x_{j}\right)\right)=\lim \pi_{\tau}\left(\overline{\phi_{0}}\left(y_{j}^{\prime}\right)\right)=$ $\lim \pi_{\tau}\left(\overline{\phi_{0}}\left(y_{j}\right)\right)=\pi_{\tau}\left(\overline{\phi_{0}}(y)\right)$. Thus, since $y \in \pi_{\sigma}^{-1}\left(\pi_{\sigma}(x)\right)$ is arbitrary, $\pi_{\sigma}(x) \in F_{\tau}$. This proves (45).

We now claim that each (nonempty) $F_{\tau}$ is open. Indeed, suppose $x \in F_{\tau}$. Let $B_{j}$ be a sequence of balls shrinking to $x$. If all sufficiently small $B_{j}$ contained points in $F_{\tau^{\prime}} \cap \pi_{\sigma}(E)$, where $\tau^{\prime} \neq \tau$, then by (45) we would have $x \in F_{\tau^{\prime}}$, which would contradict the disjointness of the distinct $F_{\tau}$. Hence for some $n, B_{n} \cap \pi_{\sigma}(E) \subset$ $F_{\tau}$. Since $\pi_{\sigma}(E)$ is dense, this implies $B_{n} \subset F_{\tau}$, hence $F_{\tau}$ is open. Now by connectedness, one of the $F_{\tau}$ is all of $K / K_{\sigma}$, and the rest are empty. Hence (43) holds for $\sigma$.

Note that if (43) holds, then $\sigma$ and $\tau$ determine each other. Thus to prove (43) for all $\sigma$ (without the assumption that $K / K_{\sigma}$ has maximal dimension), one can proceed by reverse induction on the dimension of $K / K_{\sigma}$.

Proof of Step 6. It is enough to prove that for all $x$ in $K / M \cong \hat{X}, \overline{\phi_{0}}(x)=g \cdot x$ for some $g \in \operatorname{Isom}(X)$. By Proposition $6.1, \overline{\phi_{0}}$ induces a bijection $\phi_{G}$ of the Tits building associated to $X$ into itself; the map on the cells of maximal dimension (i.e. chambers) is $\overline{\phi_{0}}$, hence a homeomorphism. Thus, $\phi_{G}$ is an order preserving bijection of the Tits building onto itself which is also a homeomorphism of the Furstenberg boundary into itself. By the theorem of Tits ([Ti], see also [Mo, Corollary 16.2 and 
§17]) any such map is induced by an isometry as long as $G$ has no rank one factors, and is factor preserving (up to a permutation) even if $G$ has rank one factors.

\section{THE QUASI-ISOMETRY $\phi$ IS CLOSE TO AN ISOMETRY}

In $\S 7, \S 8$ and $\S 9$ we assume that $G$ has no rank one factors; we will return to the case where $G$ has rank one factors in $\S 10$.

Step 7 ( $\phi$ is close to an isometry). There exists an isometry $g$ and a number $C>$ 0 such that for all $\gamma \in \Gamma, d(\phi(\gamma), \pi(g \gamma))<C$.

We first note a corollary of Step 6:

Corollary 7.1. Let $g \in \operatorname{Isom}(X)$ be as in Step 6. Then there exists a subset $E_{0} \subset G$ with the following properties:

(a) $E_{0}$ has full measure, i.e. for any compact $U \subset G, m\left(E_{0} \cap U\right)=m(U)$ where $m(\cdot)$ denotes Haar measure on $G$.

(b) Let $\mathcal{F}_{0} \subset \mathcal{F}^{*}$ denote the family of flats $\left\{\pi(x A): x \in E_{0}\right\}$. Then, for any $F \in \mathcal{F}_{0}$ and any $x \in \Omega_{F}$, we have $d(\phi(x), g F) \leq N$.

Proof of Corollary 7.1. Let

$E_{0}=\left\{x \in p^{-1}\left(E^{*}\right)\right.$ such that for every chamber $\left.\mathfrak{C} \subset \pi(x A), \phi_{0}(\mathfrak{C})=g \cdot \mathfrak{C}\right\}$

where $E^{*}$ is as in the proof of Lemma 3.2. Then, part (a) holds since the condition $\phi_{0}(\mathfrak{C})=g \cdot \mathfrak{C}$ holds a.e. by Step 6 . The condition (b) holds by Step 3 , since we may identify $F^{\prime}$ as $g F$.

We also note the following structural lemma, analogous to Lemma 3.1:

Lemma 7.2. For every $\delta>0$ there exists a subset $Q \subset K / M \times K / M$ with $m_{K}(Q)>1-\delta$ such that for $\left(k_{1}, k_{2}\right) \in Q$ and $k \in K$ we have $\left(k k_{1}, k k_{2}\right) \in Q$ and for any $h \in G$ the following hold:

(a) The flats $F_{1}=\pi\left(h k_{1} A\right)$ and $F_{2}=\pi\left(h k_{2} A\right)$ satisfy $F_{1} \cap F_{2}=\{\pi(h)\}$.

(b) The flats $F_{1}$ and $F_{2}$ are "almost orthogonal", i.e. there exist constants $\lambda_{1}, \lambda_{2}$ depending only on $X$ and $\delta$, such that if $r>\lambda_{2}$, then

$$
\operatorname{Nbhd}\left(F_{1}, r\right) \cap \operatorname{Nbhd}\left(F_{2}, r\right) \subset B\left(\pi(h), \lambda_{1} r\right) .
$$

Proof. Without loss of generality, we may assume $h=1$. Let

$$
R=\bigcup_{w \in W} \bigcup_{\sigma \subset \Delta} K_{\sigma} w .
$$

We choose $Q$ of the form $\left\{\left(k_{1}, k_{2}\right) \in K \times K: d_{K}\left(k_{1}^{-1} k_{2}, R\right) \geq \nu\right\}$, where $\nu=\nu(\delta, X)$. Suppose $x_{1} \in F_{1}, x_{2} \in F_{2}$. Then we may write $x_{i}=\pi\left(k_{i}^{\prime} a_{i}\right)$ where $a_{i} \in A^{+}$and $k_{i}^{\prime} \in k_{i} W$. Note that for every $\sigma \subset \Delta, d_{K}\left(k_{1}^{\prime}, k_{2}^{\prime} K_{\sigma}\right)>\theta$ where $\theta$ depends only on $\nu$ and $X$.

For $i=1,2$ there exists $\alpha_{i} \in \Delta$ such that $\alpha_{i}\left(x_{i}\right) \succ d\left(x_{i}, e\right)$. Let $\sigma=\left\{\alpha_{1}\right\} \cup\left\{\alpha_{2}\right\}$. Then, by Lemma B.3, if $s=\min _{i=1,2} d\left(x_{i}, e\right)$ is sufficiently large, then $d\left(x_{1}, x_{2}\right) \succ s$. This implies (b).

Proof of Step 7. Let $\mathcal{D} \subset G$ denote a fundamental domain for $\Gamma \backslash G$. For $u \in \mathcal{D}$ let $S_{u}=\{k \in K: p(u k) \in \Omega\}$, where $\Omega$ is as in Step 2 (e). By Step 2 (e) and Fubini's theorem there exists a compact subset $U$ of $\mathcal{D}$ of positive measure such that for any $u \in U, m_{K}\left(S_{u}\right) \geq 1-3|\Sigma| \epsilon$. Then, in view of Lemma 7.2, for any $u \in U, m_{K}\left(Q \cap\left(S_{u} \times S_{u}\right)\right)>0$. 
Now let $\gamma \in \Gamma$ be arbitrary. Let $E_{0}$ be as in Corollary 7.1. In view of Corollary 7.1 (a), we may choose $u \in U$ and $\left(k_{1}, k_{2}\right) \in Q \cap\left(S_{u} \times S_{u}\right)$ so that $\gamma u k_{i} \in E_{0}$ for $i=1,2$. Let $h=\gamma u$ and for $i=1,2$ let $F_{i}=\pi\left(h k_{i} A\right)$. Note that since $U$ is compact, $d(\pi(\gamma), \pi(h))<c$ where $c$ depends only on $U$ (and thus only on $X$ ).

Since $p\left(h k_{i}\right) \in \Omega, \pi(h) \in \Omega_{F_{1}} \cap \Omega_{F_{2}}$. Hence, by Corollary $7.1(\mathrm{~b})$,

$$
\phi(\pi(h)) \subset \operatorname{Nbhd}\left(g F_{1}, N\right) \cap \operatorname{Nbhd}\left(g F_{2}, N\right) .
$$

Since $g$ acts by isometries, and $F_{1}, F_{2}$ are almost orthogonal, the flats $g F_{1}$ and $g F_{2}$ are also almost orthogonal, and intersect at $\pi(g h)=g \pi(h) \in X$. Hence, by Lemma 7.2,

$$
\begin{aligned}
\phi(\pi(h)) & \subset \operatorname{Nbhd}\left(g F_{1}, N\right) \cap \operatorname{Nbhd}\left(g F_{2}, N\right) \subset \operatorname{Nbhd}\left(g F_{1} \cap g F_{2}, N^{\prime}\right) \\
& =\operatorname{Nbhd}\left(\pi(g h), N^{\prime}\right)
\end{aligned}
$$

where $N^{\prime}$ depends only on $N$ and $X$. Since $d(\pi(\gamma), \pi(h))<c, \phi$ is a quasi-isometry and $g$ is an isometry, for any $\gamma \in \Gamma, d(\phi(\gamma), \pi(g \gamma))$ is bounded independently of $\gamma$.

Remark. We actually proved that $d(\phi(\gamma), \pi(g \gamma))$ is bounded by a constant depending only on $\kappa, C, X$, and $\Gamma$.

\section{The Quasi-ISOMEtry $\phi$ is Close to a COMMensuration}

Step 8 ( $\phi$ is close to a commensuration). The element $g$ of Step 7 belongs to $\operatorname{Comm}(\Gamma)$.

Lemma 8.1. Suppose $g \in I \operatorname{som}(X)$ is such that the orbit $p(g \Gamma) \subset \Gamma \backslash I \operatorname{som}(X)$ is bounded. Then $p(g \Gamma)$ is finite, and $g \in \operatorname{Comm}(\Gamma)$.

Proof. This lemma and its equivalence to the statement of Step 8 are due to Nimish Shah, who proved a much more general statement in [Sh]. The proof uses ideas from the theory of unipotent flows. Recall that a finite index subgroup of $\Gamma$ is generated by unipotent elements. By $[\mathrm{Sh}], \overline{p(g) \Gamma}=p(F)$ for a closed subgroup $F$ of $\operatorname{Isom}(X)$ containing $\Gamma$. By Borel density, $F^{0}$ is normal in $G$, and $p\left(F^{0}\right)$ is closed. Hence $F^{0}=G$ or $F^{0}=\{1\}$. Since $p\left(F^{0}\right)$ is compact, $F^{0} \neq G$. Hence $F^{0}$ is the identity, and thus $p(g \Gamma)$ is finite.

Proof of Step 8. This follows immediately from Lemma 8.1. Indeed $\phi: \Gamma \rightarrow \Gamma$ is a quasi-isometry, and by Step 7 there exists $g \in \operatorname{Isom}(X)$ and $c<\infty$ such that for all $\gamma \in \Gamma, d(\pi(g \gamma), \phi(\gamma))<c$. Since $\phi(\gamma) \in \Gamma$, we get $d(p(g \gamma), p(\Gamma))<c$. Hence the orbit $p(g \Gamma)$ is bounded in $\Gamma \backslash \operatorname{Isom}(X)$. By Lemma 8.1 this implies $g \in \operatorname{Comm}(\Gamma)$.

This completes the proof of Theorem 0.1 .

\section{Proof of Corollary 0.2}

This is by now standard; the relevant argument appeared in [CC], [S1], [FS], and [S2]. We give only a short outline for completeness. Suppose $\Lambda$ is finitely generated, and we have a $(\kappa, c)$ quasi-isometry $q: \Lambda \rightarrow \Gamma$. For each $\lambda \in \Lambda$, left multiplication by $\lambda$ is an isometry $\ell_{\lambda}: \Lambda \rightarrow \Lambda$; so $q \circ \ell_{\lambda} \circ q^{-1}$ is a quasi-isometry from $\Gamma$ to $\Gamma$. Thus we have a map $\Psi: \Lambda \rightarrow Q I(\Gamma)$ sending $\lambda$ to $q \circ \ell_{\lambda} \circ q^{-1}$, which one can verify to be a homomorphism. By Theorem 0.1 there is an isomorphism $i$ of $Q I(\Gamma)$ with $\operatorname{Comm}(\Gamma)$. Let $\Phi=i \circ \Psi$. Thus $\Phi(\Lambda)$ is a subgroup of $\operatorname{Comm}(\Gamma)$. 
The fact that $\Lambda$ is finitely generated and the definition of $\Phi$ imply that for any $r \gg 1$, the set $\{\lambda \in \Lambda: d(\Phi(\lambda) 1,1)<r\}$ is finite. Hence the kernel of $\Phi$ is finite, and also $\Phi(\Lambda)$ is discrete.

To show $\Phi(\Lambda)$ is a lattice, one may argue as follows. By the construction, uniformly for $\gamma \in \Gamma, d\left(\gamma, \Psi\left(q^{-1}(\gamma)\right) 1\right)<\infty$.

Note that for any $\lambda \in \Lambda, \Psi(\lambda)$ is a $(\kappa, C)$ quasi-isometry $\Gamma \rightarrow \Gamma$, with constants $\kappa$ and $C$ which are independent of $\lambda$. Hence, since our bound in Theorem 0.1 depended only on $\Gamma$ and the quasi-isometry constants, $d(\Psi(\lambda), \Phi(\lambda))<\infty$ uniformly for $\lambda \in \Lambda$. Hence, uniformly for $\gamma \in \Gamma, d\left(\gamma, \Phi\left(q^{-1}(\gamma)\right) 1\right)<\infty$, i.e. every point $\gamma \in \Gamma$ is within a finite distance from a point in $\Phi(\Lambda)$. Hence $\Phi(\Lambda)$ has finite co-volume and is thus a lattice.

Hence $\Phi(\Lambda)$ is a lattice which is a subgroup of $\operatorname{Comm}(\Gamma)$. It is well known that any such lattice is commensurable to $\Gamma$; this is a consequence of the totally disconnected case of Margulis superrigidity and is a step in the proof of arithmeticity. See e.g. [Zim, pp. 120-121] for the details.

\section{RANK ONE FACTORS}

In this section we prove the following:

Proposition 10.1 ( $\phi$ extends to all of $G$ and is factor preserving). Let $G$ be $a$ semisimple Lie group with finite center and rank at least 2; suppose $G=G_{1} \times$ $G_{2} \times \cdots \times G_{n}$ where each $G_{i}$ is simple. Let $\Gamma$ be an irreducible nonuniform lattice in $G$, and let $\phi$ be a quasi-isometry of $\Gamma$ to itself, in the word metric. Then for each $i$ there exists a quasi-isometry $\psi_{i}: G_{i} \rightarrow G_{i}$ (defined on all of $G_{i}$ ) such that $\phi$ is a bounded distance from the product map $\psi_{1} \times \psi_{2} \times \cdots \times \psi_{n}$ (possibly composed with an isometry permuting isomorphic factors). If $G_{i}$ is any factor with $\operatorname{rank} G_{i} \geq 2$, then $\psi_{i}$ can be taken to be an isometry.

This is an intermediate result which is intended to be combined with results from [S1], [S2] and [FS] to give a generalization of Theorem 0.1 and Corollary 0.2 to the case where $\operatorname{rank} G \geq 2$ but $G$ has rank one factors; see [Fa] for an outline of the argument. The proof of Proposition 10.1 is a variation on the argument in $\S 7$. We begin with the following:

Lemma 10.2. Let $G$ be as in Proposition 10.1. Fix $i, 1 \leq i \leq n$, and let $\pi_{i}$ denote the projection $G \rightarrow G_{i} / K_{i}$, where $K_{i}$ is the maximal compact subgroup of $G_{i}$. Let $\mathcal{C}$ be a compact subset of $\Gamma \backslash G$. Suppose $x \in p^{-1}(\mathcal{C}), y \in p^{-1}(\mathcal{C})$, and $\pi_{i}(x)=\pi_{i}(y)$. Then there exist flats $F_{1}, F_{2}, H_{1}, H_{2}$ and points $x_{0}, y_{0} \in G$ such that the following hold:

(a) $d\left(\pi(x), \pi\left(x_{0}\right)\right)<c_{0}$ and $d\left(\pi(y), \pi\left(y_{0}\right)\right)<c_{0}$ where $c_{0}$ is independent of $x$ and $y$.

(b) $\left\{\pi\left(x_{0}\right)\right\}=F_{1} \cap F_{2}$ and $\left\{\pi\left(y_{0}\right)\right\}=H_{1} \cap H_{2}$.

(c) $F_{1}$ and $F_{2}$ are almost orthogonal (see Lemma 7.2); so are $H_{1}$ and $H_{2}$.

(d) $\pi\left(x_{0}\right) \in \Omega_{F_{1}} \cap \Omega_{F_{2}}$ and $\pi\left(y_{0}\right) \in \Omega_{H_{1}} \cap \Omega_{H_{2}}$, where sets of the type $\Omega_{F}$ are as in Step 3.

(e) For every chamber $\mathfrak{C}$ contained in $F_{1}$ or $F_{2}$ or $H_{1}$ or $H_{2}, \phi_{0}(\mathfrak{C})=\overline{\phi_{0}}(\mathfrak{C})$.

(f) $\pi_{i}\left(F_{1}\right)=\pi_{i}\left(H_{1}\right)$ and $\pi_{i}\left(F_{2}\right)=\pi_{i}\left(H_{2}\right)$.

Proof of Lemma 10.2. Let $E_{0}$ be as in the proof of Corollary 7.1, i.e.

$E_{0}=\left\{x \in p^{-1}\left(E^{*}\right)\right.$ such that for every chamber $\left.\mathfrak{C} \subset \pi(x A), \phi_{0}(\mathfrak{C})=\overline{\phi_{0}}(\mathfrak{C})\right\}$ 
where $E^{*}$ is as in the proof of Lemma 3.2. Then $E_{0}$ has full measure. Let $E_{0}^{\prime}=$ $\left\{x \in G\right.$ such that $x k \in E_{0}$ for almost all $\left.k \in K\right\}$. Then $E_{0}^{\prime}$ also has full measure.

Let $\mathcal{D}$ be a fundamental domain for $\Gamma \backslash G$. Let $U \subset \mathcal{D}$ be as in the proof of Step 7 . Since $x, y \in p^{-1}(\mathcal{C}), \mathcal{C}$ and $U$ are compact, and $E_{0}^{\prime}$ has full measure, there exists $z \in G$ with $d(\pi(z), e)<c_{0}, x z \in p^{-1}(U) \cap E_{0}^{\prime}$ and $y z \in p^{-1}(U) \cap E_{0}^{\prime}$. Let $x_{0}=x z$ and let $y_{0}=y z$.

Let $Q \subset K / M \times K / M$ be as in Lemma 7.2. For $u \in U$ let $S_{u} \subset K / M$ be as in the proof of Step 7. By an argument identical to the one in $\S 7$,

$$
m_{K}\left(Q \cap\left(S_{p\left(x_{0}\right)} \times S_{p\left(x_{0}\right)}\right) \cap\left(S_{p\left(y_{0}\right)} \times S_{p\left(y_{0}\right)}\right)\right)>0 .
$$

Hence there exist $\left(k_{1}, k_{2}\right) \in K / M \times K / M$ such that $\left(k_{1}, k_{2}\right) \in Q \cap\left(S_{p\left(x_{0}\right)} \times S_{p\left(x_{0}\right)}\right) \cap$ $\left(S_{p\left(y_{0}\right)} \times S_{p\left(y_{0}\right)}\right)$ and for $j=1,2, x_{0} k_{j} \in E_{0}$ and $y_{0} k_{j} \in E_{0}$. By construction, the flats $F_{j}=\pi\left(x_{0} k_{j} A\right)$ and $H_{j}=\pi\left(y_{0} k_{j} A\right)$ satisfy the conditions of the lemma.

Lemma 10.3. Let $G$ be as in Proposition 10.1. Fix $i, 1 \leq i \leq n$, and let $\pi_{i}(\cdot)$ and $\mathcal{C}$ be as in Lemma 10.2. Then, if $x \in p^{-1}(\mathcal{C}), y \in p^{-1}(\overline{\mathcal{C}})$, and $\pi_{i}(x)=\pi_{i}(y)$, $d\left(\pi_{i}(\phi(x)), \pi_{i}(\phi(y))\right)<C$, where $C$ does not depend on $x$ and $y$.

Proof of Lemma 10.3. Let $x_{0}, y_{0}, F_{1}, F_{2}, H_{1}$ and $H_{2}$ be as in Lemma 10.2. By Step 3, for $j=1,2$ there exist flats $F_{j}^{\prime}, H_{j}^{\prime}$ such that $\phi\left(\Omega_{F_{j}}\right) \subset \operatorname{Nbhd}\left(F_{j}^{\prime}, N\right)$ and $\phi\left(\Omega_{H_{j}}\right) \subset \operatorname{Nbhd}\left(H_{j}^{\prime}, N\right)$. Hence $\phi\left(x_{0}\right) \subset \operatorname{Nbhd}\left(F_{1}^{\prime}, N\right) \cap \operatorname{Nbhd}\left(F_{2}^{\prime}, N\right)$ and $\phi\left(y_{0}\right) \subset \operatorname{Nbhd}\left(H_{1}^{\prime}, N\right) \cap \operatorname{Nbhd}\left(H_{2}^{\prime}, N\right)$.

Note that since $F_{1}, F_{2}$ and $H_{1}, H_{2}$ are almost orthogonal, there exist points $x^{\prime}, y^{\prime}$ and a constant $C^{\prime}$ independent of $x$ and $y$ such that $\operatorname{Nbhd}\left(F_{1}^{\prime}, N\right) \cap \operatorname{Nbhd}\left(F_{2}^{\prime}, N\right) \subset$ $\operatorname{Nbhd}\left(x^{\prime}, C^{\prime}\right)$ and $\operatorname{Nbhd}\left(H_{1}^{\prime}, N\right) \cap \operatorname{Nbhd}\left(H_{2}^{\prime}, N\right) \subset \operatorname{Nbhd}\left(y^{\prime}, C^{\prime}\right)$. Thus $d\left(\phi\left(x_{0}\right), x^{\prime}\right)<$ $C^{\prime \prime}$, and $d\left(\phi\left(y_{0}\right), y^{\prime}\right)<C^{\prime \prime}$, where $C^{\prime \prime}$ does not depend on $x$ and $y$.

Since $\pi_{i}\left(F_{1}\right)=\pi_{i}\left(H_{1}\right)$ and $\pi_{i}\left(F_{2}\right)=\pi_{i}\left(H_{2}\right)$, by Step 6 we have $\pi_{i}\left(F_{1}^{\prime}\right)=\pi_{i}\left(H_{1}^{\prime}\right)$ and $\pi_{i}\left(F_{2}^{\prime}\right)=\pi_{i}\left(H_{2}^{\prime}\right)$. Hence $d\left(\pi_{i}(\phi(x)), \pi_{i}(\phi(y))\right)<C$ as required.

Proof of Proposition 10.1. Since $\Gamma$ is irreducible, $\pi_{i}\left(p^{-1}(\mathcal{C})\right)=G_{i} / K_{i}$. If $u \in$ $G_{i} / K_{i}$, choose $x \in \pi_{i}^{-1}(u) \cap p^{-1}(\mathcal{C})$, and let $\psi_{i}(u)=\pi_{i}(\phi(x))$. In view of Lemma 10.3 , this (up to a fixed additive constant) does not depend on the choice of $x$. To see that $\psi_{i}$ is a quasi-isometry, we may argue as follows: suppose $u_{1}, u_{2} \in G_{i} / K_{i}$. It follows, e.g. from the Moore ergodicity theorem applied to the action of the Cartan subgroup of $\prod_{j \neq i} G_{j}$ on $\Gamma \backslash G$ that there exist points $x_{1}, x_{2} \in p^{-1}(\mathcal{C})$ such that $\pi_{i}\left(x_{1}\right)=u_{1}, \pi_{i}\left(x_{2}\right)=u_{2}$, and $\pi_{j}\left(x_{1}\right)=\pi_{j}\left(x_{2}\right)$ for $j \neq i$. Note that $d\left(x_{1}, x_{2}\right)=$ $d\left(u_{1}, u_{2}\right)$, and by Lemma 10.3, $\left|d\left(\psi_{i}\left(u_{1}\right), \psi_{i}\left(u_{2}\right)\right)-d\left(\phi\left(x_{1}\right), \phi\left(x_{2}\right)\right)\right|<C^{\prime \prime}$ where $C^{\prime \prime}$ is fixed. Since $\phi$ is a quasi-isometry, this implies that $\psi_{i}$ is also.

\section{Appendix A. Quasi-ISOmetries}

A.1. The packing lemma and its corollaries. The following lemma is a coarse version of invariance of domain. The proof given in $[\mathrm{EF}, \S 8.2]$ is a variation of an argument due to Geoff Mess ${ }^{1}$. See also [FS, Corollary 5.3].

Lemma A.1 (Local packing). Let $q: B \rightarrow \mathbb{R}^{n}$ be a continuous map which is a $(\kappa, C)$ quasi-isometric embedding from the ball $B=B\left(x, r_{1}\right) \subset \mathbb{R}^{n}$ into $\mathbb{R}^{n}$. Then there exist constants $\eta_{1}>1, \eta_{2}>1$ depending only on $\kappa$ such that for any $r<r_{1}$,

$$
\operatorname{Nbhd}\left(q(B), \eta_{1} C\right) \supset B\left(q(x), r / \eta_{2}\right)
$$

(i.e. $q(B)$ essentially contains the ball $B\left(q(x), r / \eta_{2}\right)$ ).

\footnotetext{
${ }^{1}$ Thanks to Shmuel Weinberger for telling us about this argument.
} 
Corollary A.2 (Packing of metric interiors). Let $U, V$ be subsets of $\mathbb{R}^{n}$ and let $q$ : $U \rightarrow \mathbb{R}^{n}$ be a $(\kappa, C)$ quasi-isometric embedding. Suppose there exists $R>0$ with $\operatorname{Int}(V, R)$ path connected and $q^{-1}(\operatorname{Int}(V, R))$ a nonempty subset of $\operatorname{Int}\left(U, 4 \eta_{1} \eta_{2} C\right)$. Then $\operatorname{Int}(V, R) \subset \operatorname{Nbhd}\left(q(U), \eta_{1} C\right)$.

Proof. Let $W=\operatorname{Nbhd}\left(q(U), \eta_{1} C\right)$. Take any $y \in q(U) \cap \operatorname{Int}(V, R)$. Let $r_{1}=4 \eta_{1} \eta_{2} C$. By assumption, $q^{-1}(y) \in \operatorname{Int}\left(U, r_{1}\right)$, hence by Lemma A.1, $W \supset B\left(y, 3 \eta_{1} C\right)$. Since $W \cap \operatorname{Int}(V, R)$ is nonempty and $\operatorname{Int}(V, R)$ is connected, this implies that $W \supset$ $\operatorname{Int}(V, R)$.

Lemma A.3 (Packing of complexes). Let $\mathcal{P}_{1}, \ldots, \mathcal{P}_{m}$ be isometrically embedded $d$ dimensional convex Euclidean polyhedra in $X$. Let $B$ be a ball in $X$, and suppose $q: \mathbb{R}^{d} \rightarrow X$ is a $(\kappa, C)$ quasi-isometric embedding such that

$$
q\left(\mathbb{R}^{d}\right) \cap B \subset \bigcup_{i=1}^{m} \mathcal{P}_{i} .
$$

Suppose the $\mathcal{P}_{i}$ "come close only near their boundaries", i.e. there exists a constant $r>\left(4 \kappa \eta_{1} \eta_{2}+1\right) C$ (where $\eta_{1}, \eta_{2}$ are as in Lemma A.1) such that for all $i \neq j$,

$$
\operatorname{Int}\left(\mathcal{P}_{i}, r\right) \cap \operatorname{Nbhd}\left(\mathcal{P}_{j},\left(4 \kappa \eta_{1} \eta_{2}+1\right) C\right)=\emptyset .
$$

Suppose there is a constant $\lambda^{\prime \prime}$ such that for all $i$,

$$
h d\left(\operatorname{Int}\left(\mathcal{P}_{i}, r\right), \mathcal{P}_{i}\right) \leq \lambda^{\prime \prime} r .
$$

Then there exists a subset $\sigma$ of $\{1, \ldots, m\}$ and a constant $\lambda^{\prime}$ (depending only on $\lambda^{\prime \prime}, \kappa, m$ and the number faces of the $\mathcal{P}_{i}$ ) such that

$$
h d\left(q\left(\mathbb{R}^{d}\right) \cap B^{\prime}, \bigcup_{i \in \sigma} \mathcal{P}_{i} \cap B^{\prime}\right) \leq \lambda^{\prime} r
$$

where $B^{\prime}=\operatorname{Int}\left(B, \lambda^{\prime} r\right)$.

Proof. Let $V_{j}=\mathcal{P}_{j} \cap B$, and let $\sigma=\left\{j \in[1, m]: q\left(\mathbb{R}^{d}\right) \cap \operatorname{Int}\left(V_{j}, r\right) \neq \emptyset\right\}$. By convexity $V_{j}$ is connected for every $j \in \sigma$.

For $j \in \sigma$, let $U_{j}=q^{-1}\left(V_{j}\right)$. Now suppose $x \in q^{-1}\left(\operatorname{Int}\left(V_{j}, r\right)\right)$. Then

$$
q\left(B\left(x, 4 \eta_{1} \eta_{2} C\right)\right) \subset B\left(q(x),\left(4 \kappa \eta_{1} \eta_{2}+1\right) C\right) \cap q\left(\mathbb{R}^{d}\right) \cap B \subset V_{j}
$$

by (46). Hence $q^{-1}\left(\operatorname{Int}\left(V_{j}, r\right)\right) \subset \operatorname{Int}\left(U_{j}, 4 \eta_{1} \eta_{2} C\right)$, and thus by Corollary A.2, $\operatorname{Int}\left(V_{j}, r\right) \subset \operatorname{Nbhd}\left(q\left(U_{j}\right), \eta_{1} C\right)$. Since $q\left(U_{j}\right) \subset V_{j}$ and using $(47), h d\left(q\left(U_{j}\right), V_{j}\right) \leq$ $\lambda^{\prime \prime} r$. Hence

$$
\bigcup_{j \in \sigma} V_{j} \subset \operatorname{Nbhd}\left(q\left(\mathbb{R}^{d}\right), \lambda^{\prime \prime} r\right)
$$

Suppose $\rho \gg C$, and $x \in q\left(\mathbb{R}^{d}\right) \cap \operatorname{Int}(B, \rho)$. Then, since $q\left(B\left(q^{-1}(x), \rho /(2 \kappa)\right)\right) \subset$ $q\left(\mathbb{R}^{d}\right) \cap B(x, \rho)$ and using again Lemma A.1, there exists a constant $\nu$ depending only on $\kappa$, and $d$ such that

$$
\operatorname{Vol}_{d}\left(q\left(\mathbb{R}^{d}\right) \cap B(x, \rho)\right) \geq \nu \rho^{d}
$$

where $\mathrm{Vol}_{d}$ denotes the $d$-dimensional volume.

Now suppose there exists $x \in q\left(\mathbb{R}^{d}\right)$ such that $x \in B^{\prime}$, but $d\left(x, \bigcup_{j \in \sigma} V_{j}\right) \geq \rho$. Then, $q\left(\mathbb{R}^{d}\right) \cap B(x, \rho) \subset \bigcup_{j \notin \sigma} V_{j}$. But then, since $q\left(\mathbb{R}^{d}\right) \cap \operatorname{Int}\left(V_{j}, r\right)=\emptyset$ for $j \notin \sigma$, 
$\operatorname{Vol}_{d}\left(q\left(\mathbb{R}^{d}\right) \cap B(x, \rho)\right) \leq \lambda^{\prime \prime} r \rho^{d-1}$, where $\lambda^{\prime \prime}$ depends only on $m, d$, and the number of faces of the $\mathcal{P}_{i}$. When $\rho>\left(\lambda^{\prime \prime} / \nu\right) r$, this contradicts (49). Let $\lambda^{\prime}=\lambda^{\prime \prime} / \nu$. Then,

$$
q\left(\mathbb{R}^{d}\right) \cap B^{\prime} \subset \operatorname{Nbhd}\left(\bigcup_{j \in \sigma} V_{j}, \lambda^{\prime} r\right) .
$$

The estimates (48) and (50) imply the lemma.

\section{A.2. Closeness sets and intersections.}

Lemma A.4 (Quasi-isometries preserve closeness sets). Suppose $X$ and $Y$ are metric spaces, $q: X \rightarrow Y$ is a $(\kappa, C)$ quasi-isometric embedding, $U$ and $V$ are subsets of $X$, and $U^{\prime}, V^{\prime}$ are subsets of $Y$.

(i) Suppose $q(U) \subset U^{\prime}, q(V) \subset \operatorname{Nbhd}\left(V^{\prime}, C^{\prime}\right)$, and $r>C^{\prime}>C$. Then

$$
q(U \cap \operatorname{Nbhd}(V, r)) \subset U^{\prime} \cap \operatorname{Nbhd}\left(V^{\prime}, 3 \kappa r\right) .
$$

(ii) Suppose $h d\left(q(U), U^{\prime}\right)<C^{\prime}, h d\left(q(V), V^{\prime}\right)<C^{\prime}$ and $r>3 \kappa C^{\prime}>3 \kappa C$. Suppose also that there exists a constant $\lambda$ such that

$$
\begin{aligned}
& U^{\prime} \cap \operatorname{Nbhd}\left(V^{\prime}, 3 \kappa r\right) \subset \operatorname{Nbhd}\left(U^{\prime} \cap V^{\prime}, \lambda r\right) \text { and } \\
& \qquad U \cap \operatorname{Nbhd}(V, 3 \kappa r) \subset \operatorname{Nbhd}(U \cap V, \lambda r) .
\end{aligned}
$$

Then,

$$
h d\left(q(U \cap V), U^{\prime} \cap V^{\prime}\right)<\lambda^{\prime} r
$$

where $\lambda^{\prime}$ depends only on $\kappa$ and $\lambda$.

Proof. Suppose $u \in U \cap \operatorname{Nbhd}(V, r)$; then there exists $v \in V$ with $d(u, v) \leq r$. Since $r>C$, by $(1), d(q(u), q(v)) \leq 2 \kappa r$. By assumption, there exists $v^{\prime} \in V^{\prime}$ such that $d\left(q(v), v^{\prime}\right) \leq C^{\prime}$. Then, $d\left(q(u), v^{\prime}\right) \leq 2 \kappa r+C^{\prime} \leq 3 \kappa r$, hence $q(u) \in$ $U^{\prime} \cap \operatorname{Nbhd}\left(V^{\prime}, 3 \kappa r\right)$.

To get (ii) we first modify $q$ by $O\left(C^{\prime}\right)$ so that $q(U) \subset U^{\prime}$. Then by part (i),

$$
q(U \cap V) \subset q(U \cap \operatorname{Nbhd}(V, r)) \subset U^{\prime} \cap \operatorname{Nbhd}\left(V^{\prime}, 3 \kappa r\right) .
$$

Hence, $q(U \cap V) \subset \operatorname{Nbhd}\left(U^{\prime} \cap V^{\prime}, \lambda r\right)$. Applying the same argument to the coarse inverse of $q$ shows that $U^{\prime} \cap V^{\prime} \subset \operatorname{Nbhd}\left(q(U \cap V), \lambda^{\prime} r\right)$.

\section{Appendix B. Geometric estimates}

B.1. Geometry of neighboring flats. In this subsection we prove the following lemma:

Lemma B.1 (Geometry of neighboring flats; cf. [Mo], Theorem 7.8). (i) There exist constants $\lambda_{0}, \lambda_{1}, \lambda_{2}$ depending only on $X$ such that the following holds: For each pair of flats $F_{1}, F_{2} \subset X$ there exist constants $c_{\alpha} \geq 0$ such that if we denote

$$
\mathcal{P}(r)=\left\{x \in F_{1} \quad: \quad c_{\alpha} e^{\alpha(x)} \leq e^{r}, \quad \forall \alpha \in \Sigma\right\},
$$

then for $r>\lambda_{0}\left(1+d\left(F_{1}, F_{2}\right)\right)$, we have $\mathcal{P}\left(\lambda_{1} r\right) \subset F_{1} \cap \operatorname{Nbhd}\left(F_{2}, r\right) \subset$ $\mathcal{P}\left(\lambda_{2} r\right)$. (Note that if $c_{\alpha}=0$, then the inequality (52) is always satisfied). In particular, there exists a convex polyhedron $\mathcal{P} \subset F_{1}$ such that $h d\left(F_{1} \cap \operatorname{Nbhd}\left(F_{2}, r\right), \mathcal{P}\right)=O(r)$. 
(ii) Let $L_{1}$ be a hyperplane in $F_{1}$. Then there exists a hyperplane $L_{2} \subset F_{2}$ such that for all $r>\lambda_{0}\left(1+d\left(F_{1}, F_{2}\right)\right)$, we have $F_{2} \cap \operatorname{Nbhd}\left(L_{1}, r\right) \subset \operatorname{Nbhd}\left(L_{2}, \lambda r\right)$ where $\lambda$ depends only on $X$.

(iii) Let $\mathcal{P}_{1}$ be a (possibly unbounded) polyhedron in $F_{1}$, and $r>\lambda_{0}\left(1+d\left(F_{1}, F_{2}\right)\right)$. Then there exists a polyhedron $\mathcal{P}_{2} \subset F_{2}$ such that $h d\left(F_{2} \cap \operatorname{Nbhd}\left(\mathcal{P}_{1}, r\right), \mathcal{P}_{2}\right) \leq$ $\lambda r$, where $\lambda$ depends only on $X$.

The proof of Lemma B.1 uses the following:

Lemma B.2 (Gaussian elimination). Let $M A N$ denote the Langlands decomposition of a Borel subgroup $B$ where $M$ is compact. Let $W=N_{K}(A) / M$ denote the Weyl group associated to $(G, A)$, let $w_{0}$ denote the longest element of $W$ and let $\bar{N}$ denote $w_{0} N w_{0}^{-1}$.

For every compact set $\mathcal{C} \subset G$, there exist compact sets $U_{N} \subset N, U_{A} \subset A$, and $U_{\bar{N}} \subset \bar{N}$ such that $\mathcal{C} \subset W U_{\bar{N}} M U_{A} U_{N}$. The set $U_{N}$ can be chosen so that $a^{-1} U_{N} a \subseteq U_{N}$ for $a \in \overline{A_{+}}$.

Proof of Lemma B.2. Note that for $w \in W, w^{-1} B w B \subset w_{0}^{-1} B w_{0} B$. Then, for $w \in W, B w B=w\left(w^{-1} B w\right) B \subset w\left(w_{0}^{-1} B w_{0}\right) B$. Hence, using the Bruhat decomposition,

$$
G=\bigcup_{w \in W} w\left(w_{0}^{-1} B w_{0}\right) B
$$

Now for $g$ in $\left(w_{0}^{-1} B w_{0}\right) B$, we can write $g=\bar{n}$ man, where $\bar{n} \in \bar{N}, m \in M, a \in A$, $n \in N$, and the functions $g \rightarrow \bar{n}(g)$ etc. are clearly continuous (e.g. one can see this by embedding in $S L(n, \mathbb{R})$ ). Thus these functions are uniformly continuous on any compact set in the interior of $\left(w_{0}^{-1} B w_{0}\right) B$. This, together with (53) implies the lemma.

We remark that the $S L(n, \mathbb{R})$ case of this lemma is the well-known "complete pivoting" algorithm for inverting a matrix. In this case $N$ is the set of upper triangular matrices with 1's along the main diagonal, $\bar{N}$ is the set of lower triangular matrices with 1's along the main diagonal, $A$ is the set of diagonal matrices of determinant 1 and $M$ is the finite group of diagonal matrices with \pm 1 along the diagonal (and determinant 1). Thus (after a suitable permutation corresponding to $W$ ) one must factor a matrix into a product of an upper triangular and a lower triangular matrix; this is done by Gaussian elimination, except that one always performs row exchanges in order to divide by as large a number as possible.

Proof of Lemma B.1. Without loss of generality, we may assume $F_{2}=\pi(A)$, and that there exists a point $\pi(g)$ on $F_{1}$ such that $F_{1}=\pi(g A)$, and $d(\pi(g), e)<$ $d\left(F_{1}, F_{2}\right)+1$. In the sequel we abuse notation by writing for $g_{1}, g_{2} \in G, d\left(g_{1}, g_{2}\right)$ instead of $d\left(\pi\left(g_{1}\right), \pi\left(g_{2}\right)\right)$.

We first prove the following inequality: there exist $w \in W$ and a constant $\lambda$ depending only on $X$ such that for any $a \in A^{+}$

$$
\lambda^{-1} d(w a, g a)-O(d(g, 1)) \leq d\left(F_{2}, \pi(g a)\right) \leq \lambda d(w a, g a)+O(d(g, 1))
$$

(all the implied constants depend only on $X$ ). This is proved as follows. Using the Iwasawa decomposition, we may write $g=k a_{1} n_{1}$ where $k \in K, a_{1} \in A, n_{1} \in N$. Then

$$
d(g, 1)=d\left(a_{1} n_{1}, 1\right)=d\left(n_{1}, a_{1}^{-1}\right) .
$$


But $d\left(n_{1}, a_{1}^{-1}\right) \geq d\left(n_{1}, A\right) \geq \lambda^{-1} d\left(n_{1}, 1\right)$ where the last inequality uses [EF, Lemma A.3] (or [Mo, Lemma 7.6]). Hence

$$
d\left(n_{1}, 1\right) \leq \lambda d(g, 1)
$$

Then from (55) and (56),

$$
d\left(a_{1}, 1\right)=d\left(1, a_{1}^{-1}\right) \leq d\left(1, n_{1}\right)+d\left(n_{1}, a_{1}^{-1}\right) \leq(\lambda+1) d(g, 1) .
$$

Then using Lemma B.2 we may write $k=w \bar{n} m a_{2} n_{2}$ where $w \in W, \bar{n} \in \bar{N}, m \in M$, $a_{2} \in A, n_{2} \in N$, where $d\left(a_{2}, 1\right)=O(1)$ and $d\left(n_{2}, 1\right)=O(1)$. Then, $g=w \bar{n} m a_{3} n_{3}$, where $a_{3}=a_{2} a_{1} \in A$ and $n_{3}=\left(a_{1}^{-1} n_{2} a_{1}\right) n_{1} \in N$. By (57) and the triangle inequality, $d\left(a_{3}, 1\right)=O(d(g, 1))$. Also by (57), (56) and the triangle inequality, $d\left(n_{3}, 1\right)=O(d(g, 1))$. Thus,

$$
\begin{array}{rlr}
d(A, g a) & =d\left(A, w \bar{n} m a_{3} n_{3} a\right) \\
& =d\left(A, w a\left(a^{-1} \bar{n} a\right)\left(m a_{3} a^{-1} n_{3} a\right)\right) \\
& =d\left(a^{-1} w^{-1} A,\left(a^{-1} \bar{n} a\right)\left(m a_{3} a^{-1} n_{3} a\right)\right) & \\
& =d\left(A,\left(a^{-1} \bar{n} a\right)\left(m a_{3} a^{-1} n_{3} a\right)\right) & \\
& =d\left(A, a^{-1} \bar{n} a\right)+O\left(d\left(m a_{3} a^{-1} n_{3} a, 1\right)\right) & \text { since } w A w^{-1}=A \text { and } a \in A \\
& =d\left(A, a^{-1} \bar{n} a\right)+O(d(g, 1))
\end{array}
$$

where in the last line we used $d\left(n_{3}, 1\right)=O(d(g, 1)), d\left(a_{3}, 1\right)=O(d(g, 1))$ and the fact that conjugation by $a$ is nonexpanding on $N$. Similarly,

$$
\begin{aligned}
d(w a, g a) & =d\left(w a, w \bar{n} m a_{3} n_{3} a\right)=d\left(w a, w a\left(a^{-1} \bar{n} a\right)\left(m a_{3} a^{-1} n_{3} a\right)\right) \\
& =d\left(1, a^{-1} \bar{n} a\right)+O(d(g, 1)) .
\end{aligned}
$$

But by [EF, Lemma A.3] (or [Mo, Lemma 7.6]), for $\bar{n}^{\prime} \in \bar{N}, \lambda^{-1} d\left(1, \bar{n}^{\prime}\right) \leq d\left(A, \bar{n}^{\prime}\right) \leq$ $\lambda d\left(1, \bar{n}^{\prime}\right)$. This, together with (58) and (59) implies (54).

Recall that $W$ acts transitively on the Weyl chambers of $A$ based at $e$. Hence any chamber based at $g$ of $F_{1}$ is of the form $\mathfrak{C}_{w}=\pi\left(g w \overline{A_{+}} w^{-1}\right)$ for some $w \in W$. Then by (54), for each chamber $\mathfrak{C}_{w}$ of $F_{1}$ based at $\pi(g)$, there exists $w^{\prime} \in W$ such that

$$
d\left(w^{\prime} a, g w a\right)=O(r), \quad \text { when } \quad \pi(g w a) \in \mathfrak{C}_{w} \cap \operatorname{Nbhd}\left(F_{2}, r\right) .
$$

Thus on $\mathfrak{C}_{w} \cap \operatorname{Nbhd}\left(F_{2}, r\right)$, the map $\psi_{w^{\prime}}: F_{1} \rightarrow F_{2}$ given by $\psi_{w^{\prime}}(\pi(g w a))=\pi\left(w^{\prime} a\right)$ moves each point by at most $O(r)$. Putting these together we get a map $\psi$ : $F_{1} \cap \operatorname{Nbhd}\left(F_{2}, r\right) \rightarrow F_{2}$ which is "coarsely the identity", i.e. it moves each point by at most $O(r)$. This implies that the $w^{\prime}$ can be chosen in a consistent way, i.e. there exists $w^{\prime \prime} \in W$ such that

$$
d(\psi(\pi(g a)), \pi(g a))=d\left(w^{\prime \prime} a, g a\right)=O(r), \quad \text { when } \quad \pi(g a) \in F_{1} \cap \operatorname{Nbhd}\left(F_{2}, r\right) .
$$

The equation (60) implies (ii), since we may take $L_{2}=\psi\left(L_{1}\right)$.

We now prove (i). In view of (60),

$$
d\left(w^{\prime \prime} a, g a\right)=O(r) \quad \text { if and only if } \quad d(A, g a)=O(r) .
$$


First we show that for each $w \in W$ and each $\alpha \in w \Sigma^{+}$, there exists a constant $c_{\alpha, w} \geq 0$ such that if $\mathcal{P}_{w}(r)=\left\{x \in \mathfrak{C}_{w} \quad: c_{\alpha, w} e^{\alpha(x)} \leq e^{r}\right\}$ for all $\alpha \in w \Sigma^{+}$, then

$$
\mathcal{P}_{w}\left(\lambda_{1}^{\prime \prime} r\right) \subset \mathfrak{C}_{w} \cap \operatorname{Nbhd}\left(F_{2}, r\right) \subset \mathcal{P}_{w}\left(\lambda_{2}^{\prime \prime} r\right) .
$$

To prove (62) we may assume $w=1$ (to handle the other Weyl chambers in $F_{1}$, we may replace $g$ by $g w$ for a suitable $w \in W)$. We use (58), for $a \in \overline{A_{+}}$. Write $\bar{n}=\exp u$, where $u \in \operatorname{Lie}(\bar{N})$. Then we may write $u=\sum_{\alpha \in \Sigma^{+}} u_{\alpha} X_{-\alpha}$ where the $X_{-\alpha}$ are the bases for the root spaces, and $u_{\alpha} \in \mathbb{R}$. (For notational simplicity, if the multiplicity $m_{\alpha}$ of $\alpha$ is greater than 1 , we think of $\alpha$ as repeated $m_{\alpha}$ times in $\Sigma)$. Then $a^{-1} \bar{n} a=\exp \left(\sum_{\alpha \in \Sigma^{+}} u_{\alpha} e^{\alpha(a)} X_{-\alpha}\right)$.

By [EF, Lemmas A.2 and A.3] (or by [Mo, Lemma 7.6]), for $u \in \operatorname{Lie}(\bar{N})$, if either $\|u\| \gg 1$ or $d(\pi(\exp u), \pi(A)) \gg 1, d(\pi(\exp u), \pi(A))$ is comparable to $\log \|u\|$, where $\|\cdot\|$ is the Euclidean norm on $\operatorname{Lie}(\bar{N})$. In our case $\|u\| \gg 1$ since $r \gg 1$. Hence

$$
\lambda_{1} \log \left(\sum_{\alpha \in \Sigma^{+}} u_{\alpha}^{2} e^{2 \alpha(a)}\right) \leq d\left(e, \pi\left(a^{-1} \bar{n} a\right)\right) \leq \lambda_{2} \log \left(\sum_{\alpha \in \Sigma^{+}} u_{\alpha}^{2} e^{2 \alpha(a)}\right) .
$$

We write $a=\exp x$ for $x \in \operatorname{Lie}(A)=\mathbb{R}^{n}$. Then by definition (see [EF, §2]), $\alpha(a)=\alpha(x)$, and the function $\alpha$ on $\mathbb{R}^{n}$ is linear. Then

$$
\max _{\alpha \in \Sigma^{+}} \lambda_{1} \log \left(u_{\alpha}^{2} e^{\alpha(2 x)}\right) \leq d\left(e, \pi\left(a^{-1} \bar{n} a\right)\right) \leq \max _{\alpha \in \Sigma^{+}} \lambda_{2} \log \left(C u_{\alpha}^{2} e^{\alpha(2 x)}\right) .
$$

Exponentiating this, and using (58) we get:

$$
\max _{\alpha \in \Sigma^{+}} u_{\alpha}^{2 \lambda_{1}} e^{\alpha\left(2 x \lambda_{1}\right)-\delta} \leq \exp d\left(F_{2}, \pi(g a)\right) \leq C^{\lambda_{2}} \max _{\alpha \in \Sigma^{+}} u_{\alpha}^{2 \lambda_{2}} e^{\alpha\left(2 x \lambda_{2}\right)+\delta} .
$$

Since $\mathfrak{C} \cap \operatorname{Nbhd}\left(F_{2}, r\right)$ is the set $\left\{g \exp x \in \mathfrak{C} \mid d\left(F_{2}, \pi(g \exp x)\right) \leq r\right\}$, this implies (62).

Pick $r_{0} \gg 1$ (depending only on $X$ ), and let $\mathcal{Q}=\bigcup_{w \in W} \mathcal{P}_{w}\left(r_{0}\right)$. Then, by the structure of $\mathcal{P}_{w}(r), h d\left(F_{1} \cap \operatorname{Nbhd}\left(F_{2}, r\right), \mathcal{Q}\right)=O(r)$. We now show that there exists a convex polyhedron $\mathcal{P}$ such that $h d(\mathcal{P}, \mathcal{Q})=O(r)$. This would imply (i). First suppose $G=S L(n+1, \mathbb{R})$. In view of (61) (changing $w^{\prime \prime}$ to $w$ ) it is enough to show that for some polyhedron $\mathcal{P}$,

$$
h d\left(\left\{\pi(g a) \in F_{1} \quad: \quad d(\pi(g a), \pi(w a)) \leq r\right\}, \mathcal{P}\right)=O(r) .
$$

But,

$$
d(\pi(g a), \pi(w a))=d\left(\pi\left(a^{-1} w^{-1} g a\right), e\right) .
$$

Let $g_{i j}$ denote the entry of $w^{-1} g$ at row $i$ column $j$. Then, by [EF, Lemma A.2], $\lambda^{-1} \max _{i j}\left|\left(a_{i} / a_{j}\right) g_{i j}\right|^{2} \leq d\left(\pi\left(a^{-1} w^{-1} g a\right), e\right) \leq \lambda \max _{i j}\left|\left(a_{i} / a_{j}\right) g_{i j}\right|^{2}$ where $\lambda$ depends only on $X$. But $a_{i} / a_{j}=e^{\alpha(\log a)}$ for some root $\alpha$ of $S L(n+1, \mathbb{R})$. This implies (63) for the $S L(n+1, \mathbb{R})$ case. For the general case, note that we may take a linear representation of $G$, i.e. an embedding $G$ in $S L(m, \mathbb{R})$ for some $m$. Then the result for $S L(m, \mathbb{R})$ implies $(63)$ but a priori $\mathcal{P}$ may be bounded by generalized hyperplanes of the form $\omega(x) \leq c_{\omega}$, where the $\omega$ 's are the weights of the representation. Still, there are finitely many weights, and each $\omega$ is given by an expression of the form $\omega=\sum_{\alpha} c_{\alpha} \alpha$, where the implied constants depend only on $X$. This, together with the fact that $h d(\mathcal{P}, \mathcal{Q})=O(r)$ where $\mathcal{Q}$ is a union of (nongeneralized) polyhedra implies that $\mathcal{P}$ may also be taken to be bounded by nongeneralized hyperplanes. 
To get (iii) we can take $\mathcal{P}_{2}=\psi\left(\mathcal{P}_{1}\right) \cap \mathcal{P}(r)$ where $\mathcal{P}(r)$ is as in part (i) with $F_{1}$ and $F_{2}$ interchanged.

B.2. Angles between Weyl chambers. Let $\Theta(\cdot)$ denote the function defined in [EF, §2.1].

Lemma B.3 (Hyperbolic geometry of $X$ ). There exist constants $\nu, \lambda_{2}$ depending only on $X$ such that the following holds: Suppose $x, y \in X$ satisfy $\alpha(x) \geq r, \alpha(y) \geq$ $r$ for all $\alpha \in \sigma$, where $\sigma \subseteq \Delta$ is nonempty. Then, if $d_{K}\left(\Theta(x), \Theta(y) K_{\sigma}\right) \geq e^{-\nu r}$, $d(x, y) \geq \lambda_{2} r$.

Proof. This is stated and proved in $[\mathrm{EF}]$ as Lemma A.4 in the case where $G=$ $\operatorname{SL}(n, \mathbb{R})$. This case implies the general case by an embedding argument, as in the proof of [EF, Lemma 4.1].

Lemma B.4 (Angles between Weyl chambers). There exist constants $\nu, \nu^{\prime}, \lambda_{2}$ depending only on $X$ such that the following holds: Suppose $k_{1}, k_{2} \in K, \mathfrak{C}_{i}=\pi\left(k_{i} A^{+}\right)$, and there exist points $z_{i} \in \mathfrak{C}_{i}$ such that $d\left(z_{1}, z_{2}\right) \leq \lambda_{2} r$, and $d\left(z_{i}, \partial \mathfrak{C}_{i}\right) \geq \nu^{\prime} r$. Then $d_{K}\left(k_{1}, k_{2}\right) \leq e^{-\nu r}$.

Proof. This follows from Lemma B.3 with $\sigma=\Delta$. The condition that $\alpha\left(z_{i}\right) \geq r$ for all $\alpha \in \Delta$ is implied by $d\left(z_{i}, \partial \mathfrak{C}_{i}\right) \succ r$.

Lemma B.5 (Hyperbolic geometry). Let $\gamma_{1}(t)$ and $\gamma_{2}(t)$ be two geodesic rays, parameterized by arc length, emanating from the point $e$ in a space of $Y$ negative curvature (bounded away from zero). Pick a sufficiently large number $Q$, and let $t$ denote the largest value of $t^{\prime}$ such that $d\left(\gamma_{1}\left(t^{\prime}\right), \gamma_{2}\left(t^{\prime}\right)\right) \leq Q$. Then (for sufficiently large $t$ ),

$$
c_{1} \log (1 / \theta)<t<c_{2} \log (1 / \theta)
$$

where $\theta$ is the angle between $\gamma_{1}$ and $\gamma_{2}$, and $c_{1}$ and $c_{2}$ depend only on $Q$ and $Y$.

Proof. Standard.

Lemma B.5 implies the following partial converse to Lemma B.4:

Lemma B.6. Suppose $\alpha \in \Delta, k_{2} \in k_{1} K_{\alpha}$, and let $\theta=d_{K}\left(k_{1}, k_{2}\right)$. Then for any sufficiently large $Q$ (depending only on $X$ ) there exist $z_{i} \in \pi\left(k_{i} A^{+}\right)$such that $d\left(z_{1}, z_{2}\right) \leq Q, d\left(z_{i}, e\right) \leq \lambda|\log \theta|$ and $d\left(z_{i}, \partial \pi\left(k_{i} A^{+}\right)\right) \geq \nu_{Q}|\log \theta|$. The constant $\nu_{Q}$ depends only on $Q$ and $X$, and the constant $\lambda$ depends only on $X$.

Remark. This lemma holds even without the assumption that $k_{2} \in k_{1} K_{\alpha}$.

Proof. Let $t=t(Q, \theta)$ be as in Lemma B.5, relative to the geodesics $\gamma_{i}(t)=$ $k_{i} \exp \left(t H_{\alpha}\right)$, where $H_{\alpha} \in \mathfrak{a}$ is a unit vector orthogonal to the hyperplane $\alpha=0$. Then $H_{\alpha}$ belongs to the Lie algebra of $M_{\alpha}$, and thus the $\gamma_{i}$ lie in $X_{\alpha}=M_{\alpha} /$ $\left(K \cap M_{\alpha}\right)$. Since $X_{\alpha}$ has rank one, Lemma B.5 applies, and (for $\theta \ll 1$ )

$$
c_{Q}^{\prime \prime}|\log \theta| \leq t \leq c_{Q}^{\prime}|\log \theta|
$$

where $c_{Q}^{\prime}$ and $c_{Q}^{\prime \prime}$ depend only on $Q$ and $X$. Pick a generic positive unit vector $v$ in the Lie algebra of $A_{\alpha}$ (i.e. the hyperplane $\alpha=0$ ), and choose $\nu>0$ such that $v+\nu H_{\alpha} \in \mathfrak{a}^{+}$. Then the points $z_{i}=\exp \left(k_{i} t\left(v+\nu H_{\alpha}\right)\right)$ satisfy the conditions of the lemma. 


\section{B.3. Equivalence of Weyl chambers.}

Lemma B.7 (Containment of chambers). Suppose $\mathfrak{C}_{1}$ and $\mathfrak{C}_{2}$ are Weyl chambers, $L$ is a $\sigma$-wall, and suppose that outside a sufficiently large ball, $L \subset \mathfrak{C}_{1}[\lambda \epsilon] \cap \mathfrak{C}_{2}[\lambda \epsilon]$. Let $k_{1}, k_{2} \in K / M$ be such that $\mathfrak{D}_{i}=\pi\left(k_{i} A^{+}\right)$is equivalent to $\mathfrak{C}_{i}$. Then $k_{2} \in k_{1} K_{\sigma}$, and $\mathfrak{D}_{1}$ and $\mathfrak{D}_{2}$ share a $\sigma$-wall. In particular, if $\mathfrak{C}_{2} \subset \mathfrak{C}_{1}[\lambda \epsilon]$, then $k_{1}=k_{2}$.

Proof. After replacing the $\mathfrak{C}_{i}$ by equivalent Weyl chambers, we may assume that $\mathfrak{C}_{i}=\pi\left(k_{i} A^{+}\right)$. Also, after replacing $L$ by a wall passing through $e$ and a finite Hausdorff distance away, we may assume $L=\pi\left(k A_{\sigma}^{+}\right)$for some $k \in K$. For any $r>0$ there exists $a_{r} \in \overline{A^{+}}$such that $\alpha\left(a_{r}\right)=r$ for $\alpha \in \sigma, \alpha\left(a_{r}\right)=0$ for $\alpha \notin \sigma$. Then $\pi\left(k a_{r}\right) \in L$. Since $L \subset \pi\left(k_{i} A^{+}\right)[\lambda \epsilon]$, there exist $a_{i} \in A^{+}$such that $d\left(\pi\left(k_{i} a_{i}\right), \pi\left(k a_{r}\right)\right)=O(\epsilon r)$. By [EF, Lemma 3.1], $d(x, y) \succ|\alpha(x)-\alpha(y)|$ for all $x, y \in X, \alpha \in \Delta$. Hence we may assume $\alpha\left(a_{i}\right) \geq r / 2$ for all $\alpha \in \sigma$. By Lemma B.3 if $k_{i} \notin k K_{\sigma}$, for sufficiently large $r, d\left(\pi\left(k_{i} a_{i}\right), \pi\left(k a_{r}\right)\right) \succ r$. This is a contradiction.

The following lemma is well known.

Lemma B.8 (Equivalent chambers). Let $\mathfrak{C}$ be a Weyl chamber in $X$ based at $p$. Then there exists an equivalent Weyl chamber $\mathfrak{C}^{\prime}$ based at e such that $h d\left(\mathfrak{C}, \mathfrak{C}^{\prime}\right)=$ $O(d(p, e))$.

Proof. We may assume $\mathfrak{C}=\pi\left(g A^{+}\right)$for $g \in G$. Write $g=k a n$ using the Iwasawa decomposition. Let $\mathfrak{C}^{\prime}=\pi\left(k A^{+}\right)$. Then, for $a_{1} \in A^{+}$,

$$
\begin{aligned}
d\left(\pi\left(g a_{1}\right), \pi\left(k a_{1}\right)\right) & =d\left(\pi\left(k a_{1}\left(a_{1}^{-1} a n a_{1}\right)\right), \pi\left(k a_{1}\right)\right) \\
& =d\left(\pi\left(a_{1}^{-1} a n a_{1}\right), e\right) \prec d(\pi(a n), e)=d(g e, e) .
\end{aligned}
$$

Thus, $h d\left(\mathfrak{C}, \mathfrak{C}^{\prime}\right)=O(d(g e, e))$.

\section{B.4. Convex polyhedra in $\mathbb{R}^{n}$.}

Lemma B.9 (Convex polyhedra). Let $P$ be a (possibly unbounded) convex polyhedron in $\mathbb{R}^{n}$. For every hyperplane $L$ of $\partial P$, let $d_{L}$ denote the maximum distance between a point of $P$ and $L$. Let $d_{P}$ be the smallest of $d_{L}$. Then the injectivity radius $R_{P}$ of $P$ satisfies

$$
R_{P} \geq \frac{1}{n+1} d_{P}
$$

Proof. Let $B(c, R)$ be a ball of maximal radius $R$ inscribed in $P$. Suppose that there are exactly $n+1$ hyperplanes $L_{k}, k=1, \ldots, n+1$, of $\partial P$ that are tangent to $B(c, R)$ and form a simplex $Q$. Let $d_{k}, k=1, \ldots, n+1$, be the distance between $L_{k}$ and the opposite vertex of $Q$ and let $d_{Q}$ be the smallest of $d_{k}$. Since $Q \supset P$ we have $d_{Q} \geq d_{P}$. We claim that $R \geq \frac{1}{n+1} d_{Q}$. Indeed, connecting $c$ with all vertices of $Q$ we get $n+1$ pyramids. The volume of the $k$-th pyramid is $\frac{1}{n} R A_{k}$ where $A_{k}$ is the area of $Q \cap L_{k}$. Therefore for the volume of $Q$ we get $V_{Q}=\frac{1}{n} R \sum_{k=1}^{n+1} A_{k}$. Also we have $V_{Q}=\frac{1}{n} d_{k} A_{k}, k=1, \ldots, n+1$. Therefore $V_{Q}=\frac{1}{n+1} \sum_{k=1}^{n+1} \frac{1}{n} d_{k} A_{k} \geq$ $\frac{1}{n(n+1)} d_{Q} \sum_{k=1}^{n+1} A_{k}$. Hence $R \geq \frac{1}{n+1} d_{Q} \geq \frac{1}{n+1} d_{P}$.

In the general case if the number of hyperplanes $L$ of $\partial P$ tangent to $B(c, R)$ is $\geq(n+1)$ we can achieve, by slightly perturbing these hyperplanes (and hence changing $P$ ), that exactly $n+1$ hyperplanes are tangent to $B(c, R)$ and they form a simplex. After getting the inequality $R \geq \frac{1}{n+1} d_{P}$ for the new polyhedron we roll 
back all changed hyperplanes and get by taking the limit that the inequality holds for the original polyhedron $P$ too.

If the number of hyperplanes in $P$ tangent to $B(c, R)$ is less than $n+1$, then there must be a subspace $S$ in $\mathbb{R}^{n}$ of some dimension $m \geq 1$ that is parallel to all such hyperplanes. Then in the orthogonal complement to $S$ we repeat the estimates above and get $R \geq \frac{1}{n-m+1} d_{P}$.

\section{ACKNOWLEDGEMENTS}

Thanks to Bob Zimmer for his support and encouragement, to Benson Farb with whom I collaborated on parts of this project, to Richard Schwartz for several useful conversations and to Nimish Shah for supplying the proof of Lemma 8.1.

\section{REFERENCES}

[B] K. Brown, Buildings, Springer-Verlag, New York, 1989. MR 90e:20001

[CC] J. Cannon and D. Cooper, A characterization of cocompact hyperbolic and finite-volume hyperbolic groups in dimension three, Trans. AMS 330 (1992), 419-431. MR 92f:22017

[Dru] C. Drutu, Quasi-isometric classification of semisimple groups in higher rank, Preprint.

[EF] A. Eskin and B. Farb, Quasi-flats and rigidity in higher rank symmetric spaces, Journal Amer. Math. Soc. Vol 10, No. 3, 1997, pp. 653-692. CMP 97:11

[Fa] B. Farb, The quasi-isometry classification of lattices in semisimple Lie groups, Math. Res. Lett. Vol. 4, No. 5 (1997), pp. 705-717.

[FM] B. Farb and L. Mosher, A rigidity theorem for the solvable Baumslag-Solitar groups (with an appendix by D. Cooper), to appear in Inventiones Math.

[FS] B. Farb and R. Schwartz, The large-scale geometry of Hilbert modular groups, J. Diff. Geom. 44, No.3 (1996), pp 435-478. CMP 97:07

[KL] B. Kleiner and B. Leeb, Rigidity of quasi-isometries for symmetric spaces of higher rank, to appear in Publ. Math. IHES.

[LMR] A. Lubotzky, S. Mozes, M. S. Raghunathan, The Word and Riemannian Metrics on Lattices of Semisimple Groups, preprint.

[Mo] G.D. Mostow, Strong Rigidity of Locally Symmetric Spaces, Annals of Math. Studies, No. 78, Princeton Univ. Press, 1973. MR 52:5874

[Mu J.R. Munkries, Elements of Algebraic Topology, Benjamin/Cummings Publishing Company, Menlo Park, 1984.

[Pa] P. Pansu, Metriques de Carnot-Caratheodory et quasiisometries des espaces symmetriques de rang un, Annals of Math. 129 (1989), p.1-60. MR 90e:53058

[S1] R. Schwartz, The Quasi-Isometry Classification of Rank One Lattices, IHES Sci. Publ. Math., vol. 82, (1996). MR 97c:22014

[S2] R. Schwartz, Quasi-Isometric Rigidity and Diophantine Approximation, Acta Math. 177 (1996), No. 1, pp. 75-112. MR 97m:53093

[Sh] N. Shah, Invariant measures and orbit closures on homogeneous spaces for actions of subgroups generated by unipotent elements, To appear in the Proceedings of the International Colloquium on Lie Groups and Ergodic Theory, TIFR, Bombay, 1996.

[Ti] J. Tits, Buildings of spherical type and finite BN-pairs, Lecture Notes in Math., Vol. 386, Springer-Verlag, 1974. MR 57:9866

[Zim] R. Zimmer, Ergodic Theory and Semisimple Groups, Birkhauser Boston, Inc., 1984. MR 86j:22014

Department of Mathematics, University of Chicago, 5734 S.University Ave, Chicago, ILLINOIS 60637

E-mail address: eskin@math.uchicago.edu 